University of Tennessee Health Science Center UTHSC Digital Commons

$11-2021$

\title{
Role of SMAD2 and SMAD3 on Adipose Tissue Development and Function
}

\author{
Roshan Kumari \\ University of Tennessee Health Science Center
}

Follow this and additional works at: https://dc.uthsc.edu/dissertations

Part of the Medical Cell Biology Commons, and the Medical Genetics Commons

\section{Recommended Citation}

Kumari, Roshan (https://orcid.org/0000-0002-4759-6046), "Role of SMAD2 and SMAD3 on Adipose Tissue Development and Function" (2021). Theses and Dissertations (ETD). Paper 568. http://dx.doi.org/ 10.21007/etd.cghs.2021.0550.

This Dissertation is brought to you for free and open access by the College of Graduate Health Sciences at UTHSC Digital Commons. It has been accepted for inclusion in Theses and Dissertations (ETD) by an authorized administrator of UTHSC Digital Commons. For more information, please contact jwelch30@uthsc.edu. 


\title{
Role of SMAD2 and SMAD3 on Adipose Tissue Development and Function
}

\begin{abstract}
Introduction: Obesity and its associated metabolic syndrome are major medical problems worldwide including United States. Adipose tissue is the primary site of energy storage, playing important roles in health. Adipose tissue also has other critical functions, producing adipocytokines and contributing to normal nutrient metabolism, which in turn play important roles in satiety, inflammation, and total energy homeostasis. Activin A and activin B play important roles in maintaining body composition and energy homeostasis. This dissertation highlights the role of activin/SMADs signaling in adipose tissue development, function, and maintenance.
\end{abstract}

SMAD2/3 proteins are downstream mediators of transforming growth factor- $\beta$ (TGF $\beta$ ) family signaling, including activins, which regulate critical preadipocyte and mature adipocyte functions. Previous studies have demonstrated that Smad2 global knockout mice exhibit embryonic lethality, whereas global loss of Smad3 protects mice against diet-induced obesity and the direct contributions of Smad2 and Smad3 in adipose tissues individually or in combination and the responses of these tissues to activin signaling are unknown. Additionally, our lab demonstrated that the combined loss of activin A and activin B have reduced adiposity in mice and appearance of brown-like cells in visceral white adipose tissue. However, the cell-autonomous role of activins on cell proliferation and differentiation remained unknown in vitro. My hypothesis was that activin signaling regulate adipocyte differentiation and functions via SMAD2/ 3-mediated mechanism(s) and that the individual or combined adipose-specific deletion of SMAD2/ SMAD3 would result in reduced adiposity similar to activin deficient mice.

Objective: Here, we sought to determine the primary effects of adipocyte-selective reduction of Smad2 or Smad3 individually and in combination, on diet-induced adiposity and to establish whether preadipocytes isolated from subcutaneous and visceral white adipose tissues differ in their differentiation capacity. We also assessed the role of activins on cell proliferation and differentiation using an in vitro model.

Research Design: To assess the adipose-selective requirements of Smad2, Smad3 and Smad2/3, we generated three lines of adipose-selective conditional knockout (cKO) mice including Smad2cKO, Smad3cKO, and Smad2/3 double cKO mice using Smad2 and/or Smad3 "floxed" mice intercrossed with Adiponectin-Cre mice. Additionally, we isolated preadipocytes and examined adipogenic activity of visceral and subcutaneous preadipocyte and the effects of activin on preadipocyte proliferation and differentiation in vitro. Furthermore, we used mouse embryonic fibroblasts (MEFs) from wild type mice and activin double knockout mice to study the cell autonomous role of activin on differentiation and cell fate.

Results: Our results demonstrated that subcutaneous preadipocytes differentiate uniformly and almost all wildtype subcutaneous preadipocytes differentiated into mature adipocytes. In contrast, visceral preadipocytes differentiated poorly. Exogenous activin A promoted proliferation and suppressed differentiation of subcutaneous preadipocytes more robustly given that visceral adipocytes differentiate poorly at baseline. Additionally, global knockout of activin A and B promoted differentiation and browning in differentiated MEFs in vitro consistent with in vivo studies. Furthermore, we showed that Smad2cKO mice did not exhibit significant effects on weight gain, irrespective of diet, whereas Smad3cKO male mice displayed a trend of reduced body weight on high fat diet. On both (LFD and HFD) diets, Smad3cKO male mice displayed an adipose depot-selective phenotype, with significant reduction in subcutaneous fat mass but not visceral fat mass. Smad2/3cKO male mice did not show any difference in body weight or fat mass compared to control mice. Female mice with adipose-selective combined deletion of Smad2/3, displayed reduced body weight and reduction of fat mass in both visceral and subcutaneous depot with higher metabolic rate on HFD compared to control littermates. 
Conclusions: Our study demonstrated that Smad3 is an important contributor to the development and/or maintenance of subcutaneous white adipose tissue in a sex-selective fashion. Combined reduction of Smad2/3 protects female mice from diet induced obesity and is important for visceral and subcutaneous depots in a sex-selective fashion. These findings have implications for understanding SMAD-mediated, depot selective regulation of adipocyte growth and differentiation. Activin treatment promoted proliferation of preadipocytes, while activin deficiency promoted differentiation and altered the phenotypic characteristics of White adipocytes to brown-like cells in vitro consistent with in vivo.

\section{Document Type}

Dissertation

Degree Name

Doctor of Philosophy $(\mathrm{PhD})$

\section{Program}

Biomedical Sciences

\section{Research Advisor}

Chester W. Brown, PhD

\section{Keywords}

Adipogenesis, Genetics, Obesity, SMAD2, SMAD3, White adipose tissue

\section{Subject Categories}

Medical Cell Biology | Medical Genetics | Medical Sciences | Medicine and Health Sciences 


\title{
University of Tennessee Health Science Center
}

\author{
DOCTORAL DISSERTATION
}

\section{Role of SMAD2 and SMAD3 on Adipose Tissue Development and Function}

Author:

Roshan Kumari
Advisor:

Chester W. Brown, MD, PhD

Dissertation Presented for The Graduate Studies Council of The University of Tennessee Health Science Center in Partial Fulfillment of Requirements for the Doctor of Philosophy degree from The University of Tennessee

in

Biomedical Sciences: Genetics, Genomics and Informatics College of Graduate Health Sciences 
Chapter 4 (c) 2021 John Wiley \& Sons, Inc. All other material (c) 2021 by Roshan Kumari. All rights reserved.

Modified with permission Masters/Doctoral Thesis LaTeX Template

Version $2.5(8 / 27 / 2017)$ http://www. LaTeXTemplates . com Creative Commons License CC BY-NC-SA 3.0 
This dissertation is dedicated to my respected maternal grandparents, Late Prof. Surendra Prasad Roy and Late Gayatri Devi for their unconditional father's and mother's love, encouragement, inspiration, never-ending support for my personal and career growth in every second of my life and with whom I have grown up, and without whom it would not have been possible to achieve my dream. 


\section{Acknowledgements}

I would like to express my gratitude and sincere thanks to all the people that have helped me in getting this dissertation work done.

First, I am grateful to my mentor Dr. Chester W. Brown for taking the time to train me, finding a potential in me, giving me an opportunity to complete my training and providing me a right direction in his laboratory. Dr. Brown is a great mentor, and I appreciate his style of training and challenging to think critically about experiments/research article etc. I was constantly inspired by his rationale relating to the context of my research project and current progress in the field of adiposity. This is the most important key to become successful as a graduate student and a step forward in my career that motivated me to think critically. I would also like to thank my mentor for establishing a positive collaborative environment outside the lab and giving me freedom to explore and collaborate as well and thinking about my professional development constantly. Also, I am grateful to him for providing me exceptional personal support in my toughest time of my life. Furthermore, I am also thankful to him who has gone above and beyond to arrange my stipend and thinking about me in the middle of the training so that I can complete my dissertation on time. Overall, I thank him for all the guidance and support during my training at University of Tennessee Health Science Center (UTHSC).

I am also grateful to my dissertation committee members, Dr. Joseph F. Pierre, Dr. Michelle A. Puchowicz, Dr. Athena S. Davenport and Dr. Joan C. Han for their valuable time, guidance, and helpful advice during my training. Also, I am thankful to Dr. Joseph F. Pierre who has gone above and beyond providing me exceptional support for arranging my stipend for year 2021, co-mentorship, and valuable suggestions for grant writing, helping with parental nutrition project, book chapter, techniques and manuscript formulations. I am also thankful to Dr. Michelle A. Puchowicz for her open-door policy and giving me helpful guidance and advice for experiments, metabolic core facility support, manuscript formulations and grant writing whenever needed. Exceptional guidance from my advisor and committee members have been an immense help for my success.

I would like to thank my colleague Dr. Maria Johnson Irudayam for exceptional never-ending support during my research training, and his valuable contributions during my research including teaching different techniques and research discussions. I also thank Monica Dunbar, and past members for all the assistance in Dr. Brown lab. I thank Qusai Al Abdallah and Tahliyah S. Mims for all the help and encouragement from Dr. Pierre lab. I am 
also thankful to Dr. Daniel Johnson from MRC for his valuable time and encouragement. I am also thankful to Dr. Donald B. Thomason, Dr. Donkor, Dr. Byron C. Jones, Dr. Athena S. Davenport, my fellow classmates Jeremiah Afolabi and Dewan Shrestha, and all the tutors for all their support during my education at UTHSC.

I am thankful to the department of Genetics, Genomics, and Informatics for providing me the best student award for the year of 2020. I would like to express my heartfelt appreciation and special thanks to Center for Pediatric Experimental Therapeutics (CPET) Center for fellowship award for 2021. I would also like to thank administrative staff of our college (CGHS), Mr. Larry L. Tague, Ms. Shirley Hancock, Ms. Elizabeth Webb, Ms. Felicia Washington, Ms. Jeddie Maxwell, and Laboratory Animal Care Unit staffs for continuous support from the beginning to the end of my training.

At last, I express my sincere gratitude to my maternal grandfather, Late Prof. Surendra Prasad Roy, and maternal grandmother, Late Gayatri Devi, mother Manju Roy, maternal uncle Sunil Kumar Roy, maternal aunt Savita Roy, sister Kiran Kumari and my beloved husband Dr. Ram P. Kumar, my twin daughters Aditi Roy, Aakanksha Roy (to help me in generating BioRender graphs for this dissertation), son Atharv Kumar, and my friends for their continuous support, encouragement, unconditional love which have been my strength of my achievements. Many thanks to the Lord for everything that I received so far and the future that he planned for me. It was a wonderful journey and I totally enjoyed being a UTHSC students. 


\begin{abstract}
Roshan Kumari

Role of SMAD2 and SMAD3 on Adipose Tissue Development and Function
\end{abstract}

Introduction: Obesity and its associated metabolic syndrome are major medical problems worldwide including United States. Adipose tissue is the primary site of energy storage, playing important roles in health. Adipose tissue also has other critical functions, producing adipocytokines and contributing to normal nutrient metabolism, which in turn play important roles in satiety, inflammation, and total energy homeostasis. Activin A and activin $\mathrm{B}$ play important roles in maintaining body composition and energy homeostasis. This dissertation highlights the role of activin/SMADs signaling in adipose tissue development, function, and maintenance.

SMAD2/3 proteins are downstream mediators of transforming growth factor- $\beta$ (TGF$\beta$ ) family signaling, including activins, which regulate critical preadipocyte and mature adipocyte functions. Previous studies have demonstrated that Smad2 global knockout mice exhibit embryonic lethality, whereas global loss of Smad3 protects mice against diet-induced obesity and the direct contributions of Smad2 and Smad3 in adipose tissues individually or in combination and the responses of these tissues to activin signaling are unknown. Additionally, our lab demonstrated that the combined loss of activin A and activin B have reduced adiposity in mice and appearance of brown-like cells in visceral white adipose tissue. However, the cell-autonomous role of activins on cell proliferation and differentiation remained unknown in vitro. My hypothesis was that activin signaling regulate adipocyte differentiation and functions via SMAD2/3-mediated mechanism(s) and that the individual or combined adipose-specific deletion of SMAD2/SMAD3 would result in reduced adiposity similar to activin deficient mice.

Objective: Here, we sought to determine the primary effects of adipocyte-selective reduction of Smad2 or Smad3 individually and in combination, on diet-induced adiposity and to establish whether preadipocytes isolated from subcutaneous and visceral white adipose tissues differ in their differentiation capacity. We also assessed the role of activins on cell proliferation and differentiation using an in vitro model. 
Research Design: To assess the adipose-selective requirements of Smad2, Smad3 and Smad2/3, we generated three lines of adipose-selective conditional knockout (cKO) mice including Smad2cKO, Smad3cKO, and Smad2/3 double cKO mice using Smad2 and/or Smad3 "floxed" mice intercrossed with Adiponectin-Cre mice. Additionally, we isolated preadipocytes and examined adipogenic activity of visceral and subcutaneous preadipocyte and the effects of activin on preadipocyte proliferation and differentiation in vitro. Furthermore, we used mouse embryonic fibroblasts (MEFs) from wild type mice and activin double knockout mice to study the cell autonomous role of activin on differentiation and cell fate.

Results: Our results demonstrated that subcutaneous preadipocytes differentiate uniformly and almost all wildtype subcutaneous preadipocytes differentiated into mature adipocytes. In contrast, visceral preadipocytes differentiated poorly. Exogenous activin A promoted proliferation and suppressed differentiation of subcutaneous preadipocytes more robustly given that visceral adipocytes differentiate poorly at baseline. Additionally, global knockout of activin A and B promoted differentiation and browning in differentiated MEFs in vitro consistent with in vivo studies. Furthermore, we showed that Smad2cKO mice did not exhibit significant effects on weight gain, irrespective of diet, whereas Smad3cKO male mice displayed a trend of reduced body weight on high fat diet. On both (LFD and HFD) diets, Smad3cKO male mice displayed an adipose depot-selective phenotype, with significant reduction in subcutaneous fat mass but not visceral fat mass. Smad2/3cKO male mice did not show any difference in body weight or fat mass compared to control mice. Female mice with adipose-selective combined deletion of $S m a d 2 / 3$, displayed reduced body weight and reduction of fat mass in both visceral and subcutaneous depot with higher metabolic rate on HFD compared to control littermates.

Conclusions: Our study demonstrated that Smad3 is an important contributor to the development and/or maintenance of subcutaneous white adipose tissue in a sex-selective fashion. Combined reduction of Smad2/3 protects female mice from diet induced obesity and is important for visceral and subcutaneous depots in a sex-selective fashion. These findings have implications for understanding SMAD-mediated, depot selective regulation of adipocyte growth and differentiation. Activin treatment promoted proliferation of preadipocytes, while activin deficiency promoted differentiation and altered the phenotypic characteristics of White adipocytes to brown-like cells in vitro consistent with in vivo. 


\section{Contents}

1 Introduction $\quad 1$

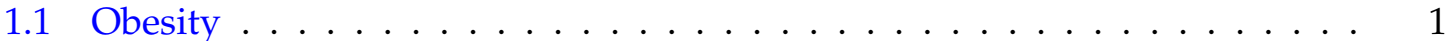

1.2 Scientific Premise . . . . . . . . . . . . . . . . . 3

1.3 Hypothesis and Objectives . . . . . . . . . . . . . . 5

2 Literature Review 9

2.1 An Overview of Obesity . . . . . . . . . . . . . . . . . . . . . 9

2.2 Cause and Consequences of Obesity . . . . . . . . . . . . . 9

2.3 Cell Signaling Crosstalk and Obesity . . . . . . . . . . . . . . . . . 10

2.4 Organs Contributing to Obesity . . . . . . . . . . . . . . . . . . . 10

2.5 Origin and Development of Adipose Tissue and Its Impact on Adiposity . . 11

2.6 White Adipose Tissue (WAT) . . . . . . . . . . . . . . . . . . 12

2.7 Visceral and Subcutaneous WAT . . . . . . . . . . . . . . . . . . . 12

2.8 Brown Adipose Tissue (BAT) . . . . . . . . . . . . . . . . . . . . . . . . . . . . . . . . 13

2.9 Brite Adipose Tissue . . . . . . . . . . . . . . . . . . . . . . . 13

2.10 Roles of Mitochondria in Adipose Tissues and Metabolism . . . . . . . . . . 14

2.11 Adipocyte Hypertrophy and Hyperplasia . . . . . . . . . . . . . . . . . . 14

2.12 Adipose Tissue Macrophages (ATMs) . . . . . . . . . . . . . . . . . . . . . . 15

2.13 An Overview of Activin Signaling . . . . . . . . . . . . . . . . . . . . 15

2.14 Adipogenesis and Key Transcription Factors Regulating Adipogenesis . . . 16

2.15 Activin Signaling and Transcription Factors (SMAD2 and SMAD3) Regulate

Adipogenesis . . . . . . . . . . . . . . . . . 19

2.16 Structure and Function of SMAD2 and SMAD3 . . . . . . . . . . . . . . . 19

2.17 SMAD2 and SMAD3 in Development and Disease . . . . . . . . . . . . . . 23

2.18 Role of Microbiota on Obesity . . . . . . . . . . . . . . . . . . . 24

3 Material and Methods $\quad 27$

3.1 In Vivo Assay . . . . . . . . . . . . . . . . . . . . . . . . . . . . 27

3.1 .1 Generation of Mice . . . . . . . . . . . . . . . . . . . 27

3.1.2 Glucose and Insulin Tolerance Tests . . . . . . . . . . . . . . . 27

3.1.3 Body Composition Measurement . . . . . . . . . . . . . . . . . . 28

3.1.4 Indirect Calorimetry (Comprehensive Laboratory Animal Monitoring

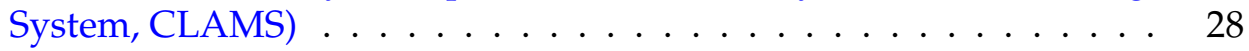


3.1 .5 DNA Isolation and Genotyping . . . . . . . . . . . . . . . . . . . . . . 28

3.1 .6 Tissue Collection . . . . . . . . . . . . . . . . . . . . . . . . . 29

3.1 .7 Histology . . . . . . . . . . . . . . . . . . . . . . . . . . . 29

3.1.8 RNA Isolation and Gene Expression Analysis . . . . . . . . . . . . . 29

3.1 .9 Western Blot Analysis . . . . . . . . . . . . . . . . . 30

3.2 In vitro Assay . . . . . . . . . . . . . . . . . . . . . 30

3.2.1 Mouse Embryo Fibroblasts (MEFs) Isolation and Cell Culture . . . . 30

3.2.2 Preadipocyte Isolation and Differentiation Assay . . . . . . . . . . . . 31

3.2 .3 Immunofluorescence . . . . . . . . . . . . . . . . . . . . . . 31

3.2 .4 Proliferation Assay . . . . . . . . . . . . . . . . . . . . . . . . 32

3.2 .5 Flow Cytometry . . . . . . . . . . . . . . . . . . . . . 32

3.2 .6 RNA-Seq Library Prep . . . . . . . . . . . . . . . . . . . . 32

3.2.7 RNA-Seq Processing, Sequencing, and Analysis of RNA-Seq Data . 33

3.2 .8 Glycerol Estimation . . . . . . . . . . . . . . . . . . 33

3.2 .9 Microscopy . . . . . . . . . . . . . . . . . . 33

3.3 Statistical Analysis . . . . . . . . . . . . . . . . . 33

4 SMAD2/3 Differentially Regulate Adiposity and the Growth of Subcutaneous White Adipose Tissue $\quad 35$

4.1 Introduction . . . . . . . . . . . . . . . . . . 35

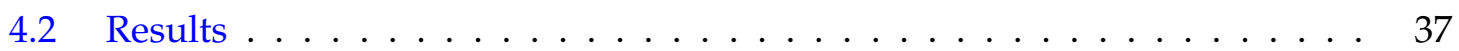

4.2.1 SMAD2 and SMAD3 Proteins Exhibit Different Temporal Expression During Adipose Tissue Development . . . . . . . . . . . . . . . . 37

4.2.2 Activins Regulate Phosphorylation of SMAD2 and SMAD3 in Adipogenic Cells . . . . . . . . . . . . . . . . 37

4.2.3 Subcutaneous and Visceral Preadipocytes Differ in Adipogenic Efficiency Influenced by Activin Signaling . . . . . . . . . . . . . 41

4.2.4 Adipose-Selective Deletion of Smad2 or Smad3 Have Different Effects on Weight Gain Under High-Fat Diet Conditions . . . . . . . . . . . 44

4.2.5 Smad3-Deficient Male Mice Exhibit Differences in Weight Gain and Subcutaneous Fat Mass . . . . . . . . . . . . . . . 46

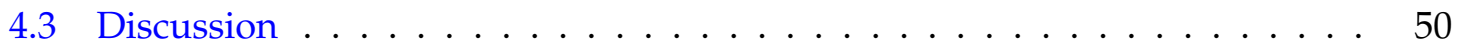

5 Combinatorial Role of SMAD2/3 on Adiposity 57

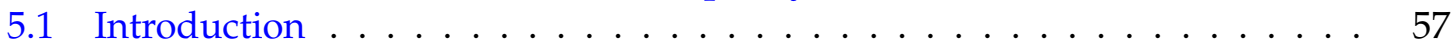

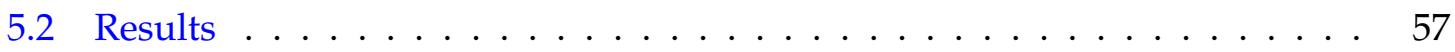

5.2.1 Adipose-Selective Deletion of Smad2/3 Protects Mice from Diet-Induced Obesity in a Sex-Specific Manner . . . . . . . . . . . . . . . 57

5.2.2 Smad2/3-Deficient Female Mice Exhibit Differences in Fat Mass along with Weight Gain . . . . . . . . . . . . . . . . . 59

5.2.3 Smad2/3cKO Mice Show Increased Energy Expenditure . . . . . . . 63 
5.2.4 SMAD2/3 Regulate Key Genes Involved in Adipogenesis, Lipogenesis and Lipolysis . . . . . . . . . . . . . . . . . . . 66

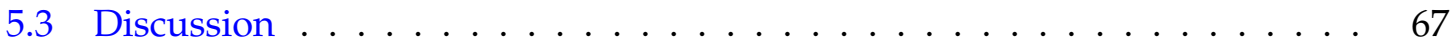

6 Role of Activins on Preadipocyte Proliferation, Differentiation and $\begin{array}{ll}\text { Cell Fate } & 68\end{array}$

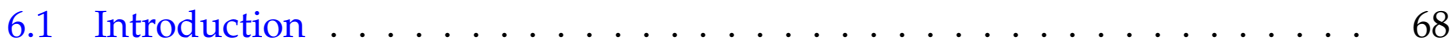

6.2 Results ................................. 69

6.2.1 Activin A Promotes Proliferation of Preadipocytes . . . . . . . . . . . 69

6.2.2 Activin Deficiency Promotes Adipogenesis in Differentiating MEFs 70

6.2.3 Activin Deficiency Induces Browning in Differentiating Activin DKO MEFs . . . . . . . . . . . . . . . . . . 72

6.2.4 Enhanced Mitochondrial Gene Expression in Activin-Deficient Differentiated MEFs . . . . . . . . . . . . . . . . . 72

6.3 Discussion . . . . . . . . . . . . . . . . . . . 79

6.3.1 Early Stage of Browning in Activin DKO Adipocytes Is Intrinsic and Cell Autonomous Due to Complete Loss of Activin A and Activin B

7 Conclusion and Significance $\quad 81$

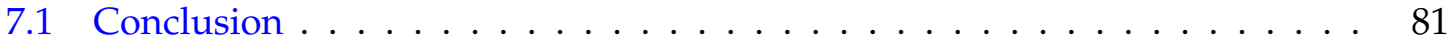

7.2 Future Directions . . . . . . . . . . . . . . . . . 82

$\begin{array}{lr}\text { List of References } & 85\end{array}$

$\begin{array}{ll}\text { Vita } & 105\end{array}$ 


\section{List of Tables}

6.1 KEGG 2019 Mouse Pathways Analysis . . . . . . . . . . . . . . . . . . . . . 77 


\section{List of Figures}

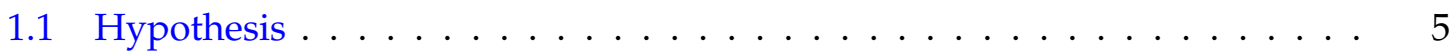

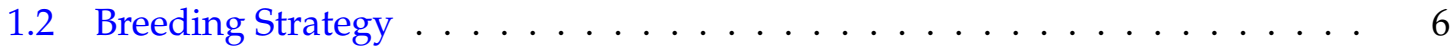

1.3 Mouse Metabolic Phenotyping Flowchart . . . . . . . . . . . . . . . . 7

1.4 Differentiation of Preadipocyte into Mature Adipocytes and Associated Mark-

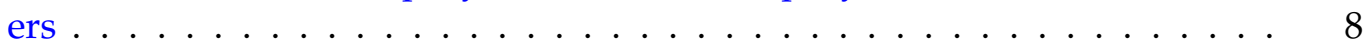

2.1 Adipogensis . . . . . . . . . . . . . . . . . . . . . . . 18

2.2 SMAD-Mediated Activin Signaling Pathway . . . . . . . . . . . . . 20

2.3 Structures of SMAD3, SMAD2, SMAD2 $\beta$ and SMAD4 . . . . . . . . . . . . 22

4.1 SMAD2 and SMAD3 Expression in White Adipose Tissues . . . . . . . . . 38

4.2 SMAD2 and SMAD3 Expression in Differentiating MEF-Derived Adipocytes 39

4.3 Effect of Activin Deficiency on SMAD Expression and Phosphorylation . . . 40

4.4 Activin A, SB431542 and SIS3 Regulate Differentiation of Subcutaneous Preadipocytes ............................ 42

4.5 Activin A, SB431542 and SIS3 Regulate Differentiation of Visceral Preadipocytes 43

4.6 Generation of Smad2cKO and Smad3cKO Mice Using Adiponectin-Cre . . . . 45

4.7 Adipocyte-Selective Deletion of Smad3 in Males Results in Lower Body Weight 47

4.8 Phosphorylation of SMAD3 on LFD or HFD for 8 or 23 weeks . . . . . . . . 48

4.9 Glucose Tolerance Test . . . . . . . . . . . . . . . . . . . . . 48

4.10 Smad2cKO Mice Show Similar Blood Glucose Levels and No Effect on Fat Mass 49

4.11 Adipocyte-Selective Deletion of Smad3 in Males Diminishes Subcutaneous WAT Irrespective of Diet . . . . . . . . . . . . . . . . . . 51

4.12 Smad3cKO Female Show No Effect on Fat Mass . . . . . . . . . . . . . . . 52

4.13 mRNA Levels of Adipogenic Markers in Subcutaneous WAT . . . . . . . . . 52

4.14 Working Model Showing Effects of SMAD2 and SMAD3 on White Adipose

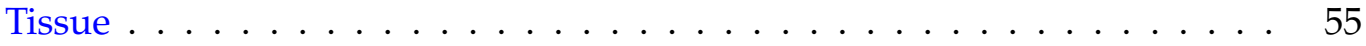

5.1 Generation of Smad2/3cKO Mice Using Adiponectin-Cre . . . . . . . . . . . . . 58

5.2 Lower Body Weight in Smad2/3cKO Mice in Females . . . . . . . . . . . . . . . . 58

5.3 Lower Percent Fat Mass in Smad2/3cKO Mice in Females . . . . . . . . . . . 60

5.4 Adipocyte-Selective Deletion of Smad2/3 in Males Exhibits No Change in Fat

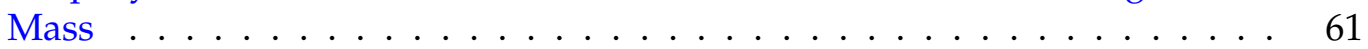

5.5 Diminished Visceral and Subcutaneous WAT in Smad2/3cKO Mice on HFD . 62 
5.6 Indirect Calorimetry Showing Higher Metabolic Rate in Smad2/3cKO Mice on HFD . . . . . . . . . . . . . . . . . . . . . . . . . . . 64

5.7 Indirect Calorimetry Showing Higher Metabolic Rate in Smad2/3cKO Mice on HFD in Fasting State . . . . . . . . . . . . . . . . . . 65

5.8 SMAD2/3 Regulate Key Genes Involved in Adipogenesis, Lipogenesis and

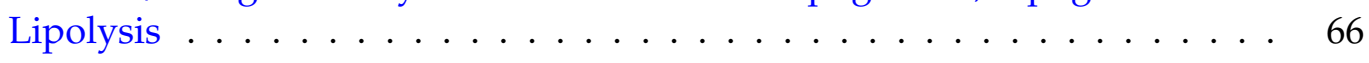

6.1 Activin A Promotes Subcutaneous Preadipocyte Proliferation While Activin Receptor Inhibitor SB431542 Inhibits Proliferation . . . . . . . . . . . . . . . 69

6.2 Apoptosis Assay . . . . . . . . . . . . . . . . . . . . . . 70

6.3 Activin Deficiency Promotes Adipocyte Differentiation of MEFs . . . . . . 71

6.4 Activin Deficiency Induces Browning in MEFs . . . . . . . . . . . . . . . 73

6.5 Enhanced Mitochondrial Gene Expression in Activin Deficient Differentiated MEFs. . . . . . . . . . . . . . . . . . . . . . . 74

6.6 Activin Deficiency Induces Browning Genes in MEFs . . . . . . . . . . . . 75

6.7 Differentially Expressed Genes Share Common Ancestral Lineages . . . . 76

6.8 PI3K-AKT Signaling Pathway. . . . . . . . . . . . . . . . . 78

6.9 Validation of Key Transcription Factors in Activin DKO and WT MEFs . . . 79 


\section{List of Abbreviations}

$\begin{array}{ll}\text { ALK } & \text { Activin like kinase } \\ \text { ASC } & \text { Adipose stem cells } \\ \text { AT } & \text { Adipose tissue } \\ \text { ATGL } & \text { Adipose triglyceride lipase } \\ \text { AUC } & \text { Area under curve } \\ \text { BAT } & \text { Brown adipose tissue } \\ \text { BMI } & \text { Body mass index } \\ \text { CLAMS } & \text { Comprehensive laboratory animal monitoring system } \\ \text { DAPI } & \text { 4'6-Diamidino-2-phenylindole } \\ \text { DIO } & \text { Diet-induced obesity } \\ \text { DKO/dKO } & \text { Double Knockout } \\ \text { DMSO } & \text { Dimethyl sulphoxide } \\ \text { DNA } & \text { Deoxyribonucleic acid } \\ \text { EchoMRI } & \text { Echo magnetic resonance imaging } \\ \text { ESC } & \text { Embryonic stem cells } \\ \text { ETC } & \text { Electron transport chain } \\ \text { FBS } & \text { Fetal bovine serum } \\ \text { FDR } & \text { False dicovery rate } \\ \text { GTT } & \text { Glucose tolerance test } \\ \text { GWAS } & \text { Genome wide association study } \\ \text { HFD } & \text { High fat diet } \\ \text { HSL } & \text { Hormone sensitive lipase } \\ \text { H\&E } & \text { Hematoxylin and eosin } \\ \text { IACUC } & \text { Institutional animal care and use committee } \\ \text { IBD } & \text { Inflammatory bowel disease } \\ \text { ITT } & \text { Insulin tolerance test } \\ \text { KEGG } & \text { Kyoto Encyclopedia of Genes and Genomes } \\ \text { KO } & \text { knockout } \\ \text { LEP } & \text { Leptin } \\ \text { LFD } & \text { Low fat diet } \\ \text { MEFs } & \text { Mouse embryo fibroblasts } \\ \text { M } & \text { Molar } \\ \text { mRNA } & \text { messanger Ribonucleic acid } \\ \text { MSCs } & \text { Mesenchymal stem cells } \\ & \end{array}$


MTT 3-(4,5-Dimethylthiazol-2-yl)-2,5-diphenyltetrazolium bromide)

NEFA Non essential fatty acid

OXPHOS Oxidative phosphorylation

PBS Phosphate buffer saline

RBC Red blood cell

RER Respiratory exchange ratio

RNA Ribonucleic acid

RT-PCR Real time polymerase chain reaction

ROS Reactive oxygen species

REST Relative expression software tool

Smad3cKO Smad3 conditional knockout mice

Smad2cKO Smad2 conditional knockout mice

Smad2/3dcKO Smad2/3 double conditional knockout mice

SEM

TBS

Standard error of mean

TEE

Tris buffer saline

TGF- $\beta$

TNF

Total energy expenditure

UCP1

WAT

Transforming growth factor- $\beta$

Tissue necrotic factor

Uncoupling protein 1

WT

White adipose tissue

Wild type 


\section{Chapter 1}

\section{Introduction}

\subsection{Obesity}

Obesity is a health epidemic worldwide with tremendous impact on the global economy and health care systems. According to National Health and Nutrition Examination Survey (NHANES), the prevalence of obesity has doubled in the past decade and nearly a third of the world's population is now clinically obese. In the United States alone, current data suggests that obesity rates have tripled in adults, and this is a major contributing cause of death (Karagozian, Derdák, and Baffy, 2014; Hruby and F. B. Hu, 2015; Chooi, Ding, and Magkos, 2019). The occurrence of obesity is also not uncommon in children and adolescents. Currently, about $42.4 \%$ of U.S. adults and about $18.4 \%$ of children and adolescents are obese in United States (Matta et al., 2018; Sanyaolu et al., 2019) and data from the CDC shows that US spends 14\% (327 billion) of total health care expenditures annually to treat obesity or obesity-related metabolic disease.

Obesity occurs due to energy imbalance between calories taken in and calories burnt. This imbalance can happen when there is an imbalance in dietary intake such as higher intake of fatty food, due to sedentary lifestyle or genetic defects (Wright and Aronne, 2012). Obesity is a complex disease associated with the development of an array of metabolic diseases including insulin resistance, type 2 diabetes, hypertension, cardiovascular disease, fatty liver disease, and certain types of cancer, collectively termed, the metabolic syndrome (Grundy, 2004; Marchesini et al., 2008; DeFronzo, 2010). Obesity and associated metabolic diseases remain a major problem and lack of effective treatment and the substantial morbidity associated with this illness. Although obesity research has a major impact in recent decades and several anti-obesity medications have been developed, unwanted side effects, including depression, anxiety and other psychiatric symptoms and more research is needed to find effective ways to mitigate the adverse effects of currently available treatments. Therefore, the need is urgent for interventions that prevent the development of obesity or reduce its severity while minimizing the side effects. Investigation of molecular mechanisms that underly multifactorial metabolic abnormalities associated with obesity such as insulin resistance and type 2 diabetes are of great importance. 
Obesity occurs due to an unhealthy increase in white adipose tissue (WAT) (P. Wang et al., 2008; Jo et al., 2009). Adipose tissue plays a crucial role in maintaining energy homeostasis. Unhealthy expansion of WAT dysregulates energy homeostasis by affecting production of adipocytokines such as Leptin, Adiponectin and Resistin (Gardan, Gondret, and Louveau, 2006; Rabe et al., 2008; Bederman et al., 2018). Dysregulation of adipocytokine production and release impacts satiety and is a major determinant of obesity (Unger et al., 2010). Adipose tissue dysfunction occurs with unhealthy enlargement of adipocytes termed, adipocyte hypertrophy, and is associated with infiltration of macrophages within WAT, inflammation and insulin resistance (J. M. Rutkowski, J. H. Stern, and P. E. Scherer, 2015; Choe et al., 2016; Longo et al., 2019). Altered metabolism is in-part driven by changes in the adipose tissue microenvironment (Choe et al., 2016). The enlargement of adipocytes and enhanced adipogenesis participate in the progression to an obese phenotype and altered metabolism (Choe et al., 2016; Longo et al., 2019).

Visceral and subcutaneous WAT depots are well-described for their important roles in obesity and its co-morbidities (Gesta, Y. H. Tseng, and Kahn, 2007). Many researchers agree that visceral WAT and subcutaneous WAT differ anatomically and have different physiological and metabolic functions. For example, visceral white adipose tissue is associated with increased metabolic risk such as insulin resistance, type2 diabetes and inflammation (Y.-W. Wang et al., 2017). In contrast, expansion in subcutaneous white adipose tissue ameliorates insulin resistance and reduces the risk of type 2 diabetes (Mittal, 2019).

Transforming growth factor- $\beta$ (TGF- $\beta$ ) and several other ligands in this family, including activin $A$ and activin $B$, have direct effects on growth and development of a variety of tissues, including adipose, thereby impacting energy homeostasis (C. W. Brown, HoustonHawkins, et al., 2000; Choy, Skillington, and Derynck, 2000; L. Li et al., 2009; C. K. Tan, Leuenberger, et al., 2011; Yadav, Quijano, et al., 2011; Bonomi et al., 2012; M. J. Lee, 2018). The functions of activin/SMADs signaling are also well studied in many tissues, including adipose (Kunihiro Tsuchida et al., 2009; Lonardo et al., 2011; Dani, 2013; Latres et al., 2017; Song et al., 2017). Activins A and B have pleiotropic effects, including preadipocyte proliferation, differentiation, and determining cell fate (C. W. Brown, L. Li, et al., 2003; Hirai et al., 2005; Zaragosi et al., 2010; Zamani and Chester W. Brown, 2011; Namwanje and C. W. Brown, 2016; Xue et al., 2016; Q. Zhu et al., 2018). Activins can signal via SMADmediated pathways and non-SMAD-mediated pathways (p38 MAPK, ERK, JNK). The focus of this study was to investigate the role of activin-SMAD-mediated signaling pathways on adipogenesis in vivo and in vitro.

In SMADs-mediated pathways, activins A and B signal through activin receptor type II (activin receptor IIA (Act RIIA) or activin receptor IIB (Act RIIB) and activin receptors type I (ALK4 and ALK7) and downstream transcriptional transducers, SMAD2 and SMAD3 (K. Tsuchida et al., 2004; Bernard, K. B. Lee, and Santos, 2006). The homo- or heterodimer of SMAD2 and SMAD3 form a complex with the common SMAD, SMAD4. In preadipocytes, SMAD2/SMAD3/SMAD4 regulate the transcription of downstream adipogenic genes with 
additional transcriptional cofactors (Darlington, Ross, and O. A. MacDougald, 1998; C. W. Brown, L. Li, et al., 2003; Hirai et al., 2005; Fei et al., 2010; Zaragosi et al., 2010; Tsurutani et al., 2011; Namwanje and C. W. Brown, 2016; Xue et al., 2016). During adipocyte differentiation, SMAD2/SMAD3 are phosphorylated, thereby inhibiting downstream transcriptional regulators of adipogenesis, $\mathrm{C} / \mathrm{EBP} \alpha, \mathrm{C} / \mathrm{EBP} \beta, \mathrm{C} / \mathrm{EBP} \delta$ and $\mathrm{PPAR} \gamma$ (Darlington, Ross, and O. A. MacDougald, 1998; Choy, Skillington, and Derynck, 2000; Zaragosi et al., 2010; C. K. Tan, Leuenberger, et al., 2011; C. K. Tan, Chong, et al., 2012). Activin A inhibits differentiation via SMAD2 signaling and promotes self-renewal of adipose progenitors by inducing expression of Nanog (Q. Zhu et al., 2018). Although activin A inhibits differentiation in 3T3-L1 pre-adipocytes and in human adipose progenitors (Hirai et al., 2005; Zaragosi et al., 2010; M. J. Lee, 2018), little is known about the role of activins on the differentiation of mouse embryonic fibroblast cells (MEFs) and mouse preadipocytes.

SMAD2 and SMAD3, the downstream mediators of activin signaling, regulate many biological processes during embryogenesis, as well as adipose tissue development, growth, differentiation, and cell fate decisions (Nomura and E. Li, 1998; Weinstein et al., 1998; Datto et al., 1999; C. K. Tan, Leuenberger, et al., 2011; Tsurutani et al., 2011; Yadav, Quijano, et al., 2011). Although there is functional overlap, SMAD2 and SMAD3 also have distinct functions such as the critical role of Smad2 during early gastrulation (Nomura and E. Li, 1998; Waldrip et al., 1998; Weinstein et al., 1998; Heyer et al., 1999; Ju et al., 2006; Kriseman et al., 2019). In contrast, Smad3 global knockout mice are viable and fertile. However, Smad3 knockout mice undergo postnatal growth retardation with immunodeficiencies, predisposition of colorectal cancer and shortened lifespan (Y. Zhu et al., 1998; Ashcroft et al., 1999; Datto et al., 1999; Yang et al., 1999; Q. Li, Pangas, et al., 2008; C. K. Tan, Leuenberger, et al., 2011).

\subsection{Scientific Premise}

The roles of activin A, activin B and SMAD3 on body composition, adipocyte differentiation and energy metabolism have been demonstrated using a variety of activin and Smad3 alleles in vivo (C. W. Brown, L. Li, et al., 2003; C. W. Brown, Houston-Hawkins, et al., 2000; L. Li et al., 2009; Tsurutani et al., 2011; Bonomi et al., 2012; Yadav, Quijano, et al., 2011; C. K. Tan, E. H. Tan, et al., 2013). Global knockout of Smad3 results in reduced adiposity and improved energy metabolism and protects mice from diet induced obesity (C. K. Tan, Leuenberger, et al., 2011; Tsurutani et al., 2011; Yadav, Quijano, et al., 2011; Yadav and Rane, 2012; C. K. Tan, Leuenberger, et al., 2011; Tsurutani et al., 2011; Yadav, Quijano, et al., 2011; C. K. Tan, Chong, et al., 2012; Yadav and Rane, 2012). However, these effects on adipose could be due to contributions from other tissues such as skeletal muscle, liver and pancreas. Despite the attempts to understand the role or activin/SMADs on adiposity, these studies cannot exclude indirect, secondary influences from other metabolically active tissues such as skeletal muscle, pancreas and liver, as shown by the tissue-selective effects of Smad3 deficiency on hepatic gluconeogenesis and pancreatic beta cell apoptosis (Yadav, Devalaraja, 
et al., 2017; J. H. Lee et al., 2020). Overall, the current literature suggests that inactivating activin/SMAD signaling might have beneficial effects on metabolism (Fei et al., 2010; Xue et al., 2016; Tao et al., 2019).

In contrast to Smad3, Smad2 null mice show embryonic lethality due to failures in gastrulation and germ layer patterning (Nomura and E. Li, 1998; Waldrip et al., 1998; Weinstein et al., 1998). Due to embryonic lethality in Smad2 global knockout mice, the role of SMAD2 on adiposity has not been determined. Additionally, the adipose-selective roles of SMAD3 individually and/or the combined adipose tissue-selective knockout of Smad2 and Smad3 have not been assessed in this model. This opens a new platform to address several key questions such as whether adipose selective reduction of Smad2 or Smad3 (adipocyte specific conditional knockout, cKO) has any effect on adiposity and protection from diet-induced obesity. Furthermore, the combinatorial effects of SMAD2 and SMAD3 have not been investigated to understand the selectivity of each SMAD2 and SMAD3 protein and/or cooperation of both proteins in adipocytes. Similar to Smad3 global knockout, whether adipose selective combined reduction of Smad2/3cKO shows beneficial effects on adiposity and metabolism. This conditional double knockout mutant will also inform whether SMAD2 and SMAD3 compensate, cooperate, or antagonize each other in the context of activin signaling.

Our lab made the seminal discovery that activins A and B regulate adiposity and that the combined loss of activin A and activin B reduce adiposity and has beneficial effects on energy metabolism in vivo (C. W. Brown, L. Li, et al., 2003; L. Li et al., 2009). This also transforms the phenotypic characteristics of white adipose tissue (WAT) to brown-like cells, including multilocular smaller adipocyte, UCP1 positive adipocytes and other molecular features and histologic features typically associated with brown adipose tissue (BAT). The molecular mechanisms by which activins regulate energy homeostasis and browning is not well understood. Although SMAD2/3 are the predominant downstream mediators of activin signaling, whether SMAD2 and SMAD3 have overlapping and/or specific functions in adipose tissues in response to activin signaling remains unknown. This leads to an important area of investigation to address whether the adipose selective reduction of SMAD2/3 (a) has beneficial effects on adiposity and (b) improves energy metabolism, including glucose and lipid metabolism and total energy expenditure. Furthermore, in continuation of our previous work in vivo, the role of activins in adipogenesis and cell autonomous effects on cell fate in vitro remain unknown. Additionally, the role of activins on the differentiation of preadipocytes derived from mouse adipose depots remains in question.

Therefore, in my dissertation work, I aimed to investigate the direct effects of downstream mediators of activin signaling on adipose tissue development and function, specifically, adipose-selective effects of Smad2 and Smad3 deficiency individually and in combination of both (Smad2/3cKO) using adipose-specific conditional knockout (cKO) mice. 
Furthermore, I aimed to further characterize the role of activin A on preadipocyte proliferation and differentiation and to examine the difference between two distinct adipose depots using preadipocytes isolated from visceral and subcutaneous WAT. Additionally, I aimed to investigate the cell autonomous roles of activins $\mathrm{A}$ and $\mathrm{B}$ on adipogenesis and cell fate in vitro, using mouse embryonic fibroblast cells (MEFs) isolated from activin A and activin B global knockout mice.

\subsection{Hypothesis and Objectives}

The central hypothesis of my dissertation research was that activin signaling regulates adipocyte differentiation and functions via SMAD2/3-mediated mechanism(s) and that the individual or combined adipose-specific deficiency of SMAD2/SMAD3 would result in reduced adiposity and beneficial changes in energy metabolism (Figure 1.1).

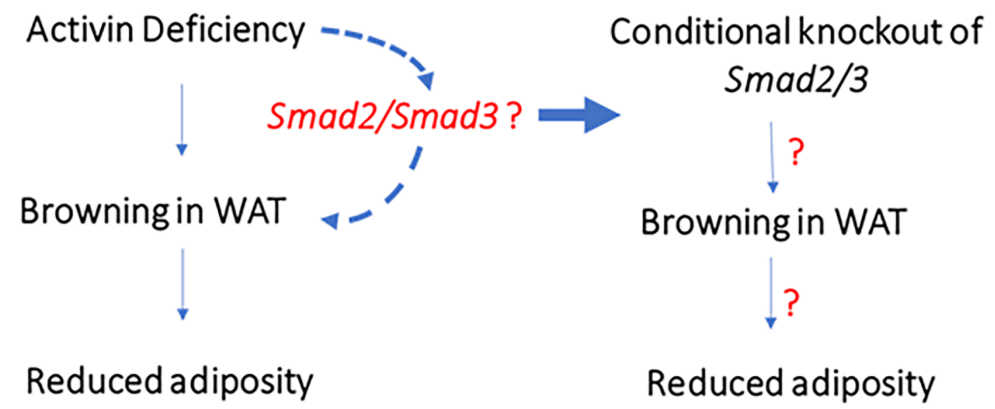

Figure 1.1: Hypothesis. Activin deficiency induces brown-like cells in WAT and reduces adiposity. Activin signaling via SMAD2/3 may contribute to the browning of WAT and reduce fat accumulation.

To test this hypothesis, this research project was comprised of two major studies; the first study included analyses in vivo, and second involved in vitro assays. We tested the hypothesis and achieved the goal of this research project in three specific aims.

Specific Aim 1: The primary objective of this study was to determine the effects of adipose selective deficiency of $S m a d 2, S m a d 3$ and $S m a d 2 / 3$ on adiposity and determine if these mice are resistant to diet-induced obesity. The histological and molecular characteristics associated with adipose selective SMAD2/3 deficiency were also examined.

Rationale: Previous studies have demonstrated that the global knockout of Smad3 in mice protects them from diet-induced obesity (DIO) and improves their overall metabolic profile including glucose tolerance and insulin sensitivity. However, influences due to the global loss of the Smad3 could not be excluded and it is plausible that SMAD3 deficiency in metabolically active organs such as skeletal muscle and liver 
might be contributing to the observed phenotype. Thus, dissecting the tissue specific requirements and the molecular mechanisms by which Smad2 and Smad3 regulate adiposity was the main aim. This study used a mouse model to better understand the role of SMAD2 and SMAD3 on adiposity and DIO and associated molecular mechanisms by which SMAD2/3 plays a crucial role during adipocyte development and function.

To study the function of SMAD2 and SMAD3 signaling cascades in adipose tissue, we had Smad2 flox/flox ,Smad3 ${ }^{\text {flox } / \text { flox }}$ and Adiponectin Cre (Adipoq-Cre) (Ju et al., 2006; Q. Li, Pangas, et al., 2008; Eguchi et al., 2011) mice. Our institutional animal care and use committee has approved all animal studies. I generated three independent lines of adipose selective cKO mice including Smad2cKO (Smad2 flox/flox; Adiponectin $\mathrm{Cre})$, Smad3cKO (Smad3flox/flox; Adiponectin Cre), and Smad2/3cKO (Smad2 flox/flox;

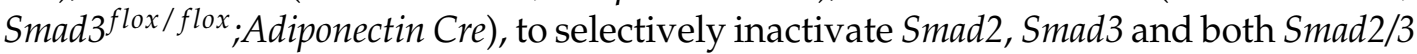
in adipose tissues (Y. Zhu et al., 1998; Ashcroft et al., 1999; Samaras et al., 2010) using Smad2 and Smad3 "floxed" mice intercrossed with Adiponectin-Cre mice (Figure 1.2).

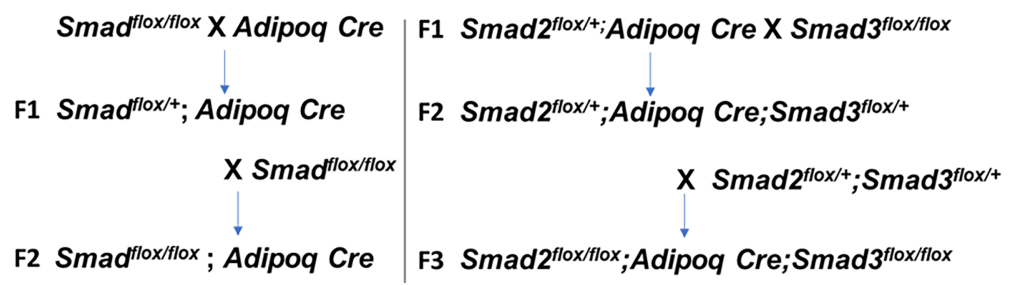

Figure 1.2: Breeding Strategy. Generation of Smad2, Smad3 (denoted as "Smad", left column) and Smad2/3 conditional knockout mice (right column).

These mice were fed a high fat diet (HFD) relative to low fat diet (LFD) and weights were recorded weekly (time course) compared to control mice. These findings helped us to determine whether the adipose selective reduction of Smad2, Smad3 and Smad2/3 have beneficial effects on adiposity. We compared previous findings from global Smad3 knockout mice to the results of this study.

Specific Aim 2: My secondary objective was to determine the impact of individual and combined Smad2/3 deletion on whole body metabolic activity and the metabolic consequences (overall metabolic profile) associated with the adipose-selective reduction of SMAD2, SMAD3 and SMAD2/3.

Rationale: Adipose tissue dysfunction is associated with metabolic changes that occur during obesity including glucose intolerance, insulin resistance, and altered energy homeostasis. Increased fat accumulation, especially visceral fat, has been shown to cause aberrant energy metabolism, leading to insulin resistance. Yadav et al., and Tan et al., (Yadav, Quijano, et al., 2011; C. K. Tan, Leuenberger, et al., 2011) have 
shown that Smad3 global knockout mice have improved glucose tolerance and insulin sensitivity. However, to date there have been no studies using Smad2, Smad3 or both in an adipose selective manner, and additional investigation was needed to explore the adipose selective functions of SMAD2 and SMAD3 in regulating energy homeostasis with nutritionally challenged cKO mice and control mice. We explored the metabolic consequences of $S m a d 2 / 3$ adipose selective deletion. Body fat composition was determined using Echo-MRI. Metabolic profiling was carried out using the Comprehensive Laboratory Animal Monitoring System (CLAMS), glucose-tolerance testing (GTT) and insulin tolerance testing (ITT) (Darrah et al., 2013; Bederman et al., 2018) (Figure 1.3).

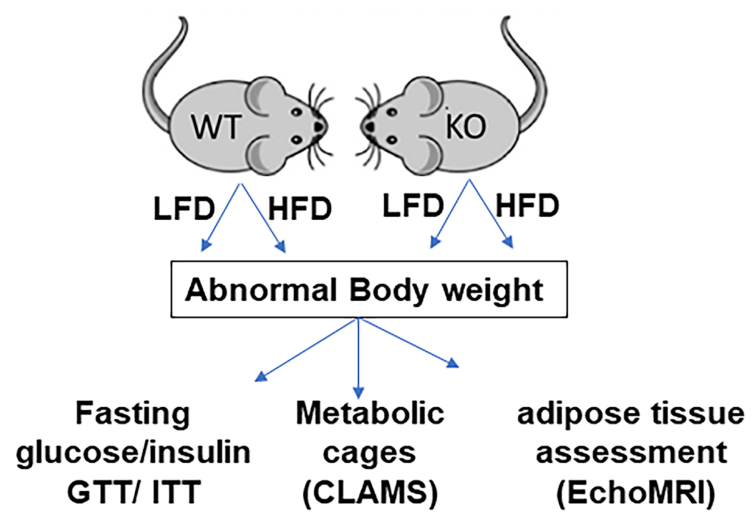

Figure 1.3: Mouse Metabolic Phenotyping Flowchart.

Specific Aim 3: My third objective was to determine the roles of activin and SMADs on cell proliferation, differentiation and energy metabolism using an in vitro model.

3A: My 1st subaim was to determine the cell autonomous effects of activin A/SMAD3 on cell proliferation using preadipocytes.

Rationale: SMAD-mediated activin signaling is known to regulate cell proliferation. Activin A promotes proliferation in 3T3-L1 and adipocyte progenitors (Hirai et al., 2005; Zaragosi et al., 2010). Preadipocytes from visceral and subcutaneous depots were isolated from mouse and proliferation rate was measured by treating them with activin, activin receptor inhibitor, SB431542 and SMAD3 inhibitor, SIS3.

3B: My 2nd subaim was to determine the cell autonomous effects of activin and SMAD3 on adipocyte differentiation and cell fate (browning) using mouse embryonic fibroblast cells or preadipocytes.

Rationale: SMAD-mediated activin signaling is known to regulate adipocyte differentiation. Activin A inhibits differentiation via SMAD2 in 3T3-L1 and human adipocyte progenitors (Hirai et al., 2005; Zaragosi et al., 2010). However, the cell autonomous 
roles of activins on cell differentiation and cell fate in primary culture cells had not been assessed. In this aim, I performed in vitro experiments to understand the roles of activins in adipocyte differentiation and cell fate using mouse embryo fibroblasts (MEFs). Also, several research groups have reported conflicting results regarding the effects of Smad3 on adipocyte differentiation. Yadav et al., (C. K. Tan, Leuenberger, et al., 2011; Yadav, Quijano, et al., 2011) have shown that Smad3 promotes adipogenesis while Tsurtani et al.,(Tsurutani et al., 2011) reported an inhibitory effect. In this study, I examined the cell autonomous effects of Smad3 on preadipocyte differentiation. Differentiated cells were also used to study browning phenomena (Figure 1.4).

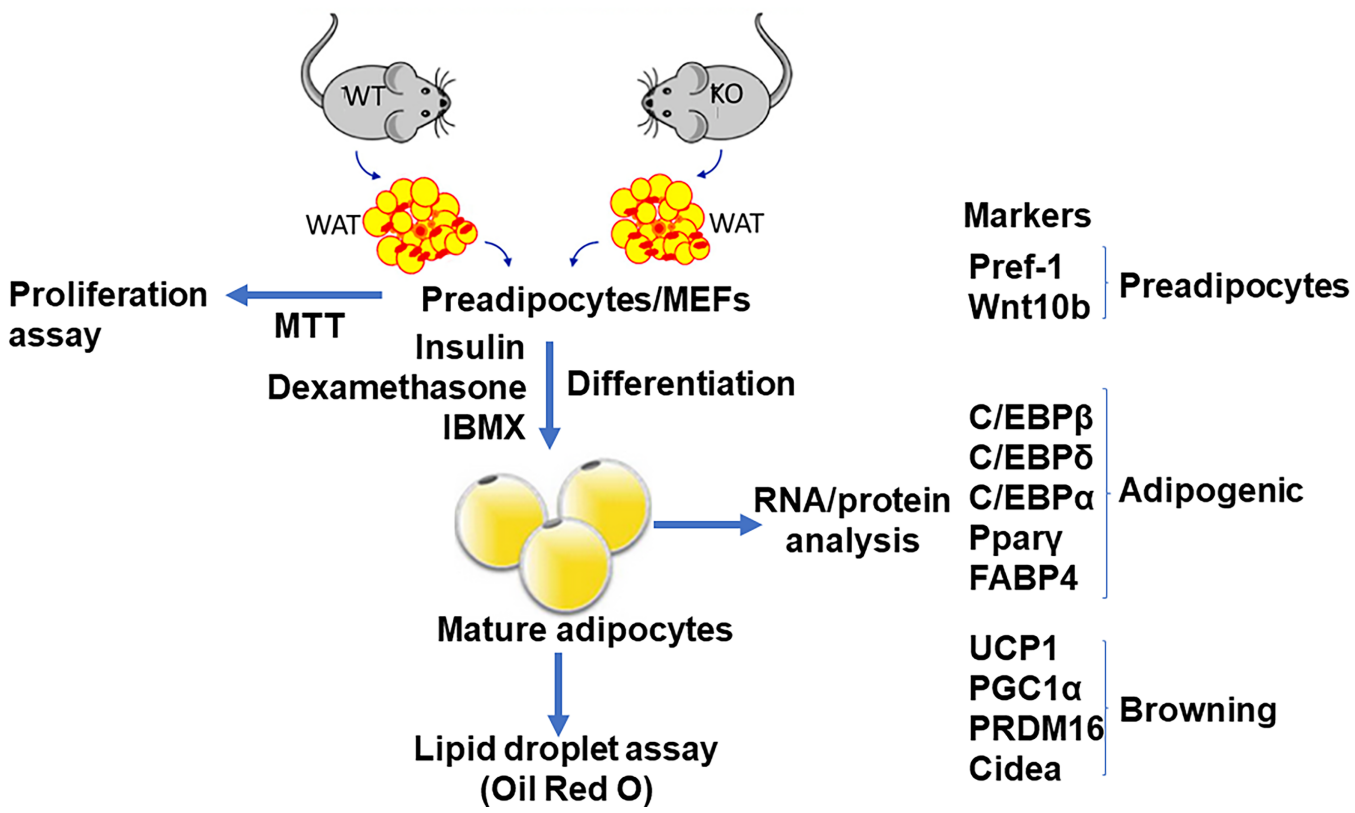

Figure 1.4: Differentiation of Preadipocyte into Mature Adipocytes and Associated Markers. 


\section{Chapter 2}

\section{Literature Review}

\subsection{An Overview of Obesity}

Obesity and its associated metabolic syndrome are major medical problems worldwide. Currently, the obesity rate has reached pandemic proportions rapidly increasing over the past three decades. In the United States alone, the obesity rate exceeds $42 \%$ and is a major risk factor for the development of several chronic diseases such as type 2 diabetes, cardiovascular disease, fatty liver and certain types of cancer (Pi-Sunyer, 2009; Chooi, Ding, and Magkos, 2019). Obesity is associated with increased risk of global morbidity and mortality.

Obesity can be defined based on body mass index (BMI). Adults with BMI between $30-34.9 \mathrm{~kg} / \mathrm{m} 2$ are classified as class I obese, BMI between $35-39.9 \mathrm{~kg} / \mathrm{m} 2$, class II obese and $\mathrm{BMI} \geq 40 \mathrm{~kg} / \mathrm{m} 2$ considered as severe or morbididly obese (Hruby and F. B. Hu, 2015). In children, obesity is classified based on the percentile of BMI depending on the age and sex, considering body composition as body composition differs between male and female children as they grow. Children with BMI $\geq 95$ th percentile are considered obese while BMI $>99$ th percentile is considered severely obese. Also, in the case of athletes and body builders, an increase in BMI does not correlate with poor health status because of increase in muscle mass and misleading BMI associated with weight (Whitlock et al., 2008; Weir and Jan, 2021).

\subsection{Cause and Consequences of Obesity}

While some researchers think that obesity occurs due to social and environmental factors such as diets with high fat content and sedentary lifestyle, others think that genetics plays a predominant role. However, ample evidence in the literature supports a model in which obesity is caused by a combination of genes, nutrient environment, socioeconomic conditions and behavior such as lifestyle choices (Allison et al., 2008). If one considers socioeconomic factors that impact dietary choices, neighborhood food options and affordability are major contributing factors, as food with higher fat content is more affordable than a nutritionally 
well-balanced diet. High caloric density intake and sedentary lifestyle, such as lack of exercise, also contribute to obesity.

\subsection{Cell Signaling Crosstalk and Obesity}

Previous studies have shown that genetics contributes between to $40-70 \%$ to the development of obesity (Herrera and Lindgren, 2010). It can be monogenic or polygenic depending on the individual's obese condition and metabolic outcome. Monogenic obesity is rare and involves single gene defects, most affecting satiety. Among these are Leptin (LEP) or Leptin receptor (LEPR), Melanocortin 4 receptor (MC4R), Proopiomelanocortin (POMC), and Prohormone convertase 1/3 (PCSK1). All have been shown to be sufficient to result in obesity when functionally inactivated (Butler, 2016; Niazi et al., 2019). However, obesity develops due to the combination of several gene-environment interactions, also influenced by the microbiome diversity of an individual (Khera et al., 2019; Hüls et al., 2021). To study polygenic obesity, different approaches have been taken to identify candidate genes, including single nucleotide polymorphisms (SNPs) identified through genome wide association studies (GWAS), and by whole exome or whole genome sequencing of obese individuals. GWAS have identified a total of 445 SNPs and 389 genes that are associated with obesity and metabolism. These genes are involved in many different pathways associated with obesity and related disorders (Cheng et al., 2018). These include key metabolic signaling pathways, AMPK and PI3K-AKT, and genes that impact nutrient metabolism, including FTO, FOXO1, FOXO3, CREB1, PPAR $\gamma$ and IRS1, most of which regulate gene expression as transcriptional co-regulators (Bernhard et al., 2013; Locke et al., 2015). These SNPs regulate disease susceptibility by affecting the miRNA binding such as loci at the binding site of obesity related transcription factors (CEBPs, CREB1), adipogenesis and lipid metabolism (PPAR $\gamma$, ADIPOQ, IRS1, TMEM18, FTO, SMAD7 etc.), and by affecting protein phosphorylation and impacting expression of the adjacent gene (Q. Huang, 2015). The first obesity related gene identified in GWAS is fat mass and obesity associated gene (FTO). The BMI-increasing allele of FTO increases the risk of obesity by 1.2-fold, which is common in different age group and diverse populations (Loos and Yeo, 2014; Merkestein et al., 2015).

\subsection{Organs Contributing to Obesity}

Obesity-related co-morbidities include insulin resistance, type 2 diabetes, fatty liver disease, and dyslipidemia (Hruby and F. B. Hu, 2015; Chooi, Ding, and Magkos, 2019). Obesity can cause problems with many organs including adipose tissue, liver, muscle, arteries, brain, heart, lungs, immune system, pancreas, gallbladder, kidneys, breast, uterus, colon and rectum (Giovannucci et al., 2010). Adipose tissue is the primary site of energy storage and imbalance in energy intake and expenditure can lead to fat deposition in several other organs, leading to insulin resistance and type 2 diabetes. Ectopic fat deposition in the liver will lead to fatty liver disease (hepatic steatosis). Obese people are at high risk for stroke, as 
arteries in the brain can be narrowed by chronic fat deposition, termed, "atherosclerosis", thereby blocking the blood supply to the brain. Arterial damage can also lead to heart attack or peripheral vascular disease. Some risk factors such as insulin resistance and type 2 diabetes can be reversed by maintaining a healthier lifestyle, including increasing physical activity and balanced diet.

\subsection{Origin and Development of Adipose Tissue and Its Impact on Adiposity}

Adipose tissue is loose connective tissue that originates from the differentiation of mesenchymal stem cells (MSCs) into adipocytes during fetal development. Adipose tissue consists of a heterogenous population of cell types such as preadipocytes, adipocytes, fibroblasts, endothelial cells, and various types of immune cells including macrophages, dendritic cells, mast cells, B cells, T cells and neutrophils (Longo et al., 2019; Pyrina et al., 2020). Adipose tissue (AT) is distributed throughout the body in several different depots and is generally classified into two types, WAT and brown adipose tissue (BAT). Both arise from different precursors, and each has distinct architecture, molecular function, mitochondrial physiology and gene expression pattern. In addition to white and brown adipocytes, a third type of adipocyte with "brown-like" characteristics, but inducible appearing in WAT has been well described, termed "brite" or "beige" adipocytes.

The developmental origins of WAT, BAT and brite adipocytes have been rigorously studied and are still controversial. Recent literature suggests that the origins of these adipocytes are more complex than originally hypothesized. According to the literature, mesenchymal stem cells can give rise to Myogenic factor 5- negative precursors (Myf5-) which later assume the lineage of white adipocytes or brite adipocytes. Brite adipocytes emerge in WAT under certain conditions and the emergence of the brown phenotype differs from classical brown adipocytes that originate from Myogenic factor 5 positive (Myf5+) precursors. Mf5+ positive precursors can give rise to classical brown adipocytes and myocytes (Giralt and Villarroya, 2013). Brite adipocytes residing within WAT increase in number after cold exposure, adrenergic stimulation, or administration of thiazolidinediones (TZDs) such a rosiglitazone. These brite adipocytes arise either by differentiation of brite preadipocytes or via trans-differentiation of white adipocytes. However, lineage tracing studies suggest that preadipocytes not only arise from MSCs but also from additional cell types such as vascular endothelial cells, neural crest cells and hematopoietic stem cells that may contribute white and brown adipocyte lineage determination. However, the question still remains as to whether this outcome is due to one cell type or many cell types within WAT. Thus, many different cell types residing in WAT function together and maintain overall whole body energy metabolism (Rosenwald and Wolfrum, 2014). 


\subsection{White Adipose Tissue (WAT)}

WAT is distributed throughout the body. White adipocytes are unilocular with a single, large lipid droplet and fewer mitochondria than brown adipocytes. WAT is distributed in several depots, which include visceral and subcutaneous WAT. Visceral fat is predominantly found around internal organs such as the gonad, kidney, intestine, and pericardium while subcutaneous fat is found beneath the skin. Both visceral and subcutaneous WAT differ in terms of their structure, physiological roles, and molecular and metabolic functions. Notably, dysfunction of visceral fat is associated with components of the metabolic syndrome such as type 2 diabetes and insulin resistance (Frayn, 2000). Subcutaneous WAT serves as a protective cushion and insulates the body from extreme or low temperature . However, the main function of WAT is to store excess energy as lipid, which is regulated by several hormones including insulin, glucagon and catecholamines. Depending on the energy demand, these hormones can work to store or break down triglycerides to release fatty acids and glycerol. Accordingly, WAT regulates lipogenesis, lipolysis, the latter providing the substrate for fatty acid oxidation in the liver. WAT also serves as an endocrine organ by secreting several adipokines such as leptin, adiponectin, resistin, estrogen and cytokines such as TNF-alpha and several interleukins (IL6, ILB, IL10) by a variety of cell types that reside in WAT. These factors regulate a variety of cellular functions in maintaining, satiety/food intake, inflammatory responses, energy substrate metabolism and energy expenditure (Ahima and Lazar, 2008). Leptin and adiponectin have an influence on glucose and lipid metabolism by regulating food intake and insulin sensitivity. Adiponectin increases fatty acid oxidation and insulin sensitivity (B. Lee and J. Shao, 2012; Jennifer H. Stern, Joseph M. Rutkowski, and Philipp E. Scherer, 2016).

\subsection{Visceral and Subcutaneous WAT}

Visceral and subcutaneous WATs differ morphologically, physiologically, and metabolically. They differ in their structure, location, and metabolic function. Visceral fat disproportionately contributes to insulin resistance in obesity due to the accumulation of a variety of immune cells and subsequent production of inflammatory cytokines (Frayn, 2000). The increase in visceral fat mass through hypertrophy is metabolically harmful, increases metabolic risk, overall mortality and can predispose to serious concerns such as type 2 diabetes, insulin resistance, dyslipidemia, heart disease, hypertension, and inflammation, while increase in subcutaneous white adipose tissue through hyperplasia is associated with a more "metabolically healthy" state of improved insulin sensitivity and lowers the risk of type 2 diabetes (Fontana et al., 2007; Hajer, Haeften, and Visseren, 2008). However, it is unclear that increase in fat mass or functional changes in fat tissue contribute to the development of insulin resistance in obese individuals. Although both visceral and subcutaneous WAT express similar sets of genes, their expression levels differ, and when considering differentially expressed genes, mostly lipogenic genes are expressed at higher levels in visceral fat of obese individuals (Samaras et al., 2010; Ronquillo et al., 2019). Additionally, 
transplantation of subcutaneous fat into obese mouse visceral depots resulted in improved metabolic characteristics, including decrease in body weight, and fat pads with improved insulin sensitivity (Tran et al., 2008). Furthermore, the metabolic effect of increased fat mass by transplantation of visceral fat from lean mice resulted in improved insulin sensitivity. This suggests that irrespective of the amount or location of fat, normal metabolic performance of the visceral fat depot is critical to maintain normal metabolic function (Konrad, Rudich, and Schoenle, 2007).

Visceral WAT has larger inflammatory cells than subcutaneous WAT and visceral WAT undergoes lipolysis faster than subcutaneous WAT (Ibrahim, 2010). Additionally, using adipocyte stem cells (ASC) researchers showed that the functional differences between visceral and subcutaneous depots start at the precursor level and remain in mature adipocytes. The depots display distinct proliferation and differentiation rates with subcutaneous ASC having higher proliferation rate, adipogenic efficiency and membrane potential than visceral. The depots start showing functional differences at very early stages of development, so the differences are not restricted to mature adipocytes (Merrick, Sakers et al. 2019). The differences in precursors may play a role in susceptibility to metabolic disease. Further, subcutaneous ASC secrete more adiponectin and have reduced lipolytic rate relative to visceral (Baglioni et al., 2012). Additional metabolic studies show that ASC from both depots use both glycolysis and oxidative phosphorylation for energy production; however, visceral ASC mostly use glycolysis. This suggest that the difference in adipogenic potential is due to the difference in mitochondrial activity (Lefevre et al., 2019).

\subsection{Brown Adipose Tissue (BAT)}

BAT is a multilocular adipose tissue with densely packed mitochondria, enriched in the suprascapular region of the upper neck and mostly found in infants. The main function of BAT is to increase energy expenditure and generate heat by non-shivering thermogenesis. BAT uses stored fat as its substrate upon cold exposure or adrenergic stimulation (Cannon and Nedergaard, 2004). BAT uses uncoupling protein1 (UCP1), activated by long chain fatty acids to uncouple the mitochondrial respiratory chain from ATP production by proton leak in the inner mitochondrial membrane to generate heat and maintain reactive oxygen species (ROS) production. This includes transcriptional activation of the genes encoding UCP1. UCP1 is involved in the oxidation of metabolic substrates and helps maintain glucose and lipid homeostasis (Stanford and Goodyear, 2013). Several transcription factors like PGC1 $\alpha$ and PRDM16 are characteristically expressed in brown adipocytes and play a major role in mitochondrial biogenesis.

\subsection{Brite Adipose Tissue}

Birte adipocytes have multilocular morphology, express UCP1 and resemble brown-like cells within WAT with characteristics, functions, and gene signatures of classical brown 
adipocytes. This phenotype can be induced upon cold exposure or $\beta$ - adrenergic signaling or TZD administration (Rosenwald and Wolfrum, 2014). However, transcriptome analysis showed that brite adipocytes express some unique markers, Tmem26, Tbx1, Cd137 that are not expressed in the classical brown adipocyte marker sets Zic1, Prdm16, Lhx8, Cox8b etc (Waldén et al., 2012; Wu et al., 2012). Given the distinct function of white and brown adipocytes, "white to brite adipocyte" conversion may be due to trans-differentiation without going back to the undifferentiated state. The britening in WAT has been shown to improve overall metabolic profile including reduced adiposity and improved glucose homeostasis (Guerra et al., 1998; Golozoubova, Hohtola, et al., 2001; Golozoubova, Cannon, and Nedergaard, 2006; L. Cao et al., 2011).

\subsection{Roles of Mitochondria in Adipose Tissues and Metabolism}

Mitochondria play an important role in the regulation of energy expenditure, ATP production and disposal of ROS by regulating fatty acid oxidation (FAO) and glucose homeostasis. Normally, adipose tissues maintain the appropriate balance between energy release and storage and maintain normal mitochondrial function. With obesity, excess caloric intake such as HFD increases the mitochondrial substrate load to all tissues involved in nutrient metabolism (adipose, liver, and skeletal muscle) leading to mitochondrial dysfunction. This increases oxidative stress and the production of ROS which will ultimately cause cell damage and inflammation (Y. Lin et al., 2005). ROS will reduce oxygen consumption and block fatty acid oxidation resulting in lipid accumulation. Treatment of 3T3L1 adipocytes with glucose or NEFAs increase ROS, reduces mitochondrial biogenesis and cause insulin resistance (Gao et al., 2010).

Mitochondrial biogenesis and activity play an important role and can have different effects on adipocyte lipid metabolism. Several mitochondrial enzymes play important roles in lipid metabolism. In case of energy demand, WAT releases NEFA by lipolysis which is utilized as a substrate for FAO in liver and skeletal muscle and improves insulin sensitivity. However, in the case of nutrient excess for an extended period, WAT lipogenic enzymes use energy substrate to store triglycerides, leading to NEFA accumulation, mitochondrial dysfunction, and insulin resistance. BAT and muscle cells, shares a common precursor, have high oxidative activity due to higher mitochondrial density with similar expression profile of FAO enzymes and OXPHOS components. Yet each cell type has uniques markers that define the cellular phenotypes, including UCP1, which is unique to BAT (Bournat and C. W. Brown, 2010).

\subsection{Adipocyte Hypertrophy and Hyperplasia}

Obesity is not only an increase in adipose tissue mass, but this is a result of both fat accumulation and fat distribution. Increase in adipose tissue mass can be caused by enlarged 
adipocyte size known as hypertrophy or an increase in adipocyte number during adipogenesis termed as hyperplasia (Choe et al., 2016; Longo et al., 2019). With obesity, WAT may expand in an unhealthy or healthy way. Unhealthy expansion of WAT through hypertrophy can lead to obesity associated metabolic complications such as hypoxia and mechanincal reduction of the blood supply in the tissue, increased ROS production and inflammation with more recruitment of macrophages. This also results in altered adipokine secretion and mitochondrial dysfunction, type 2 diabetes, insulin resistance and non-alcoholic fatty liver disease. Healthy WAT expansion is metabolically beneficial, achieved via recruitment of new adipocytes from precursors by differentiation. Alteration in preadipocyte differentiation is associated with metabolic complications (Lynes and Y. H. Tseng, 2018; Longo et al., 2019)

\subsection{Adipose Tissue Macrophages (ATMs)}

Macrophages and other immune cells including CD4+ and CD8+ T cells, dendritic cells and neutrophils play a crucial role in maintaining adipose tissue function. Hypertrophic adipose tissue is associated with inflammation in which the number and composition of macrophages and other immune cells abnormally changes. From healthy state to obese state transition, the macrophages population increase from $5 \%$ to $50 \%$ within WAT, membrane potential weakens and surrounds dead adipocytes to make crown-like structures in both mice and humans. These macrophages change the inflammatory feature from an antiinflammatory state (M2) in lean individuals to a proinflammatory (M1) state during obesity. These macrophages also contribute to the development of insulin resistance in obesity and activate transcriptional cascade involved in lipolysis (X. Xu et al., 2013; Boutens and Stienstra, 2016).

\subsection{An Overview of Activin Signaling}

The TGF- $\beta$ family is comprised of over 33 ligands including TGF- $\beta$, activins, nodal, bone morphogenic proteins (BMPs) and growth differentiation factors (GDFs) (Poniatowski $Ł$ et al., 2015). These ligands are divided into two major groups: TGF- $\beta$-like ligands including activins and BMP-like ligands. Activins initiate signaling through a combination of type I and II transmembrane serine/threonine kinase receptors. Many signaling pathways use cascades of cytoplasmic molecules as relay transducers. The binding of TGF- $\beta$ family ligands with receptors and activation of downstream SMADs depend on the receptor with which they interact (Zi, Chapnick, and X. Liu, 2012). SMAD2 and SMAD3 are the cytoplasmic downstream effector proteins of activin signaling (Fei et al., 2010; Tsurutani et al., 2011; Zamani and Chester W. Brown, 2011; Yadav, Quijano, et al., 2011; L. Zhang et al., 2015; Namwanje and C. W. Brown, 2016; Xue et al., 2016; Wankhade et al., 2018; Q. Zhu et al., 2018; Aragon et al., 2019; D. Wang et al., 2020). Classically, activins signal through activin receptors and downstream transcriptional transducers SMAD2 and SMAD3. 
This drives a variety of activin functions during embryonic development and in adult tissues including fat cells, where activins regulate proliferation, adipocyte differentiation, cell fate determination, the management of body fat composition and energy homeostasis (Waldrip et al., 1998; Y. Zhu et al., 1998; Ashcroft et al., 1999; Datto et al., 1999; C. W. Brown, L. Li, et al., 2003; Dunn et al., 2004; Hirai et al., 2005; Ju et al., 2006; Itman et al., 2009; Fei et al., 2010; Zaragosi et al., 2010; C. K. Tan, Leuenberger, et al., 2011; Tsurutani et al., 2011; Yadav, Quijano, et al., 2011; Fleming et al., 2013; C. K. Tan, E. H. Tan, et al., 2013; Kashiwagi et al., 2015; L. Liu et al., 2016; Xue et al., 2016; Y. P. Zhu et al., 2017; Aragon et al., 2019). Activin/SMAD signaling is in part regulated by changes in equilibrium between the cytoplasm and the nucleus (Caroline S. Hill, 2016).

Activin A $(\beta A)$ and activin $B(\beta B)$ ligands associate with activin receptors type IIA or type IIB (ActRIIA or ActRIIB) and activate them to phosphorylate and activate type I activin receptors (ActRI) such as activin receptor like kinase (ALK4 and ALK7). Activated ALK4/ALK7 then transduce signal by phosphorylating the C-terminal Ser-Ser-XSer (SSXS) motif of regulatory SMADs (R-SMADs) SMAD2 and/or SMAD3. SMAD proteins undergo a continuous cycle of nucleocytoplasmic shuttling. C-terminal phosphorylation of SMAD2/3 allow interaction with the common mediator SMAD (Co-SMAD), SAMD4 to form heteromeric complexes and then translocate into the nucleus. In the nucleus, the heteromeric complexes bind to the regulatory elements of target genes and regulate transcription by transcription factors, transcriptional co-activators, or co-repressors to impact a verity of biological function during embryonic development and in adult tissues, including cell proliferation, differentiation, and cell fate determination. BMPs binds to BMP receptors and signal transduction occurs via SMAD1/5/8 (J. Massagué, 1998; Joan Massagué, 2000; Chang, C. W. Brown, and Matzuk, 2002; Morikawa, Derynck, and Miyazono, 2016; Derynck and Budi, 2019; Olsen et al., 2020)(Figure 2.2). SMADs activity is also controlled by other posttranslational modifications such as sumoylation, that affect protein stability and transcriptional activity (K. Tsuchida et al., 2004). SMAD6 and SMAD7 (inhibitory SMAD) disrupt the binding of R-SMADs with co-SMAD. TGF- $\beta$-like ligands including activin can also mediate the signal via non-SMAD mediated pathways such as p38MAPK, ERK1/2 and JNK depending on the tissue type (Engel et al., 1999; Hanafusa et al., 1999; Fu et al., 2003; W. Cao et al., 2004; J. Hu et al., 2016).

\subsection{Adipogenesis and Key Transcription Factors Regulating Adi- pogenesis}

Adipogenesis is a multistep temporal process of differentiation of preadipocytes into mature adipocytes that includes early clonal expansion of preadipocytes and committed differentiation(Ailhaud, Grimaldi et al. 1994). For example, differentiation of pluripotent embryonic stem cells (ESCs) into adipocytes starts with cell fate commitment into multipotential mesenchymal progenitors (MSCs) such as C3H10T1/2 cells and MEFs. MSCs undergo cell fate determination and commitment to the preadipocyte, osteoblast, chondroblast or myoblast 
lineage(Gregoire, Smas et al. 1998, Hausman, DiGirolamo et al. 2001, Tang, Otto et al. 2003, Ghaben and Scherer 2019). Under the appropriate adipogenic culture conditions, committed preadipocytes (3T3-L1, 3T3-F422A or primary preadipocytes) undergo mitotic clonal expansion in which growth-arrested preadipocytes undergo a single round of cell division before beginning the process of differentiation into mature adipocytes (Tang, Otto, and Lane, 2004). Pref and Wnt10b expression are high in proliferating preadipocytes and inhibit adipogenesis by inhibiting the expression of Cebp $\beta$ and Cebp $\delta$ by increasing the expression of Sox9 (H. S. Sul et al., 2000; Y. Wang, K.-A. Kim, et al., 2006; Y. Wang and Hei Sook Sul, 2009; Cawthorn et al., 2012). This process is regulated by several, temporal transcriptional events, including the sequential expression of adipogenic CCAAT/enhancer binding protein transcription factors $\mathrm{C} / \mathrm{EBP} \alpha, \mathrm{C} / \mathrm{EBP} \beta, \mathrm{C} / \mathrm{EBP} \delta$ and Ppar $\gamma$ (Farmer, 2006). Differentiation is initiated by adding a hormonal cocktail, which induces Cebp $\beta$ and Cebp $\delta$. Subsequently, Cebp $\alpha$ and Ppar $\gamma$ are expressed, leading to terminal differentiation of mature adipocytes, characterized by the expression of Fabp4, Lep and other markers (E. D. Rosen, Hsu, et al., 2002; E. D. Rosen and Spiegelman, 2006; Zamani and Chester W. Brown, 2011). In the process of transition from mitotic clonal expansion to terminal differentiation, FOXO1 is activated, which in turn activates p21 (a cell cycle inhibitor), leading to the 2nd growth arrest and permitting terminal differentiation (Fajas, 2003; Nakae et al., 2003; Seoane et al., 2004; Munekata and Sakamoto, 2009) (Figure 2.1). During adipogenesis, mitochondrial number and mitochondrial activity greatly increases.

The mature adipocyte is a key regulator of energy and glucose homeostasis. The primary function of the adipocyte is to accumulate triglycerides during excess caloric intake and to release non-esterified free fatty acids (NEFA) during periods of energy demand. Adipocytokines secreted by the adipocyte and other cell types within adipose tissue play a crucial role in maintaining energy homeostasis. Mature adipocytes regulate numerous key factors including regulating the balance between the fatty acid synthesis (lipogenesis) and breakdown of triglycerides (lipolysis) and synthesis of non-esterified fatty acids (NEFA). Hormone sensitive lipase (HSL) and adipocyte triglyceride lipase (ATGL) are the main lipases for the regulation of lipolysis (Holm, 2003)). $\beta$-adrenergic stimulation of lipolysis requires HSL activation (Haemmerle et al., 2002; Fortier et al., 2004; Duncan et al., 2007; Frühbeck et al., 2014). Also, cAMP-dependent PKA-mediated phosphorylation of PERILIPIN and HSL leads to the conformational change that allows the contact of triglycerides with HSL, which releases NEFA and glycerol for transport to the cell surface by fatty acid binding proteins (Coe, Simpson, and Bernlohr, 1999). NEFAs that are taken up by adipocytes during times of energy excess are esterified and form triglycerides (Lehner and Kuksis, 1996; Miyoshi et al., 2006). Insulin inhibits lipolysis through phosphorylation (Evan D. Rosen and Ormond A. MacDougald, 2006). 


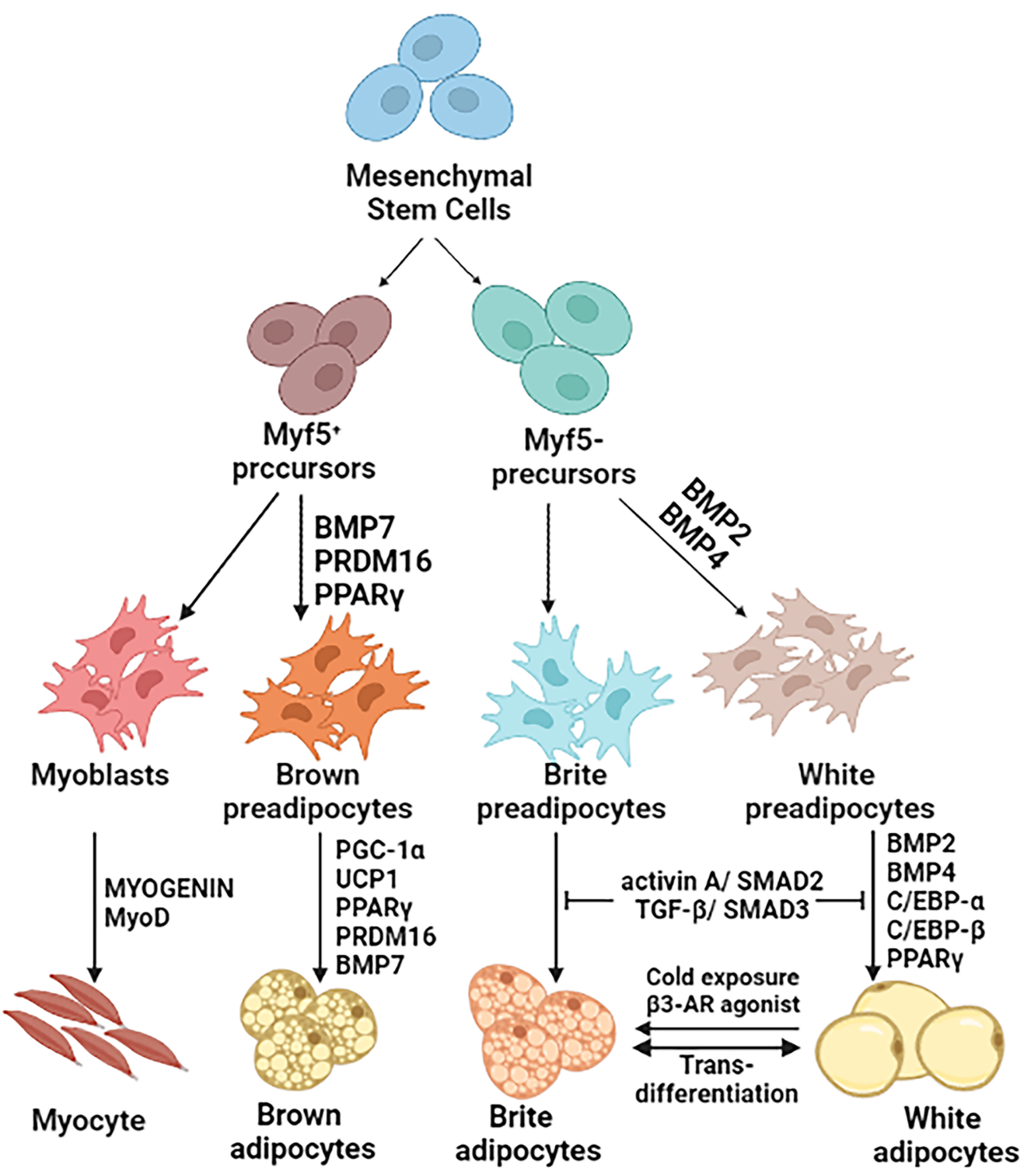

Figure 2.1: Adipogensis. Mesenchymal stem cells and its delineation into Myf5- or Myf5+ precursors and further differentiation into myocytes or brown adipocyte or white or brite adipocytes. Image generated with biorender. 


\subsection{Activin Signaling and Transcription Factors (SMAD2 and SMAD3) Regulate Adipogenesis}

Several TGF- $\beta$ family members including activins A and B play an important role in preadipocyte determination, adipocyte differentiation and function, and consequently, they have direct effects on adipose tissue function (C. W. Brown, L. Li, et al., 2003; Hirai et al., 2005; L. Li et al., 2009; Namwanje and C. W. Brown, 2016; Xue et al., 2016; Wankhade et al., 2018). Some of the ligands in this family such as BMP2, BMP4, BMP7 stimulate adipocyte differentiation are also involved in the commitment of MSC precursors (C3H10T1/2) or 3T3-L1 preadipocytes to the adipocyte lineage (Tang, Otto, and Lane, 2004; Bowers et al., 2006). BMP7 supports brown adipogenesis by inducing the expression of genes involved in mitochondrial biogenesis, while TGF- $\beta$ and activins have inhibitory roles on adipogenesis (Y.-H. Tseng et al., 2008; Zamani and Chester W. Brown, 2011). Activins A and B exert pleiotropic actions on adipose tissue function (C. W. Brown, L. Li, et al., 2003; Hirai et al., 2005; L. Li et al., 2009; Xue et al., 2016; Wankhade et al., 2018; M. J. Lee, 2018). Activin A is highly expressed during the early stages of adipogenesis while activin B is highly expressed in mature human adipocytes. Taken together, activin A and B play prominent roles in adipose tissue biology and energy metabolism (Sjöholm et al., 2006; Carlsson et al., 2009; Dani, 2013). Activin signaling during adipocyte differentiation leads to the activation of SMAD2/SMAD3 to inhibit the expression of adipogenic genes, Cebp $\alpha, C e b p \beta, C e b p \delta$ and Ppar (Darlington, Ross, and O. A. MacDougald, 1998). Activin A promotes preadipocyte proliferation and inhibits SMAD2-mediated differentiation during the early stages of human adipocyte progenitor differentiation and in mouse 3T3-L1 cells by reducing Ppar $\gamma$ and Cebp $\alpha$ expression (Hirai et al., 2005; Horie et al., 2008; Zaragosi et al., 2010). Hence, downregulation of activin $\mathrm{A}$ is required for the commitment of precursors to the adipocyte lineage (Dani, 2013). Also, activin A/SMAD2 signaling inhibits preadipocyte differentiation and promotes self-renewal of adipose progenitors by inducing expression of Nanog (Q. Zhu et al., 2018). Additionally, activin B inhibits lipolysis in 3T3-L1 cells (Magnusson et al., 2010). Activin can also compete for type II receptors used in BMP pathways that phosphorylate ALK2, thereby antagonizing BMP-induced, SMAD1/5-mediated signaling or in some contexts activating the same pathway. Whether activin-associated SMAD1/5-mediated effects occur in adipose remains unknown (Olsen et al., 2020), image partially adapted from Olsen et al., 2020, biomolecules and generated with biorender (Figure 2.2).

\subsection{Structure and Function of SMAD2 and SMAD3}

In mammals, there are 8 SMAD proteins designated SMAD1-8. These proteins are divided into three groups: the receptor-mediated SMADs (R-SMADs), common mediator SMAD (Co-SMAD), and the inhibitory SMADs (I-SMAD). The R-SMADs include SMAD1, SMAD2, SMAD3, SMAD5 and SMAD8 and are divided into two main branches such that SMAD2 and SMAD3 are transducers for TGF- $\beta$-like ligands (TGF- $\beta$, activins and nodal) and SMAD1, 


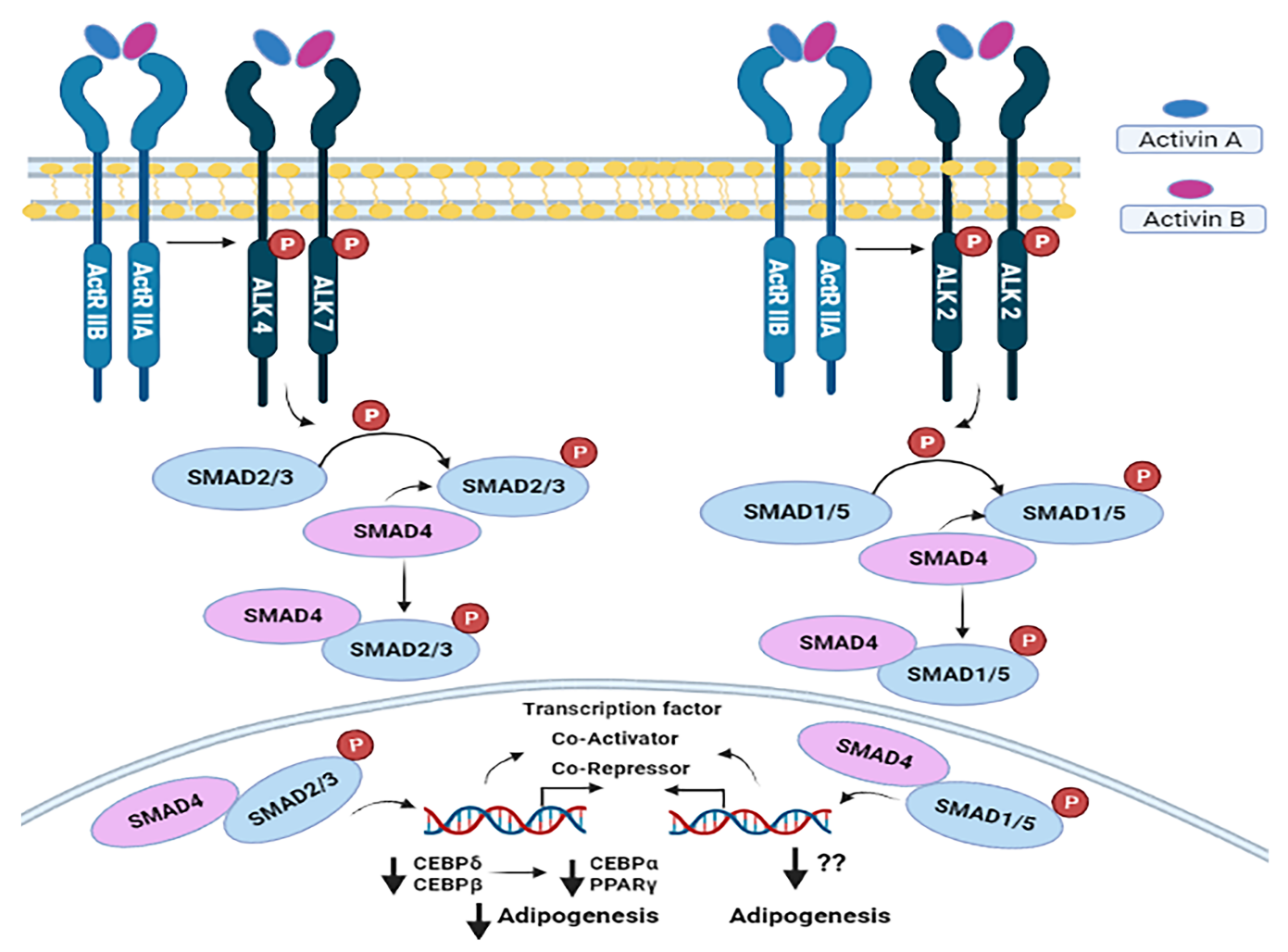

Figure 2.2: SMAD-Mediated Activin Signaling Pathway. Activin A and B bind to the type II receptors, ActRIIA and ActRIIB which phosphorylates ActRI (ALK4/7). Active ALK4/7 further lead to phosphorylation of SMAD2 and SMAD3. pSMAD2 and pSMAD3 makes a complex with SMAD4 and translocate into the nucleus and regulate transcription of adipogenic genes. Activin inhibit adipogenesis via SMAD2/3 pathway by suppressing the expression of Pparr and Cebp $\alpha$, the master regulator of adipogenesis. Activins can also interact with BMP-receptor type I, ALK2 which can phosphorylate SMAD1/5. Phosphorylated SMAD1/5 interacts with SMAD4 and regulates the transcription of target genes which may involve in adipogenesis. Image partially adapted with open access permission from biomolecules, Olsen et al., 2020 (Olsen et al., 2020) and generated with biorender. 
5 and 8 are substrates for the BMP like ligands. The Co-SMAD, SMAD4 a consistent component of R-SAMD-Co-SMAD complexes and associates with all R-SMADs in both TGF- $\beta$ and BMP branches. Inhibitory SMADs, SMAD6 and SMAD7, block the binding of R-SMADs with Co-SMAD or target ligand receptors complexes for degradation in both TGF- $\beta$ and BMP branches (Zamani and Chester W. Brown, 2011).

SMAD2 and SMAD3 are nearly identical proteins of $\approx 500$ amino acids, sharing $\approx 92 \%$ amino acid similarity. SMAD proteins consists of two conserved regions known as the $\mathrm{N}$-terminal Mad homology domain (MH1) and C-terminal Mad homology domain (MH2) coupled by linker region. SMAD2 and SMAD3 proteins have $66 \%$ amino acid similarity at the MH1 domain except SMAD2 has a unique highly conserved 30 amino acid E3 insert in the MH1 domain which is absent in SMAD3. Therefore, SMAD2 has two isoforms, encoded by alternatively spliced transcripts, one lacking the E3 insert (SMAD2 $\beta$ isoform). In contrast, SMAD2 and SMAD3 exhibit $96 \%$ amino acid similarity in the MH2 domains. During signal transduction, SMAD2 and SMAD3 form homodimers or heterodimers and associate with SMAD4 (Aragon et al., 2019). The R-SMADs and SMAD4 have N-terminal nuclear localization signals (NLS) in the MH1 domain while SMAD4 has an additional nuclear export signal (NES) in the linker region (Z. Xiao et al., 2003; K. A. Brown, Pietenpol, and Moses, 2007) (Figure 2.3).

$\mathrm{N}$ - terminal MH1 domains of SMAD2 and SMAD3 are involved in DNA binding while the $\mathrm{C}$-terminal $\mathrm{MH} 2$ domains interact with nuclear proteins including transcriptional co-activators (P300/CBP), co-repressors (p107, c-SKI) and other transcription factors (FOXO $1,3,4)$ which regulate the transcription of TGF- $\beta$ family downstream target genes $(X$. Shen et al., 1998; W. Xu et al., 2000; Seoane et al., 2004; Feng and Derynck, 2005). ISMADs lack MH1 domain (Y. Shi and J. Massagué, 2003). Though it has been stated that SMAD2 and SMAD3 show 92\% sequence similarity, they have acquired different functions during development. SMAD3 alone or in a complex with SMAD4 and other interacting partners can bind to the DNA directly and regulate the expression of key genes required for development, while SMAD2 homodimer fails to bind directly to DNA in the absence of SMAD4 because of the additional 30aa E3 insert. Hence, SMAD2/SMAD4 heteromer requires interaction with other transcription factors to form DNA-binding complexes (Joan Massagué, 2000; Moustakas, Souchelnytskyi, and Heldin, 2001; Dijke and C. S. Hill, 2004). Consistent with this idea, FOXH1, POU5F1/OCT4 and FOXO1, 3, and 4 proteins have all been shown to interact with SMAD2 and help to bind to DNA (Seoane et al., 2004; Mullen et al., 2011; Aragon et al., 2019). This indicates that SMAD2 and SMAD3 have evolved unique and non-overlapping functions depending on the interacting partners with whom SMAD2 and SMAD3 engage. Therefore, SMAD2 and SMAD3 may function similarly, with functional overlap in some tissues and cellular contexts but may also have unique and non-overlapping function in other tissues depending on the type of SMAD complex (homotrimeric/heterotrimeric). The specific composition of these SMAD complexes has not been determined for several cell types. Alternative composition of SMAD complexes was further supported by Aragon et al. 2019 when they showed that SMAD2 can also bind 


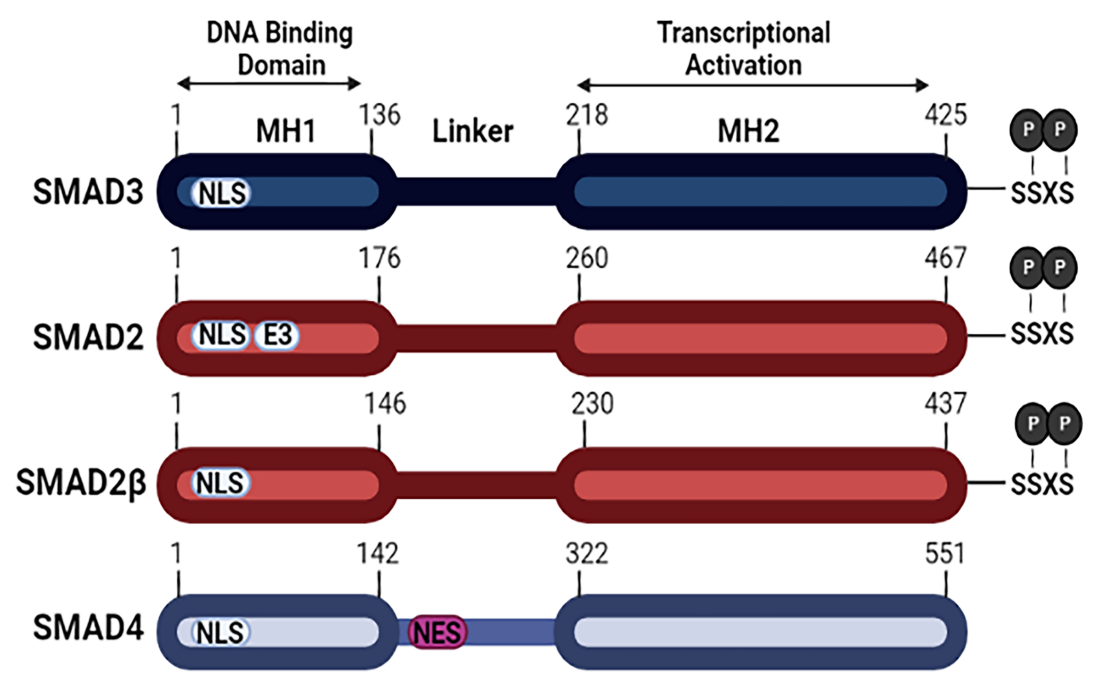

Figure 2.3: Structures of SMAD3, SMAD2, SMAD2 $\beta$ and SMAD4.

SMAD3 and SMAD2 are highly homologus protein having N-terminal Mad homology domain (MH1) and C-terminal Mad homology domain (MH2) coupled by linker region. MH1 domain of SMAD2, SMAD3 and SMAD4 contains nuclear localization signals (NLS) in MH1 domain while common-smad, SMAD4 contains NLS and nuclear export signal (NES)in the linker region. MH1 domain of SMAD2 and SMAD3 differs from each other by 30AA E3 insert in SMAD2 which is absent in SMAD2 $\beta$. MH2 domain has SSXS phosphorylation site which are the direct targets of activin/TGF- $\beta$ signaling. Image generated with biorender. 
to the DNA directly. To do so SMAD2 exists in alternative, tri-dimensional conformations, one with a correctly folded E3 insert that can directly bind to DNA (Aragon et al., 2019). SMAD3/SMAD4 complexes bind multiple 5'-AGAC' sequences known as SMAD binding elements (SBEs) at the promoter of target genes using the MH1 domain (J. Kim et al., 1997; Yigong Shi et al., 1998). Collectively, depending on the affinity of interacting transcription factors with SMAD2 or SMAD3 and whether activin or TGF- $\beta$ signaling occurs, SMAD2 and SMAD3 function differently. This is illustrated by EVI, which functions as a repressor by interacting with SMAD2 during activin signaling and also as a repressor by interacting with SMAD3 during TGF- $\beta$ signaling (Kurokawa et al., 1998; Allison et al., 2008).

\subsection{SMAD2 and SMAD3 in Development and Disease}

Although SMAD2 and SMAD3 are closely related proteins, they have either overlapping or distinct roles depending on the tissue or cell type where these proteins are expressed. In most cell types, SMAD2 and SMAD3 are co-expressed and SMAD2 and SMAD3 global knockout mice have different phenotypes. Both proteins differ from each other in several ways including cellular localization, as activated SMAD2 forms a high affinity complex with SMAD4 that translocates to the nucleus for transcriptional activities, while SMAD3 remains in the nucleus even in the steady state, has weak affinity for SMAD4 and has distinct roles in embryonic development (L. Liu et al., 2016). It has been reported that SMAD2 and SMAD3 regulate many biological processes during embryogenesis and adipose tissue homeostasis, including cell growth, differentiation, and cell fate determination. SMAD2 and SMAD3 have distinct functions such as the prominent role of SMAD2 during embryonic development (Kriseman et al., 2019). Smad2 knockout mice die in utero (7.5) due to defects in mesoderm formation and defects in gastrulation (Nomura and E. Li, 1998; Waldrip et al., 1998; Weinstein et al., 1998; Ju et al., 2006). In contrast to Smad2, Smad3 global knockout mice are viable and fertile however these mice are smaller than their control littermates and undergo growth retardation postnatally with immune defects, forelimb malformation and colorectal cancer. $70 \%$ of mice die between 1 to 3 months and rest die by 8 months of age (Q. Zhu et al., 2018; Ashcroft et al., 1999; Datto et al., 1999; Yang et al., 1999; C. K. Tan, Leuenberger, et al., 2011). Conditional deletion of individual Smad2 or Smad3 in the ovary, using Amhr2Cre caused a moderate change in female fertility, while combined deletion of Smad2 and Smad3 using Amhr2Cre led to significant reduction in female fertility. Using Progesterone receptor $\mathrm{Cre}$ ( $\mathrm{Pgr} \mathrm{Cre}$ ), uterine selective Smad2/3cDKO mice displayed infertility due to endometrial hyperproliferation starting at puberty, which ultimately resulted in uterine cancer with $100 \%$ mortality by 8 months (Q. Li, Pangas, et al., 2008; Kriseman et al., 2019). Furthermore, it has been shown that in liver, deletion of Smad2 or Smad2/3 using Albumin-Cre does not impair liver function, indicating that SMAD2 and SMAD3 are not required for normal liver function. However, hepatocytes isolated from Albumin-Cre, Smad2cKO proliferate faster than wildtype. This indicates SMAD2 functions as a negative regulator of hepatocyte proliferation in liver in vivo which is consistent with findings in a 
liver injury model. Like Smad2, Smad3cKO showed a similar trend in vitro but not in vivo (Ju et al., 2006).

With excess calories, in addition to white adipose tissues, ectopic fat deposition can take place in other metabolically active organs such as pancreas, liver, and muscle. To examine the role of TGF- $\beta$ /SMAD3 in different organs, Sushil Rane's group studied all metabolically relevant organs. Yadav et al., (Yadav, Quijano, et al., 2011), Tan et al., (C. K. Tan, Leuenberger, et al., 2011) and Tsurutani Y. et al (Tsurutani et al., 2011) have demonstrated that the global knockout of Smad3 in mice protects them from diet-induced obesity and improves their overall metabolic profile including glucose tolerance and insulin sensitivity with reduced WAT mass. Also, Smad3 global knockout mice had altered phenotypic characteristics of WAT, including multilocular, UCP1 positive adipocytes and other molecular features of brown adipose tissue, with increased mitochondrial biogenesis (Yadav, Quijano, et al., 2011). SMAD3 negatively regulated serum IRISIN secretion by reducing the expression of $F N D C 5$ and $P G C-1 \alpha$ at the transcriptional as well as protein levels in skeletal muscle (Tiano, Springer, and Rane, 2015). Additionally, deletion of Smad2 in pancreatic $\beta$-cells resulted in islet cell hyperplasia with impaired INSULIN secretion (Nomura, H. L. Zhu, et al., 2014), where as Smad3 deletion enhanced insulin secretion by regulating genes involved in pancreatic $\beta$-cell function (H. M. Lin et al., 2009). Liver-selective deletion of Tgf- $\beta$ RI or Smad3 suppressed liver gluconeogenesis under normal physiological conditions and type 2 diabetes via PP2A-AMPK-FOXO1. Additionally, inhibition of TGF- $\beta$ /SMAD3 signaling by shSmad3, or TGF- $\beta$ (Hildebrandt et al., 2009) inhibitors (SB431542) in vitro resulted in suppressed glucose levels in hepatic cells (Yadav, Devalaraja, et al., 2017).

\subsection{Role of Microbiota on Obesity}

Gut microbiomes play an important role in metabolic disease, including obesity, type 2 diabetes and inflammation (Awan, Meurling, and O'Shea, 2021). The microbiome regulates host energy homeostasis, including adipose tissue induced by HFD. High fat diet promotes metabolic disorders by altering the gut microbial community including the changes in the proportion of Bacteroidetes, Firmicutes and probacteria. Damage to the gut barrier and overall changes in gut microbiome contribute to obesity and development of other metabolic diseases such as type2 diabetes in mice (C. Zhang et al., 2012). Bacteroidetes and Firmicutes comprise the major gut microbiome and their proportion is a major determinant in obesity (Turnbaugh, Ley, et al., 2006). Studies have shown dose-dependent effects of HFD on gut microbial changes (Carmody et al., 2015). In a rat model, it has been shown that obese rats have different gut microbiota than obesity resistant rats on the same HFD. Transplantation of gut microbiome from obese mice into germ free mice resulted in an obese phenotype (Duca et al., 2014). Additionally, changes occur in the composition of the microbial community under prolonged cold exposure resulting in an increase in the Firmucutes/ Bacteroidetes ratio by 3.5 fold compared to mice at room temperature (Chevalier et al., 2015). The mechanisms involved in the observed phenotype including 
inflammation, intestinal permeability and upregulated lipogenesis are dependent upon the HFD composition. Additionally, gut microbiota are crucial in modulating lipogenic genes (E. Y. Huang et al., 2013). Targeting gut microbiome may reduce the risk of obesity and associated metabolic disease (Murphy, Velazquez, and Herbert, 2015).

The gut microbiome communicates with adipose tissue, regulates energy homeostasis, and contributes to the development of obesity. Juge et al. suggested that the composition and activities of the gut microbiome contributes to obesity. The gut microbiome regulates absorption of nutrients in the intestine and regulates adipose tissue energy homeostasis and thermogenesis through short-chain fatty acids (SCFAs)(Thursby and Juge, 2017; H. Xiao and Kang, 2020). Bacterial metabolites such as SCFAs and bile acids influence the function of adipose tissue and contribute to the regulation of energy homeostasis of adipose tissue. Gut microbiome produced butyrate, a SCFA, impacts the thermogenic activity of brown adipose tissue, as administering sodium butyrate to microbiota-depleted mice partially rescued impaired thermogenesis and promoted fatty acid oxidation. Studies also demonstrated that butyrate from the gut is delivered to the brain more so than other tissues and that gut-derived butyrate activates WAT britening through a gut-brain circuit but may not exert direct effects within adipose (B. Li et al., 2019).

Genetic manipulation or cold exposure alters the diversity of gut microbiota in mice, altering microbial metabolites (butyrate and other SCFAs) and conferring thermogenic properties. Transfer of the cold microbiota in germ-free mice resulted in thermogenic ability with a corresponding increase in energy expenditure. Additionally, transplantation of cold microbiota induced browning, promoted thermogenesis in WAT and improved insulin sensitivity (Chevalier et al., 2015; Ziętak et al., 2016). Additionally, butyrate increased energy expenditure and mitochondrial activity by increasing $\operatorname{Pgc} \alpha$ in brown adipose tissue. Further, butyrate treatment increased energy expenditure genes including mitochondrial genes and browning genes such as Ucp1, Pgc1 $\alpha$, Cpt1b, Cox7a in WAT and BAT (D. Wang et al., 2020).

To understand the mechanism(s) underlying the role of the microbiome in obesity, the microbiome of human twins (one obese and one lean) were transplanted into germ free mice. These mice were fed low fat, high carbohydrate diet. The recipient mice of obese microbiota gained more fat, with more Firmicutes expressing genes involved in nutrient transporters. In contrast, mice receiving lean microbiota, with more Bacteroidetes expressing genes involved carbohydrate metabolism. These difference in body fat were correlated with a different capability for fermenting nutrients such as lean mice had more fermentation of SCFAs while obese mice most of the metabolites from branched chain amino acid (Turnbaugh, Ley, et al., 2006; Turnbaugh, V. K. Ridaura, et al., 2009; Turnbaugh, Hamady, et al., 2009; Vanessa K. Ridaura et al., 2013; O’Keefe, 2016; H. Xiao and Kang, 2020).

The relationships among the gut microbiome, obesity, and TGF- $\beta$ /SMAD3 signaling are emerging. Smad3 global knockout mice exhibit growth retardation and develop colorectal cancer. Daniel et. al demonstrated the link between the development of colon cancer and 
gut microbiome, specifically Helicobacter in Smad3 global knockout mice. Smad3 deficient mice with colon cancer displayed functional changes in the gut microbiome. Using an omics approach, this group has shown the functional differences/shifts in the microbiome, specifically the bacterial communities involved in distinct pathways including colon cancer, LPS production, SCFA metabolization and oxidative phosphorylation in Smad3 global knockout mice with or without inoculation of $\mathrm{H}$. hepaticus. Upregulation of Bacteroidaceae by 1.89 fold was observed alone and 2.26 fold upregulation with $\mathrm{H}$. hepaticus in Smad3 global knockout mice compared to wild type mice. A similar trend was observed at the phylum level of Bacteroidetes. The Smad3 deletion associated inflammation is not the only cause of tumor formation, as Helicobacter alone is strongly associated with colon inflammation and Helicobacter triggers inflammation in Smad3 global knockout mice. In addition to Helicobacter, several other bacterial species are involved in the development of colon cancer in Smad3 global knockout mice (Daniel et al., 2017).

The contribution of intestinal microbiota in inflammatory bowel disease (IBD) is known. The TGF- $\beta /$ SMAD3 pathway is crucial in regulating microbiome associated inflammatory responses and tissue repair and its contribution to IBD. Smad3 global knockout mice are susceptible to the development of IBD. Microbiome transfer from IBD Smad3 global knockout mice to germ free mice led to the induction of IBD, with weight loss and more severe inflammation in the colon in recipient, germ free mice, suggesting a causal role of microbiome in IBD (Paik et al., 2020). 


\section{Chapter 3}

\section{Material and Methods}

\subsection{In Vivo Assay}

\subsubsection{Generation of Mice}

All animal procedures were carried out in accordance with the UTHSC guidelines on the humane treatment of experimental animals and with the explicit approval of the Institutional Animal Care and Use Committee (IACUC). Smad2 flox/flox mice used in this study were purchased from Jackson Laboratories (Ju et al., 2006) (JAX, Bar Harbor, ME, Smad2tm1.1Epb, Stock No 022074). Adiponectin-Cre (B6;FVB-Tg(Adipoq-cre)1Evdr/J), mice were also purchased from Jackson Laboratories (Eguchi et al., 2011) (JAX, Bar Harbor, ME, Stock No 028020).

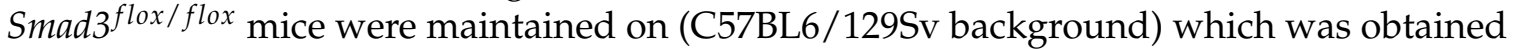
from the Matzuk Laboratory (Matzuk, 1995; Sarjeant and Stephens, 2012). Inhbb-/- (activin B) null mice and Inhba-/- (activin A) null mice were detailed by Vassalli et al. and Matzuk et al., respectively (Schrewe et al., 1994; Vassalli et al., 1994; Matzuk, 1995). Smad2 conditional knockout (Smad2cKO), Smad3 conditional knockout (Smad3cKO) and Smad2/3 conditional double knockout (Smad2/3cKO) mice were generated by crossing Smad2 flox/flox (loxP sites flanking exon2), Smad3 ${ }^{\text {flox } / \text { flox }}$ (loxP sites flanking exon 2-3) and Smad2/3flox/flox mice with Adiponectin-Cre mice. Smad2 flox/flox,$S m a d 3^{\text {flox } / \text { flox }}$ and Smad2/3 $3^{\text {flox } / \text { flox }}$ mice were used as a control to compare for $S m a d 2 c K O, S m a d 3 c K O$ and $S m a d 2 / 3 c K O$ mice respectively. Mice used for the diet study were 5-week-old males and females. Male and female mice were housed and fed standard chow diet (Envigo Lab Diet 7912) until age 5 weeks under an approved IACUC protocol by UTHSC animal care unit. The Low fat-diet (LFD, stock D12450J) and high fat-diet (HFD, stock D12492) feeding started at week 5, purchased from Research Diets Inc., New Brunswick, NJ. Body weight measurements started at 5 weeks and were monitored weekly for 23 weeks. Food intake was measured manually at 16 weeks. Smad2 and Smad3 and Smad2/3 conditional knockout mice were generated using Adiponectin-Cre.

\subsubsection{Glucose and Insulin Tolerance Tests}

Mice were fasted for 6 hours, and baseline blood glucose levels were obtained. $1.5 \mathrm{mg}$ of glucose/gram of body weight for GTT and $0.75 \mathrm{mlU}$ of insulin (humulin-U100)/gram of 
body weight for ITT was injected intraperitoneally, nicked tail vein blood was collected, and blood glucose was measured at the indicated time points using a glucometer (Contour Next glucometer and text strips).

\subsubsection{Body Composition Measurement}

Body composition (fat mass, lean mass and water content in grams) was measured using an EchoMRI 1100 system (EchoMRI, Houston, TX) and body composition was normalized to total body weight. Tissues were harvested for molecular analysis and histology.

\subsubsection{Indirect Calorimetry (Comprehensive Laboratory Animal Monitoring Sys- tem, CLAMS)}

Smad2/3cKO male and female mice on HFD were weighed and housed individually in a metabolic cage with water bottle and ad libitum food, compatible for CLAMS (Columbus Instruments) at $22^{\circ} \mathrm{C}$. Instrument calibration was done against a standard gas mixture of oxygen, carbon dioxide, and nitrogen. Mice were in acclimation period to the new environment for first 24-hours and data collection began after 24-hours of acclimation period and continued for 7 days following 12:12h light/dark cycle. Oxygen consumption $\left(\mathrm{VO}_{2}\right)$, carbon dioxide production $\left(\mathrm{VCO}_{2}\right)$, total energy expenditure (TEE), respiratory exchange ratio (RER) and physical activity were measured via open-circuit indirect calorimetry by Oxymax software (Columbus Instruments). In fasting conditions, initial 6-hours data was excluded from the time of fasting. Total energy expenditure (TEE) was calculated using Lusk equation(Lusk), TEE (heat) $=(3.815+1.232 \times \mathrm{RER}) \times \mathrm{VO}_{2}$. Total physical activity was calculated as the combined number of infrared beam breaks in both $X$ and $Y$ axis. Ambulatory activity was calculated by combining the number of consecutive $X$ and $Y$ axis beam breaks.

\subsubsection{DNA Isolation and Genotyping}

Genomic DNA was isolated from tail biopsies using standard alkaline lysis procedure. Go Taq Green master mix (Promega. Cat M7123) with addition of $2 \mathrm{mM} \mathrm{MgCl} 2$ based genotyping was performed using a Veriti 96-well Touch Thermal cycler (Applied Biosystems Life technologies). The Smad2 floxed allele was detected using the primers following the protocol from Jackson Laboratories: Smad2 forward primer: AGC TTG AGA AAG CCA TCA CC and reverse primer GAC CAA GGC GAA AGG AAA CT. The product size was $350 \mathrm{bp}$ for WT and 400bp for the floxed allele and Smad3 forward primer: CTCCAGATCGTGGGCATACAGC and reverse primer GGTCACAGGGTCCTCTGTGCC. The product size was $200 \mathrm{bp}$. The activin $A$ null allele was detected using the primers HPRT-A forward - 5' - GACCTCTCGAAGTGTTGGATAC-3' and HPRT-B reverse - $5^{\prime}$ CTTGCGCTCATCTTAGGCTT-3', PCR product size $170 \mathrm{bp}$. The following amplification scheme was used with $5 \%$ DMSO in the above PCR mix: $98^{\circ} \mathrm{C}$ for 5 min for 1 cycle; $98^{\circ} \mathrm{C}$ for $30 \mathrm{sec}, 50^{\circ} \mathrm{C}$ for $30 \mathrm{sec}, 72^{\circ} \mathrm{C}$ for $1 \mathrm{~min}$ for 35 cycles; $72^{\circ} \mathrm{C}$ for $10 \mathrm{~min}$ 
and then $4^{\circ} \mathrm{C}$ to hold. The corresponding WT allele was detected using the primers BA EXON3 forward - 5'-ACAGAACCAGGACCAAAGTCACCA-3'; BA EXON3 reverse, 5'-TCCAGTCATTCCAGCCAATGTCCT-3', the product size $560 \mathrm{bp}$. WT PCR amplification reaction was performed without DMSO and the annealing temperature was set at $58^{\circ} \mathrm{C}$. Presence of either HPRT or WT band alone indicated the null allele for activin A and WT respectively; however, presence of both bands indicated Het samples for activin A. The activin $B$ null allele was detected using the multiplex PCR assay primers (P1, P2 and P3): P1 BB 5' PRO 2 5'-ATGGTCACGGCCCTGCGCAA-3', P2: BB 3' Exon1 5'CAAAGCTATGATCTCGGAGA-3' ${ }^{\prime}$ and P3: Neo forward $5^{\prime}$ - CCTTCTTGACGAGTTCTTCT$3^{\prime}$. These 3 primers generated PCR products of $150 \mathrm{bp}$ or $500 \mathrm{bp}$, corresponding to the WT and activin $\mathrm{B}$ null alleles, respectively. PCR amplification reactions were carried in the following conditions: 35 cycles consisting of $50 \mathrm{sec}$ of annealing at $55^{\circ} \mathrm{C}$ (no DMSO) and the rest was similar to above PCR conditions.

\subsubsection{Tissue Collection}

Visceral and subcutaneous adipose tissues were dissected, weighed, and processed for RNA, protein and histology.

\subsubsection{Histology}

Visceral and subcutaneous WATs were fixed in $4 \%$ paraformaldehyde at $4{ }^{\circ} \mathrm{C}$ overnight, embedded in paraffin and sectioned at 5 microns. After deparaffinizing, rehydrated sections were stained with hematoxylin and eosin (Cat. No. H-3502, Vector Laboratories) following the vendor's protocol. Images were acquired using EVOS FL Auto Imaging System (Thermo Fisher, Foster City, CA).

\subsubsection{RNA Isolation and Gene Expression Analysis}

Total RNA was isolated from visceral and subcutaneous adipose tissues or undifferentiated and differentiated cells using the trizol method (Invitrogen). cDNA was prepared according to the manufacturer's protocol using the Invitrogen Superscript IV First-Strand Synthesis System kit (Invitrogen 18-091-050). Quantitation of gene expression was performed by real-time RT-PCR (Quant Studio 6 Flex Real-Time PCR System, Applied Biosystems) using SYBR Green master mix (Applied Biosystems), normalized to Cyclophilin B. The quantitative analysis was performed using the standard delta-delta ct method and results are shown as relative fold change in gene expression compared to wild type. Analysis of quantitative PCR data was accomplished using Relative Expression Software Tool (REST) 2008 (Corbett Research, Technical University, Munich, Germany) and GraphPad. The REST analysis addresses issues regarding the measurement of uncertainty in expression ratios inherent in quantitative PCR analysis by using randomization and boot-strapping techniques, including 50,000 hypothesis tests to achieve a high level of consistency. 


\subsubsection{Western Blot Analysis}

Protein lysates were prepared from tissues, undifferentiated and differentiated MEFs and preadipocytes after homogenization in tissue or mammalian protein extraction reagent (TPER for tissue (catalog 78510) and mPER, for cells (catalog 78501)), supplemented with phosphatase and protease inhibitors. Cell lysates were cleared by spinning down at $17000 \mathrm{~g}$ for 30 minutes at $4^{\circ} \mathrm{C}$, then placed on ice to separate lipids from tissue and re-centrifuging for 10 minutes. Protein quantity was measured using the Pierce BCA-protein assay kit (Thermo Fisher Scientific Inc.). Equal amounts of protein (30 $\mu \mathrm{g} /$ well) were loaded on 4-12\% SDS polyacrylamide gels and transferred to PVDF membranes. Blots were blocked in 5\% nonfat dried milk in tris-buffered saline with 0.1\% Tween-20 (TBST) for $1 \mathrm{hr}$. Membranes were washed briefly with TBST, then incubated overnight with the following primary antibodies at $4^{\circ} \mathrm{C}$ : SMAD2 (Cell Signaling, CS3122), SMAD3 (Cell Signaling, CS9523), pSMAD2 (Cell Signaling, CS3108, CS3104), pSMAD3 (Abcam, ab52903, Cell Signaling, CS9520), SMAD2/3 (Cell Signaling, CS8685) and GAPDH (Santa Cruz, SC32233) in 2\% BSA diluted in 1X TBST. The following day, membranes were washed with TBST three times, 10 minutes each, then incubated with HRP- conjugated secondary antibody (Cell Signaling, 7074, Santa Cruz, SC516102) for $1 \mathrm{hr}$. Membranes were then washed thrice with TBST. GAPDH protein was used as loading control for each assay. Finally, protein bands were detected using a chemiluminescence imager (Amersham imager 600).

\subsection{In vitro Assay}

\subsubsection{Mouse Embryo Fibroblasts (MEFs) Isolation and Cell Culture}

From timed mating, MEFs were isolated from E13.5 embryos of WT and activin dKO mice. First, embryos were isolated and transferred to a new dish (one dish per embryo to avoid genotyping cross-contamination) containing 1X PBS (pH7.4). Using sterile forceps, the head, tail, limbs and abdominal contents were removed. Tail/limbs were saved for genotyping. The remaining portion of the embryos were transferred into a sterile Eppendorf tube containing $0.25 \%$ trypsin-EDTA. The tissues were crushed thoroughly with forceps. Tubes were kept on ice during collection and kept at $4^{\circ} \mathrm{C}$ overnight. The tail/limb samples were processed for genotyping of wild type and activin $\mathrm{dKO}$ mice. MEFs were processed as follows: All tissues that were kept at $4^{\circ} \mathrm{C}$ were placed at $37^{\circ} \mathrm{C}$ for 15 minutes. Then tube exteriors were sprayed with $70 \%$ ethanol and using a $1 \mathrm{ml}$ pipette filtered tip, the tissues were gently moved up and down 3 times and transferred to $15 \mathrm{ml}$ tubes containing $5 \mathrm{ml}$ basic medium (1X DMEM supplemented with 10\% FBS (Hyclone), sodium pyruvate (1 $\mathrm{mmol} / \mathrm{L}), 1 \%$ Penicillin/Streptomycin (100X), and L-glutamine (2 mmol/L). A vigorous mixing with a $5 \mathrm{ml}$ pipette was then performed to disintegrate the tissues, which were then filtered at $100 \mu \mathrm{m}$ into a sterile $50 \mathrm{ml}$ conical tubes. The filtered suspensions containing MEF cells were transferred to $10 \mathrm{~cm}$ culture dishes, medium adjusted to a total of $10 \mathrm{ml}$, and were cultured at $37^{\circ} \mathrm{C}$ in a $5 \% \mathrm{CO} 2$ incubator. The following day, fresh basic medium 
was replaced to remove the dead cells and cultured for another 3 to 4 days. After reaching near confluency, the cells were trypsinized and seeded for RNA and protein. Passages were limited to a maximum of three. Isolated MEFs were cultured and set up for adipocyte differentiation assays. Cells were seeded at confluence and grown for two days to achieve growth arrest. Cells were induced to differentiate with adipocyte differentiation medium (Cell Applications, catalog 811D-250) and harvested for protein on day 8 when $>90 \%$ of the cells were differentiated.

\subsubsection{Preadipocyte Isolation and Differentiation Assay}

Visceral and subcutaneous preadipocytes were isolated from visceral and subcutaneous fat pads from wild type mice on postnatal days 12-16. Fat pads were digested in $0.2 \mathrm{mg} / \mathrm{mL}$ collagenase type II (catalog 17101015) in KRBH buffer containing $4 \%$ fatty acid-free BSA while shaking for $20-30$ minutes at $120 \mathrm{RPM}$ at $37^{\circ} \mathrm{C}$. Cells from the digested fat pads were filtered at 100 -microns and centrifuged at $800 \mathrm{~g}$ for 10 minutes at room temperature. $1 \mathrm{~mL}$ of red blood cell (RBC) lysis buffer was added to the cell pellet for 1-3 minutes at room temperature and diluted in PBS. Cells were filtered at 70-microns, centrifuged, and resuspended in 1X DMEM media supplemented with 10\% FBS (Hyclone), sodium pyruvate (1 mmol/L), 1\% Penicillin/Streptomycin (100X), and L-glutamine (2 mmol/L).

$200 \mathrm{~K}$ cells were seeded in 12 well plates and incubated for two days to achieve confluence and to attain contact inhibition. Cells were then induced with $10 \%$ FBS media containing induction cocktail (1uM dexamethasone, $0.5 \mathrm{mM}$ isobutyl methylxanthine, 10 $\mathrm{ug} / \mathrm{ml}$ insulin and 1nM T3 with or without treatment (see below) for the first three days. Maintenance media (differentiation media) was added with $10 \mathrm{ug} / \mathrm{mL}$ of insulin $+1 \mathrm{nM}$ T3 with or without $40 \mathrm{ng} / \mathrm{mL}$ of activin ( $\mathrm{R}$ and $\mathrm{D}$, catalog 338-AC-010), 5uM SB431542 (Millipore, catalog 6164615MG), and 3uM SIS3 (Tocris Bioscience, catalog 52-911-0) mentioned in chapter 4 . Fresh Maintenance media was added every two days with $10 \mathrm{ug} / \mathrm{mL}$ of insulin and $1 \mathrm{nM}$ T3 until harvest. Differentiation efficiency was evaluated by the morphological appearance of differentiated preadipocytes and by staining with Oil Red O and BODIPY on day 8.

\subsubsection{Immunofluorescence}

MEFs differentiated in chamber slides were harvested on day 8 and fixed with $4 \%$ paraformaldehyde for 15 minutes at RT followed by washing with 1X PBS (pH7.4). Cells were permeabilized with $0.2 \%$ triton X-100 in 1X PBS (pH7.4) for 10 minutes followed by washing with 1X PBS (pH7.4) twice. Cells were blocked for 5 minutes in 3\% fetal bovine serum (FBS) diluted in $0.1 \%$ triton X-100 in 1X PBS (pH7.4) and incubated with primary antibody UCP1 (Abcam ab10983) at 1:200 dilution overnight at $4^{\circ} \mathrm{C}$. Next day, cells were washed with $0.1 \%$ triton X-100 in 1X PBS (pH7.4) three times for 15 minutes each and incubated with secondary antibody conjugated with Alexa 568 fluorochrome diluted (1:500) in 3\% FBS 
with $1 \mu \mathrm{l} / \mathrm{ml}$ DAPI and incubated for 1 hour at RT. Cells were washed thrice followed by mounting the cells with mounting medium (Vector laboratories, Prolog Gold).

\subsubsection{Proliferation Assay}

Visceral and subcutaneous preadipocytes were seeded in a 96 well plate. After overnight settling, next day morning cells were treated with vehicle (DMSO) or Activin A or SB431542 or SIS3 and incubated for $48 \mathrm{hr}$. Cells were treated every $24 \mathrm{hrs}$ and total two dose of treatment were given. Quantification of cell proliferation assay was performed using MTT based cell proliferation kit (Sigma 11465007001). Cells were also counted to ascertain the results. The assay was conducted according to the manufacturer protocol to ascertain the proliferation of wild type mouse preadipocytes.

\subsubsection{Flow Cytometry}

Preadipocytes were seeded in a 12 well plate. After overnight settling, next day morning cells were treated with indicated concentration of vehicle (DMSO) or Activin A or SB431542 or SIS3 and incubated for $48 \mathrm{hr}$ similar to proliferation studies. At the end of the experiment, cells were harvested by trypsinizing, resuspending in 10\% FBS media followed by centrifugation at 1000 RPM for 5 minutes. Cells were washed with cold PBS at $500 \mathrm{~g}$ for 3 minutes twice and resuspended the cells in 100uL of $1 \mathrm{x}$ annexin-binding buffer. Added 5uL of FITC annexin $\mathrm{V}$ and $1 \mathrm{uL}$ of $100 \mathrm{ug} / \mathrm{mL}$ propidium iodide (PI). Cells were incubated at room temperature for 15 minutes. Added $400 \mathrm{uL}$ of $1 \mathrm{X}$ annexin binding buffer after incubation and mixed gently and stored the sample on ice until stained the samples by flow cytometry. Hydrogen peroxide was used as a positive control. Negative control had no inducing agent.

\subsubsection{RNA-Seq Library Prep}

Total RNA was isolated from undifferentiated and differentiated mouse embryonic fibroblast cells (MEFs) using the trizol method (Invitrogen). Submitted RNA samples from differentiated groups was subjected to analysis on an Agilent Bioanalyzer and spectrophotometric analysis to determine RNA quality. After passing this initial screening 500ng of total RNA will be enriched for polyA RNA or depleted of rRNA then used to prepare libraries for sequencing using the NEB Next Directional RNA Library Prep Kit for Illumina. Libraries amplified for 9-15 cycles, depending on original starting material, as the final step of library preparation. Before sequencing, small aliquots of this material will be quantified by qPCR utilizing the KAPA Library Quantification kit. Quantification data from qPCR are used to balance the barcodes for final pooling before sequencing. Following this final pooling the library pools are sized to a target size of 300bp on a Pippin Prep instrument. The sized libraries are examined on an Agilent High Sensitivity DNA chip, quantified using real-time PCR, and used for sequencing on the Illumina NextSeq 500 at UTHSC core. 


\subsubsection{RNA-Seq Processing, Sequencing, and Analysis of RNA-Seq Data}

All fastq files were gathered from the sequencer, and quality assurance was performed using FASTQC. Reads were trimmed to remove any nucleotide with a PHRED score $<$ Q20, and the trimmed FASTQ files were aligned to the Genome Reference Consortium Mouse Build $38 \mathrm{~mm} 10$ mouse fasta reference library using RNA STAR. Once aligned, the SAM files were collected and mined for the read count information of each gene present in the reference file. 30-40 million reads were recorded per sample. Read counts were normalized using Median Ratio normalization method and across the entire experiment. Principle component analysis and Pearson's coefficient plots were performed on the normalized transcriptome profile. Differential gene expression between wildtype and double knockout activin cells was accessed using false discovery rate $($ FDR $)<0.05$ and fold change $>1.5$. cutoff. A Wilcoxon's t test was used to determine significance between conditions, and all genes that failed to yield a $p$-value greater than 0.05 were removed. Benjamini and Hochberg false discovery rate was performed on the trimmed gene list, and all genes failing to yield a false discovery rate of less than 0.05 were discarded. Heatmaps and scatter plot were generated using R script. Heat map for browning genes that were upregulated in activin $\mathrm{dKO}$ were plotted using Morpheus software. Pathway analysis was performed using iPathway Guide. EnrichR was used to analyze cells specific pathways.

\subsubsection{Glycerol Estimation}

Glycerol estimation was performed according to the manufacturer protocol (Sigma F6428, G7793) to ascertain the bioactivity of isoproterenol on differentiated adipocytes (MEFs and 3T3-L1) after treatment with ISO. US pharmacopeia reference standard (Sigma 1351005) or generic version of isoproterenol (Sigma I6504)] was used with different conditions (freshly prepared or frozen stocks) to eliminate the bias of its bioactivity due to quality or storage.

\subsubsection{Microscopy}

Images for all in vivo and in vitro experiments were taken using EVOS FL Auto Imaging System (Thermo Fisher, Foster City, CA). Green, Red and DAPI filters were used for tracking the lipid and nucleic acid respectively in differentiation experiments. Image J software was used to quantitate the protein in some experiments.

\subsection{Statistical Analysis}

GraphPad Prism version 9.0 (GraphPad Software, San Diego, CA) was used for statistical analysis for each experiment unless otherwise mentioned. Mouse weights study data and GTT are expressed as mean \pm standard error of the mean (SEM). A linear mixed model two-way ANOVA for body weight (grams) was used to account for repeated measures and to examine differences in weights among genotypes over time. The Šídák test was used to control for the multiple comparisons. mRNA levels relative fold changes were calculated 
using the Relative Expression Software Tool 2009 (REST) (Qiagen, Hilden, Germany and Technical University of Munich, Germany), excel and Graph pad prism. For the remaining studies, the Mann-Whitney test was used to compare MRI data, such as fat mass and lean mass, between genotypes. A p value $<0.05$ was considered statistically significant for each experiment. Note: ${ }^{*} \mathrm{p}<0.05,{ }^{* *} \mathrm{p}<0.005,{ }^{* * * *} \mathrm{p}<0.00005$. 


\section{Chapter 4}

\section{SMAD2/3 Differentially Regulate Adiposity and the Growth of Subcutaneous White Adipose Tissue ${ }^{1}$}

\subsection{Introduction}

Obesity and the associated metabolic syndrome are major medical problems in the United States (Chooi, Ding, and Magkos, 2019). Obesity occurs due to an unhealthy increase in white adipose tissue (WAT) (P. Wang et al., 2008; Jo et al., 2009). Adipose tissue plays a crucial role in maintaining energy homeostasis. The sustained expansion of WAT dysregulates energy homeostasis by affecting production of adipocytokines such as leptin, adiponectin and resistin (Gardan, Gondret, and Louveau, 2006; Rabe et al., 2008; Bederman et al., 2018). Dysregulation of adipocytokine production and release impacts satiety and is a major determinant of obesity (Unger et al., 2010). Adipose tissue dysfunction occurs with unhealthy enlargement of adipocytes, associated with inflammation and insulin resistance (J. M. Rutkowski, J. H. Stern, and P. E. Scherer, 2015; Choe et al., 2016; Longo et al., 2019). Disproportionate contributions from visceral and subcutaneous WAT depots are well-described for their important roles in obesity and its co-morbidities (Gesta, Y. H. Tseng, and Kahn, 2007).

TGF- $\beta$ and several other ligands in this family including activin A and activin B, have direct effects on growth and development of a variety of tissues, including adipose, thereby impacting energy homeostasis (C. W. Brown, Houston-Hawkins, et al., 2000; Choy, Skillington, and Derynck, 2000; L. Li et al., 2009; C. K. Tan, Leuenberger, et al., 2011; Yadav, Quijano, et al., 2011; Bonomi et al., 2012; M. J. Lee, 2018). Activins A and B have pleiotropic effects, impacting preadipocyte proliferation, differentiation, and determining cell fate (C. W. Brown, L. Li, et al., 2003; Hirai et al., 2005; Zaragosi et al., 2010; Zamani and Chester W. Brown, 2011; Xue et al., 2016; Q. Zhu et al., 2018). Activins A and B signal through activin receptors and downstream transcriptional transducers, SMAD2 and SMAD3. In turn, SMAD2 and SMAD3 regulate the transcription of downstream adipogenic genes

\footnotetext{
${ }^{1}$ Modified: From final submission with permission from John Wiley E Sons, Inc. (Kumari et al., 2021). "SMAD2 and SMAD3 differentially regulate adiposity and the growth of subcutaneous white adipose tissue". In:FASEB journal: official publication of the Federation of American Societies for Experimental Biology.
} 
(Darlington, Ross, and O. A. MacDougald, 1998; C. W. Brown, L. Li, et al., 2003; Fei et al., 2010). During adipocyte differentiation the activin signaling pathway phosphorylates SMAD2/SMAD3, thereby inhibiting downstream transcriptional regulators of adipogenesis, $\mathrm{C} / \mathrm{EBP} \alpha, \mathrm{C} / \mathrm{EBP} \beta, \mathrm{C} / \mathrm{EBP} \delta$ and PPAR $\gamma$ (Darlington, Ross, and O. A. MacDougald, 1998; Choy, Skillington, and Derynck, 2000; Zaragosi et al., 2010; C. K. Tan, Leuenberger, et al., 2011; Zamani and Chester W. Brown, 2011; C. K. Tan, Chong, et al., 2012).

The functions of activin signaling are well studied in many tissues, including adipose (K. Tsuchida et al., 2004; Lonardo et al., 2011; Dani, 2013; Latres et al., 2017; Song et al., 2017) 2017, Song, Owusu-Ansah et al. 2017). In previous studies, we have reported that activin deficiency reduces adiposity and has beneficial effects on energy metabolism (C. W. Brown, L. Li, et al., 2003; L. Li et al., 2009). Activin A inhibits differentiation via SMAD2 signaling and promotes self-renewal of human adipose progenitors by inducing expression of Nanog (Q. Zhu et al., 2018). Although activin A inhibits differentiation in 3T3-L1 pre-adipocytes and in human adipocyte progenitors (Hirai et al., 2005; Zaragosi et al., 2010; M. J. Lee, 2018), little is known about the role of activins on the differentiation of preadipocytes derived from mouse adipose depots. SMAD2 and SMAD3, the downstream mediators of activin signaling, regulate many biological processes including embryogenesis, adipose tissue growth, adipocyte differentiation, and cell fate decisions (Nomura and E. Li, 1998; Weinstein et al., 1998; Datto et al., 1999; C. K. Tan, Leuenberger, et al., 2011; Tsurutani et al., 2011; Yadav, Quijano, et al., 2011).

SMAD2 and SMAD3 are closely related proteins, sharing $92 \%$ amino acid similarity. These proteins form homodimers or heterodimers to form functional complexes with SMAD4 (Aragon et al., 2019). Although there is functional overlap, Smad2 and Smad3 also have distinct functions such as the critical role of Smad2 during early gastrulation (Nomura and E. Li, 1998; Waldrip et al., 1998; Weinstein et al., 1998; Heyer et al., 1999; Ju et al., 2006; Kriseman et al., 2019). In contrast, Smad3 global knockout mice are viable and fertile. However, Smad3 mice undergo postnatal growth retardation with immunodeficiencies and shortened lifespan (Y. Zhu et al., 1998; Ashcroft et al., 1999; Datto et al., 1999; Yang et al., 1999; Q. Li, Pangas, et al., 2008; Tsurutani et al., 2011). Previous studies have demonstrated that the global knockout of Smad3 protects from diet-induced obesity (DIO) with favorable metabolic effects, including improved glucose tolerance and insulin sensitivity (C. K. Tan, Leuenberger, et al., 2011; Tsurutani et al., 2011; Yadav, Quijano, et al., 2011; Yadav and Rane, 2012). However, these studies could not exclude indirect, secondary influences from other metabolically active tissues such as skeletal muscle, pancreas, and liver, as shown by the tissue-selective effects of Smad3 deficiency on hepatic gluconeogenesis and pancreatic beta cell apoptosis (Yadav, Devalaraja, et al., 2017; J. H. Lee et al., 2020).

Previous studies have demonstrated roles for activin A, activin B and SMAD3 on body composition using a variety of activin and Smad3 alleles (C. W. Brown, HoustonHawkins, et al., 2000; C. W. Brown, L. Li, et al., 2003; L. Li et al., 2009; Bonomi et al., 2012). However, due to embryonic lethality in Smad2 global knockout mice, SMAD2's effect on 
adiposity has not been determined. The adipose-selective roles of SMAD3 have also not been assessed. Accordingly, we investigated the adipose-selective effects of Smad2 and Smad3 deficiency on adipose tissue development and function.

\subsection{Results}

\subsubsection{SMAD2 and SMAD3 Proteins Exhibit Different Temporal Expression Du- ring Adipose Tissue Development}

Global Smad3 deletion impacts growth, as mice are smaller at weaning (Datto et al., 1999; C. K. Tan, Leuenberger, et al., 2011). Preadipocyte proliferation and differentiation begins at e14.5 in the subcutaneous WAT anlage and approximately 4 days postnatally in visceral WAT (Sarjeant and Stephens, 2012; Traustadottir et al., 2013). Therefore, we looked at the total level of SMAD2 and SMAD3 proteins and their phosphorylated forms in both visceral and subcutaneous WAT from wild type (WT) mice, at 4 time points ranging from postnatal weeks 1-14 (Figure $4.1 \mathrm{~A}-\mathrm{B})$. SMAD2 protein and its phosphorylated form (pSMAD2) remained unchanged in both visceral and subcutaneous WAT over 14 weeks. In contrast, SMAD3 protein and its phosphorylated form (pSMAD3) displayed temporal changes in expression, highly expressed at 1 and 3 weeks but later declining in both visceral and subcutaneous WAT. This suggests that SMAD3 may contribute to early adipose tissue development, whereas SMAD2 may contribute additionally to later stages of development and mature adipocyte function.

We also examined the protein levels of SMAD2 and SMAD3 in WT MEFs induced to differentiate into adipocytes, in vitro (Figure 4.2 A). While normalized SMAD2 protein levels did not significantly change, SMAD3 was highly expressed in undifferentiated MEFs as well as in cells at day 3 post-induction, but substantially declined by day 5 , the time at which most of the cells had assumed the characteristics of mature adipocytes. However, pSMAD2 and pSMAD3 protein levels remained unchanged. Collectively, these findings are consistent with SMAD3 potentially contributing to the early growth phase of white adipose tissues and to the early stages of adipocyte differentiation. To determine whether the protein levels corresponded to changes at the transcriptional level, we measured Smad2 and Smad3 mRNA. In contrast to the patterns observed at the protein level, both transcripts increased from day 3 to day 8 during the differentiation of WT MEFs, visceral, and subcutaneous preadipocytes in vitro (Figure 4.2 B-C). Collectively, these $\mathrm{mRNA}$ and protein studies are consistent with SMAD2 and SMAD3 being regulated at the transcriptional, post-transcriptional and posttranslational levels during adipogenesis.

\subsubsection{Activins Regulate Phosphorylation of SMAD2 and SMAD3 in Adipogenic Cells}

SMAD2 and SMAD3 are well-established downstream mediators of activin signaling, regulated by their phosphorylation in the presence of activins[19, 23]. Activin-dependent 


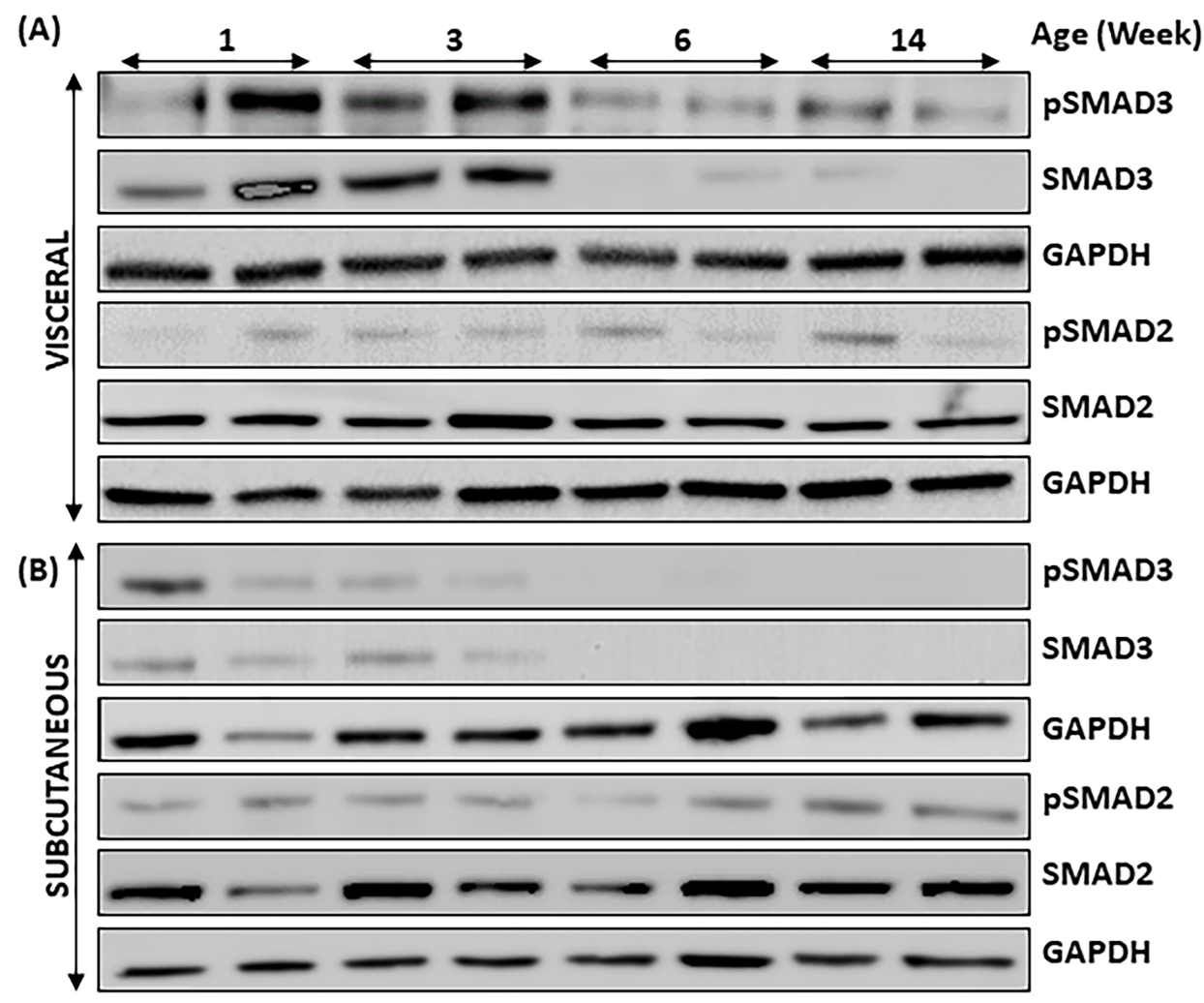

Figure 4.1: SMAD2 and SMAD3 Expression in White Adipose Tissues. (A) Western blot showing temporal changes in protein levels of pSMADs and SMADs in visceral WAT and (B) in subcutaneous WAT. Note: reduced expression over time for pSMAD3 and SMAD3 in both depots while no change in pSMAD2 and SMAD2. 
(A)

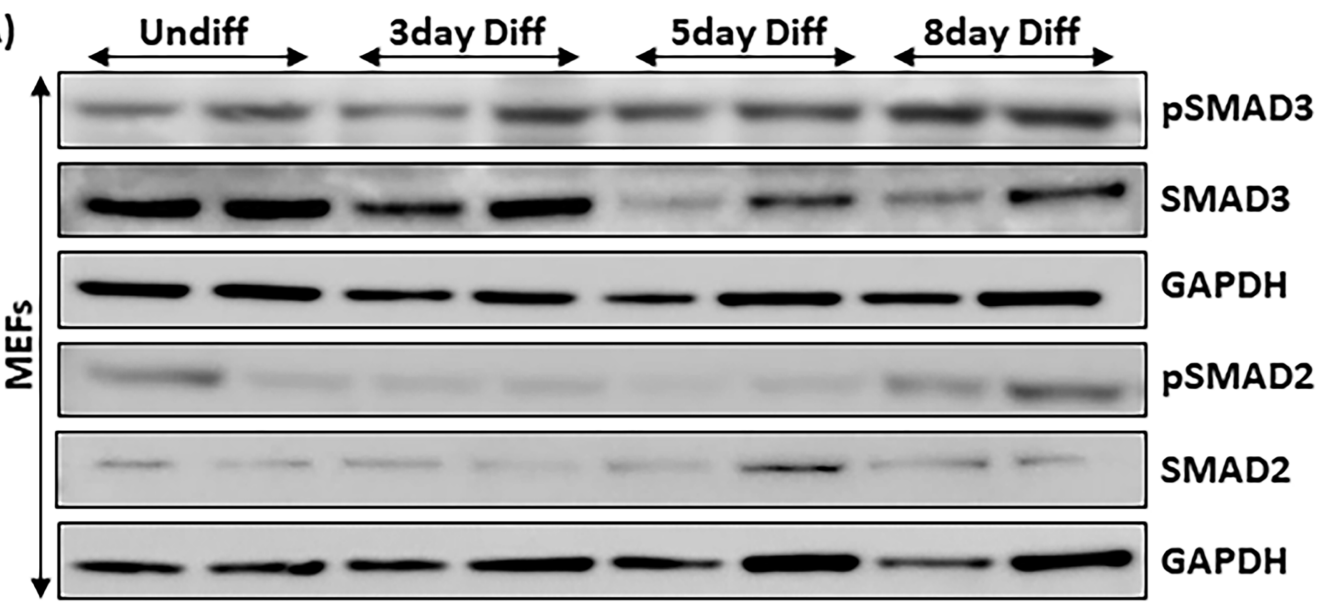

(B)
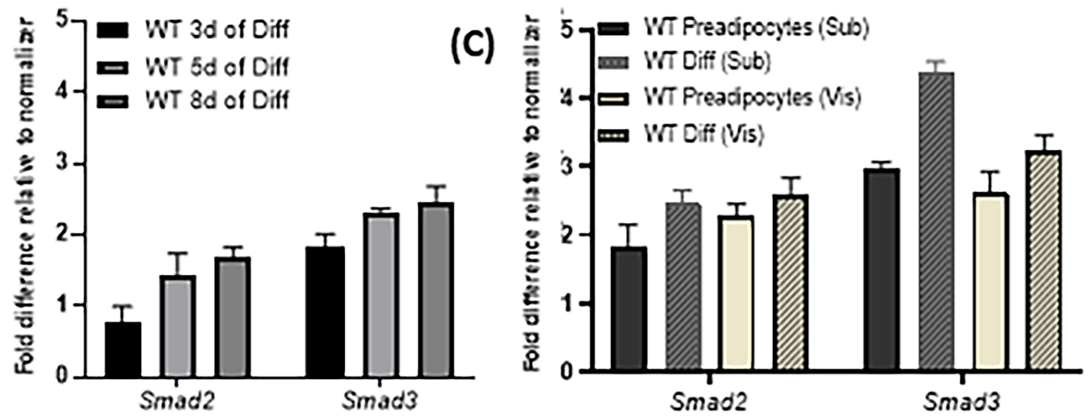

Figure 4.2: SMAD2 and SMAD3 Expression in Differentiating MEF-Derived Adipocytes. (A) High protein level of SMAD3 in undifferentiated MEFs and on differentiation day3, declining by day5 and day8. No change in protein levels of SMAD3, pSMAD2 or SMAD2 ( $n=2$, biological replicates). GAPDH was used as a loading control. (B) Smad2 and mRNA levels during the differentiation of MEFs and (C) Smad2 and Smad3 mRNA levels from visceral and subcutaneous preadipocytes and differentiated adipocytes isolated from wild type mice. Diff; Differentiated, Undiff; undifferentiated, Sub; subcutaneous, Vis; visceral. 


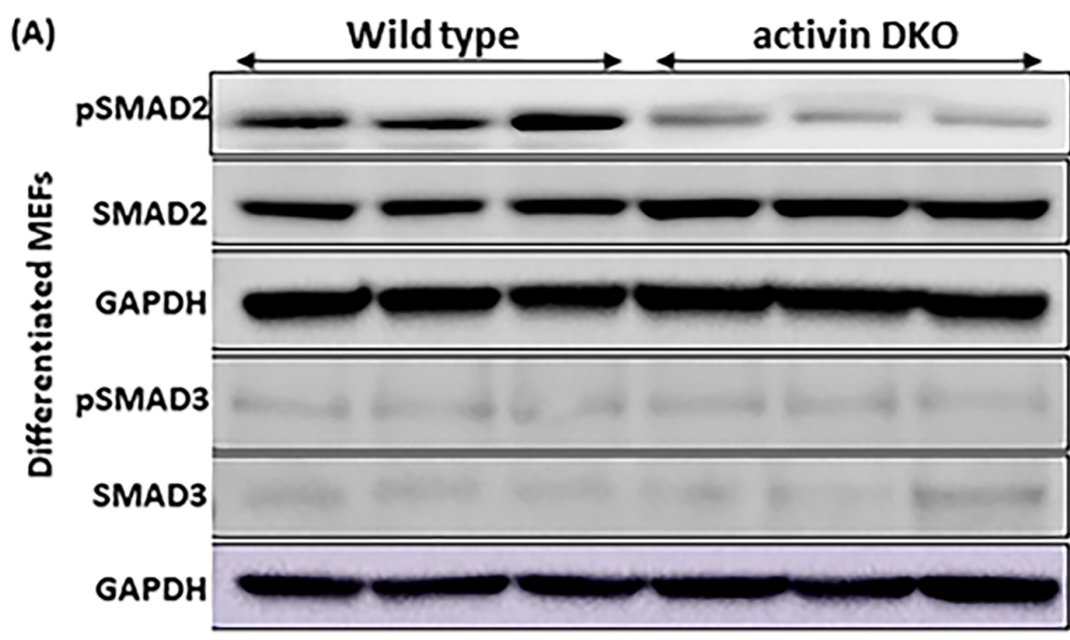

(B)

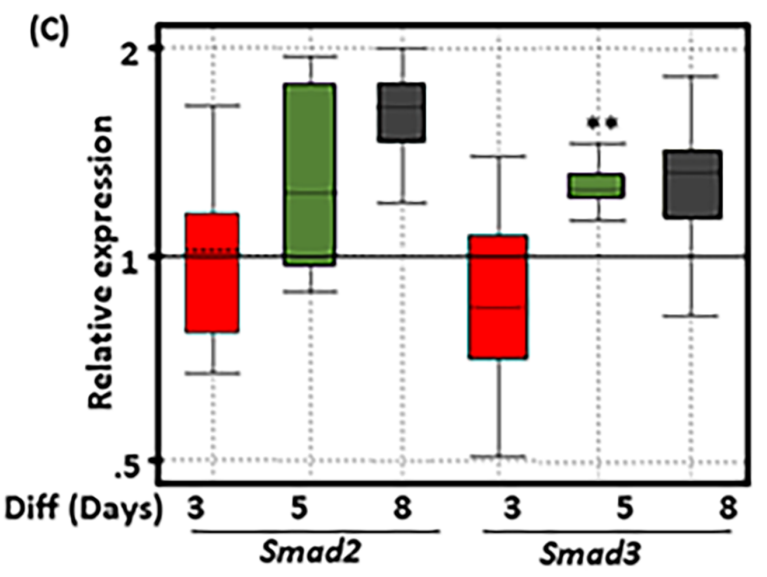

Figure 4.3: Effect of Activin Deficiency on SMAD Expression and Phosphorylation. (A) Western blot of pSMAD2, pSMAD3, SMAD2 and SMAD3 levels in WT and activin-deficient, fully differentiated MEFs ( $n=3$ biological replicates). Significantly reduced pSMAD2 levels in activin deficient adipocytes. GAPDH was used as a loading control. (B) quantitation of pSMAD2 ( $\mathrm{p}^{*}=0.012$ ) normalized to SMAD2 from (A). (C) quantitation of Smad2 and Smad3 mRNA from differentiating, activin-deficient MEFs. ( $\mathrm{p}^{* *} \leq$ 0.005)The data are presented as ratios of activin-deficient MEFs mRNA levels to WT set at 1 (solid, horizontal bar). Whisker box plots with boxes representing the middle $50 \%$ of observations and whisker bars representing the maximum and minimum values. Diff; Differentiation. 
phosphorylation of SMAD2 has been demonstrated, but it remains unclear whether activin also regulates SMAD3 phosphorylation in adipocytes. Accordingly, we examined the phosphorylation of SMAD2 and SMAD3 using MEFs isolated from activin double knockout (DKO) mice with global knockout of activin A (Inhba-/-) and activin B (Inhbb-/-). The activin DKO MEFs were differentiated into adipocytes and the SMAD2 and SMAD3 phosphorylation status was examined on day 8. Activin DKO adipocytes exhibited significantly less SMAD2 phosphorylation than WT but showed no change in the phosphorylation of SMAD3 (Figure 4.3 A-B). Like other cell types, activin A treatment of WT preadipocytes isolated from subcutaneous fat robustly induced SMAD2 and SMAD3 phosphorylation (data not shown).

Additionally, we assessed MEFs isolated from activin DKO mice for Smad2 and Smad3 mRNA levels during differentiation. mRNA levels of Smad2 and Smad3 trended higher at days 5 and 8 relative to WT, with a significantly higher level of Smad3 on day 5 (Figure $4.3 \mathrm{C}$ ). These data suggest that in addition to the effects of MEF differentiation on Smad2 and Smad3 mRNA levels, activins may also play a role.

\subsubsection{Subcutaneous and Visceral Preadipocytes Differ in Adipogenic Efficiency Influenced by Activin Signaling}

Activin A inhibits differentiation of 3T3-L1 cells and human-adipocyte progenitors (Hirai et al., 2005; Zaragosi et al., 2010; Aykul et al., 2020); however, whether depot-selective effects of activins on adipogenesis occur in preadipocytes is unknown. Thus, we examined the adipogenic efficiency of primary preadipocytes isolated from subcutaneous and visceral WAT, and the depot-selective effects of activin A and its inhibitors. Subcutaneous and visceral preadipocytes from WT mice were induced to differentiate with or without activin A, activin receptor inhibitor, SB431542, or SMAD3-specific inhibitor, SIS3 (Jinnin, Ihn, and Tamaki, 2006). We assessed differentiation by BODIPY and Oil Red O staining on day 8 of differentiation. While differentiation efficiency was approximately $90 \%$ in preadipocytes isolated from the subcutaneous depot (Figure $4.4 \mathbf{A - \mathbf { B } ^ { \prime }}$ ), preadipocytes from the visceral depot differentiated poorly, exhibiting 20-30\% differentiation efficiency (Figure 4.5 A-B' $\mathbf{B}^{\prime}$ ). Activin A treatment reduced the differentiation efficiency of subcutaneous preadipocytes, and activin A-treated visceral preadipocytes differentiated less efficiently than untreated controls, as very few cells with multilocular lipid droplets were observed (Figure 4.44.5). Although it is clear that activin A treatment in primary subcutaneous preadipocytes diminished differentiation, this was not as clear in visceral preadipocytes, and was more challenging to interpret the magnitude of the effect in the context of a cell population that already differentiated poorly at baseline.

In contrast to activin A, the activin-like kinase (ALK) inhibitor SB431542, that blocks type I activin receptors (ALK4, ALK7) and type I TGF- $\beta$ receptor (ALK5), promoted lipid accumulation in cells derived from both depots, although less so in cells from the visceral depot. However, treatment with SB431542 greatly enhanced the differentiation of visceral 
(A)
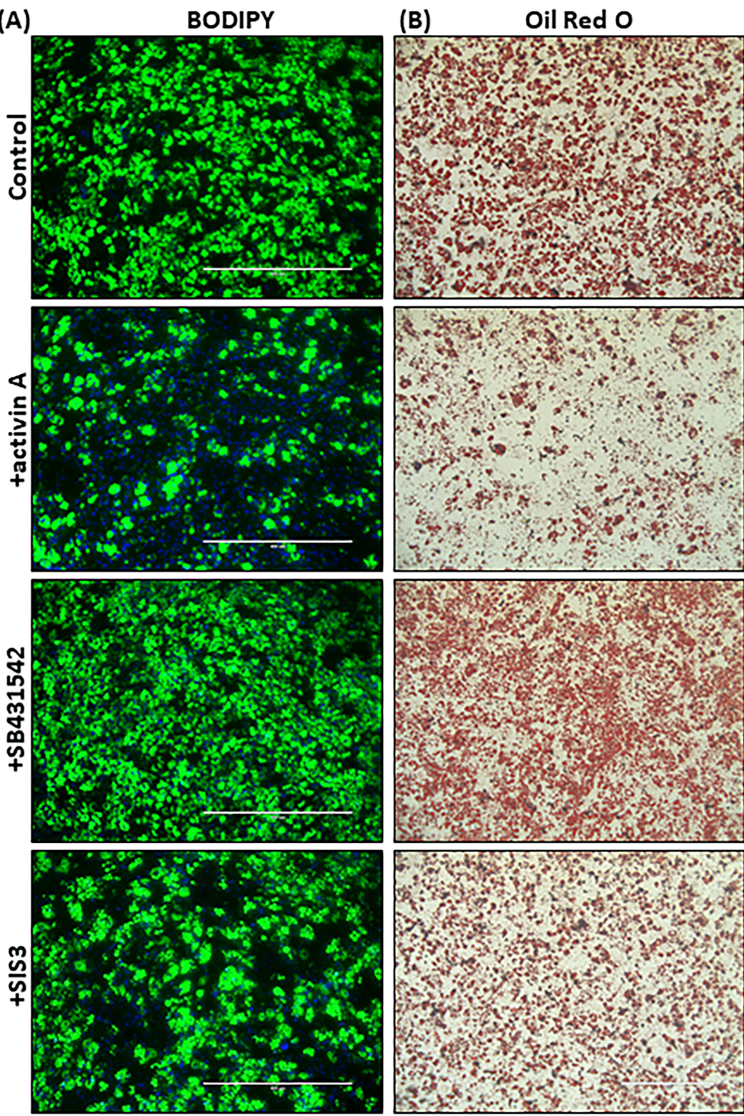

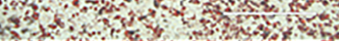

(C)

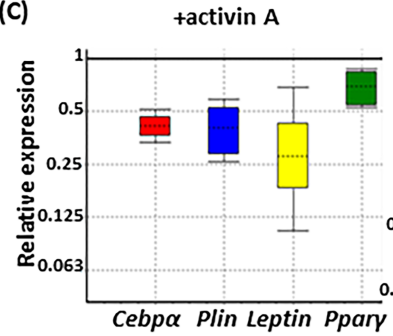

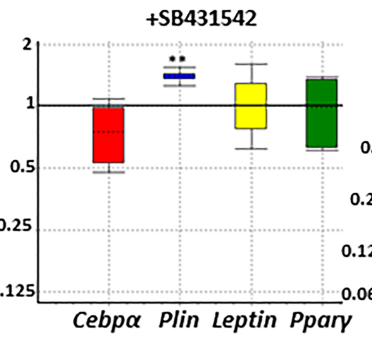

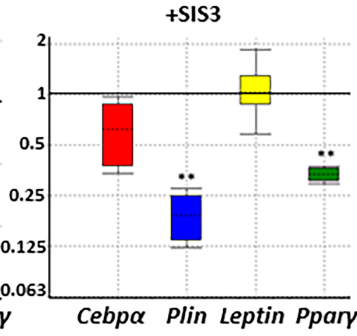

Figure 4.4: Activin A, SB431542 and SIS3 Regulate Differentiation of Subcutaneous Preadipocytes. (A) BODIPY staining on day 8 of differentiation (green, counterstained with DAPI (blue)), (B) Oil Red O and $\left(B^{\prime}\right)$ Oil Red O, full well view. Activin A (40ng/mL) showing reduced adipocyte differentiation, while SB431542 $(5 \mu \mathrm{M})$ enhanced differentiation.

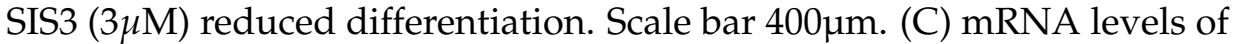
adipogenic markers Cebp $\alpha$, Plin, Leptin and Ppar $\gamma$ on day $8\left({ }^{*} \mathrm{p}<0.05,{ }^{* *} \mathrm{p}<\right.$ 0.005). 

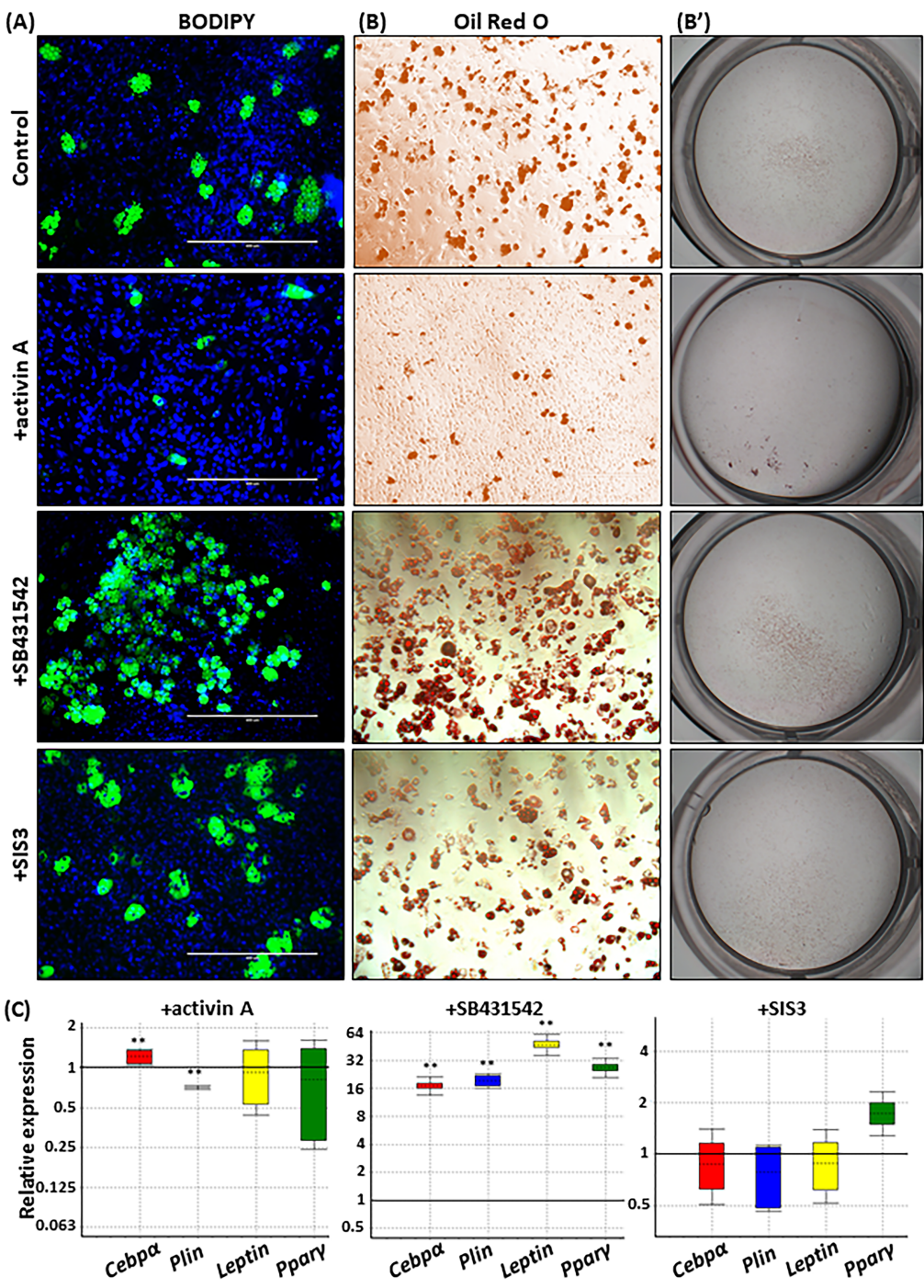

Figure 4.5: Activin A, SB431542 and SIS3 Regulate Differentiation of Visceral Preadipocytes. (A) BODIPY staining (green counter stained with DAPI blue), (B) Oil Red $\mathrm{O}$ and (B') Oil Red O staining on day 8 of differentiation. Activin A $(40 \mathrm{ng} / \mathrm{mL})$ treated cells failed to differentiate while SB431542 $(5 \mu \mathrm{M})$ markedly induced differentiation. SIS3 $(3 \mu \mathrm{M})$ treatment had no impact on differentiation. Scale bar $400 \mu \mathrm{m}$. (C) mRNA levels of Cebp $\alpha$, Plin, Leptin, Ppar $\gamma$ on day 8 for the indicated treatments (*p $\left.<0.05,{ }^{* *} \mathrm{p}<0.005\right)$. 
cells at the cellular and molecular level, with a less robust effect in subcutaneous cells, which already achieved 90\% differentiation efficiency at baseline. In contrast, SMAD3 inhibitor, SIS3, reduced differentiation of subcutaneous, but not visceral preadipocytes (Figure 4.5 A-B' ${ }^{\prime}$ ). Thus, SMAD3 inhibitor showed a robust inhibitory effect in subcutaneous preadipocytes at the cellular and molecular level, but not as clearly in visceral, although the relatively poor differentiation of visceral cells again makes interpretation challenging. Overall, these data suggest that subcutaneous and visceral preadipocytes differ in their differentiation potential and respond differently to processes that impact activin signaling during adipogenesis.

To further assess the impact of activin A signaling on adipogenesis, we examined the expression of adipocyte-specific markers in fully differentiated subcutaneous preadipocytes receiving activin A, SB431542 or SIS3 (Figure 4.4 C). Activin A treated cells showed reduced expression of adipogenesis and mature adipocyte markers, Cebp $\alpha$, Perilipin, Leptin and Ppary, in keeping with the reduced differentiation observed microscopically. In contrast, SB431542 did not impact Cebp $\alpha$, Ppar $\gamma$ and Leptin, but Perilipin, a lipid droplet marker, was

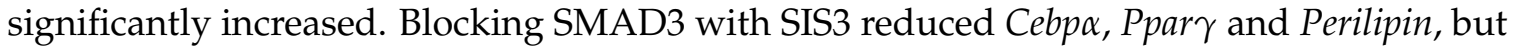
not Leptin. These findings are consistent with roles for activin signaling during adipogenesis, impacting the expression of key adipogenic regulators.

For visceral preadipocytes, activin A treatment modestly increased the expression of Cebp $\alpha$ relative to control cells, while Perilipin expression was significantly reduced (Figure 4.5 C). Leptin and Ppary expression did not change. Activin receptor inhibitor, SB431542, markedly increased Cebp $\alpha$, Perilipin, Leptin and Ppar $\gamma$ transcript levels, while SMAD3 inhibitor, SIS3, did not significantly impact mRNA levels. Collectively, these findings suggest that subcutaneous and visceral preadipocytes may differ in their responses to activin signaling during adipogenesis with much higher differentiation capacity in subcutaneous

preadipocytes. Further, activin signaling and its downstream effector, SMAD3, may contribute to preadipocyte differentiation in primary culture cells, impacting the expression of key adipogenic regulators.

\subsubsection{Adipose-Selective Deletion of Smad2 or Smad3 Have Different Effects on Weight Gain Under High-Fat Diet Conditions}

Smad2 global knockout mice die in utero, while Smad3 knockout mice are smaller at weaning (Nomura and E. Li, 1998; Waldrip et al., 1998; Weinstein et al., 1998; Y. Zhu et al., 1998; Datto et al., 1999; Yang et al., 1999). Ubiquitous knockout of Smad3 protects mice from diet-induced obesity (Datto et al., 1999; C. K. Tan, Leuenberger, et al., 2011; Tsurutani et al., 2011; Yadav, Quijano, et al., 2011). However, it is plausible that loss of Smad3 in non-adipose tissues contributes to this phenotype. To explore the adipose-selective effects of Smad2 and Smad3, we generated Smad2, Smad3 and Smad2/3 conditional knockout mice (hereafter, Smad2cKO and Smad3cKO, respectively) using Adiponectin-Cre (Figure 4.6 A-B). 

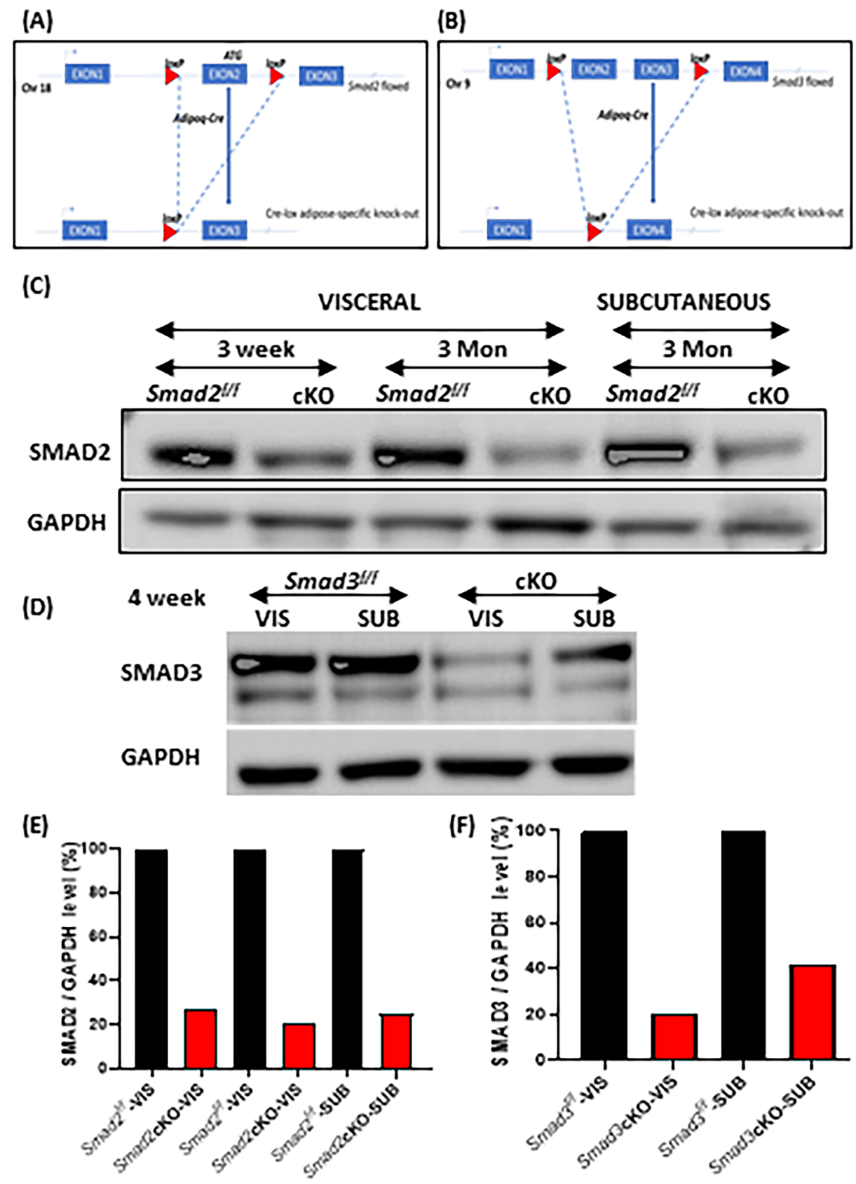

Figure 4.6: Generation of Smad2cKO and Smad3cKO Mice Using Adiponectin-Cre. (A) Deletion strategy for Smad2 and (B) Smad3. (C-D) western blot showing efficiency of Adiponectin-Cre for Smad2 and Smad3, respectively (E) Quantitation of SMAD2 protein and (F) Quantitation of SMAD3 protein normalized to GAPDH in both visceral and subcutaneous WAT. 
Adiponectin-Cre-mediated reduction of SMAD2 and SMAD3 was confirmed by western blot (Figure 4.6 C-F).

In contrast to the global knockout, adipose tissue-selective reduction of Smad2 or Smad3, did not result in obvious growth defects under regular diet conditions, and the mice were viable and fertile. Next, we examined the effects under HFD conditions. Control mice normally remain lean on regular diet, while HFD induces obesity over time. Adipose-selective Smad2 deletion did not have a significant effect on body weight in males, irrespective of diet, while Smad2cKO females tended to be slightly heavier than control mice on HFD at 23 weeks, but no difference was observed on LFD (Figure 4.7 A-B). In contrast, Smad3cKO male mice on HFD displayed divergence in body weight at 8 weeks and continued with lower body weights relative to diet-matched controls until the end of the experiment at 23 weeks. However, the body weight differences did not reach significance (Figure 4.7 C). Smad3cKO female mice displayed no differences in body weight or size relative to control littermates (Figure 4.7 D). Since body weight differences in males were only observed under HFD conditions, we next examined the impact of HFD on SMAD3 expression and phosphorylation in WAT.

Smad3 flox/flox control mice were fed LFD or HFD for 8 weeks or 23 weeks. Visceral and subcutaneous fat depots were examined for pSMAD3 and SMAD3 protein levels by western blot. After 8 weeks on diet, normalized protein levels of pSMAD3 were higher in visceral and subcutaneous WAT depots from HFD fed mice relative to LFD, and at 23 weeks approximated 8-week LFD levels (Figure $4.8 \mathbf{A}-\mathbf{B}, \mathbf{A}^{\prime}-\mathbf{B}^{\prime}$ )). Subcutaneous WAT SMAD3 levels were higher on HFD at 8 weeks and 23 weeks relative to LFD fed mice; however, no changes were observed in SMAD3 levels in visceral WAT. This suggests that SMAD3 protein levels are influenced by diet in an adipose depot-selective fashion.

Smad3cKO mice showed similar fasting blood glucose levels and after glucose injection, we observed slightly lower blood glucose levels in Smad3cKO male mice on HFD relative to control littermates, which was consistent at each time point of glucose tolerance testing (GTT), but the differences were not significant (Figure 4.9 A). Female Smad3cKO mice on HFD showed modestly higher blood glucose levels relative to controls at 15 minutes and 30 minutes but showed no difference at 90 minutes (Figure 4.9 B).

Smad2cKO mice displayed no significant differences from controls throughout the GTT experiment, irrespective of sex and diet conditions (Figure 4.10 A-B). Also, there were no differences in whole body fat mass, lean mass or water composition between controls and Smad2cKO mice, irrespective of diet or sex (Figure $4.10 \mathrm{C}-\mathrm{J}$ ).

\subsubsection{Smad3-Deficient Male Mice Exhibit Differences in Weight Gain and Sub- cutaneous Fat Mass}

Others have shown reduced body weight in Smad3 global knockout mice, corresponding to reduced visceral and subcutaneous fat mass (C. K. Tan, Leuenberger, et al., 2011; Tsurutani 
(A)

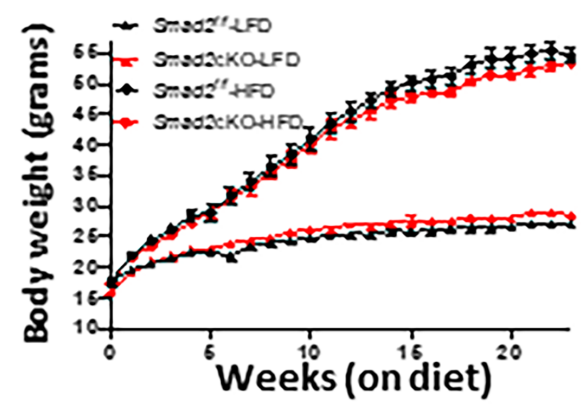

(C)

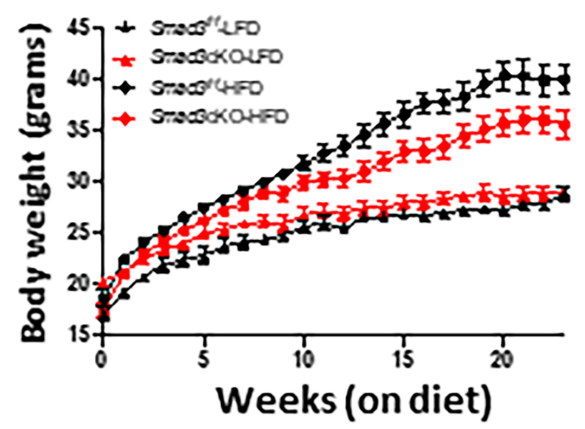

(B)

Female

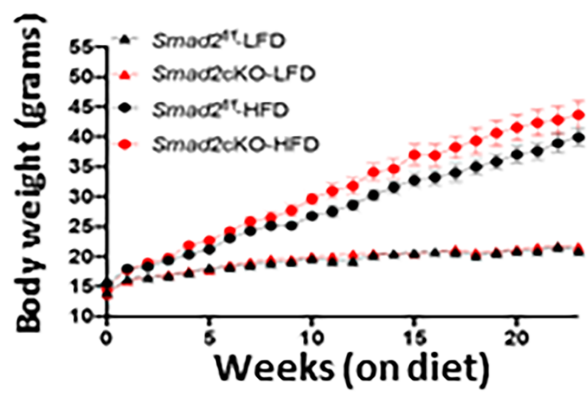

(D) Female

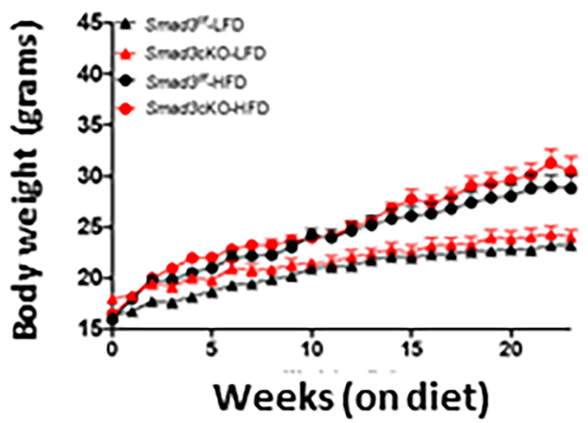

Figure 4.7: Adipocyte-Selective Deletion of Smad3 in Males Results in Lower Body Weight. (A) Growth curves showing no detectable differences in the body weights of Smad2cKO male mice relative to control mice fed HFD or LFD (B) Trend of greater body weights in Smad2cKO females on HFD (C) Smad3cKO male mice exhibited lower body weight on HFD compared to controls (D) No difference in body weight in Smad3cKO female mice on either diet relative to age-matched control. $n=8-11$. 
(A)

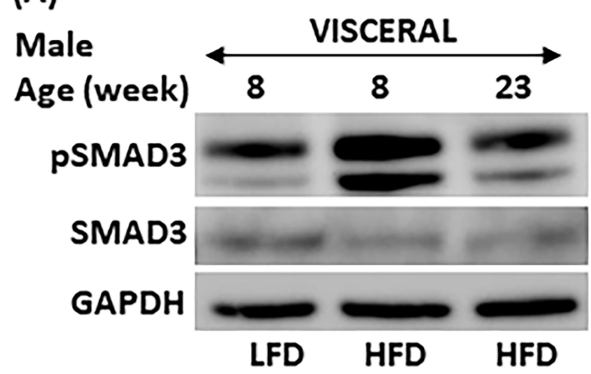

(B)

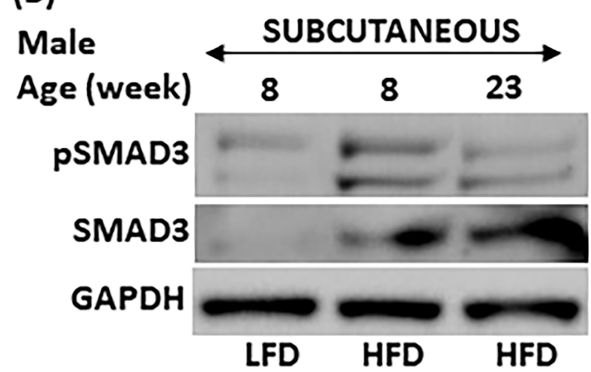

$\left(A^{\prime}\right)$

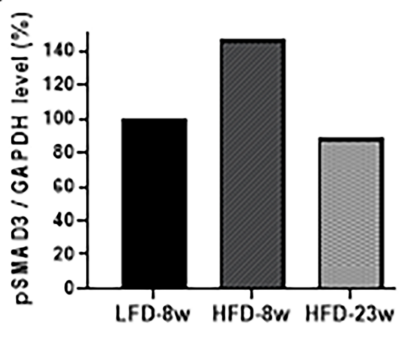

$\left(B^{\prime}\right)$

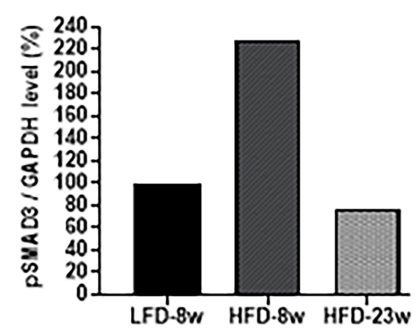

Figure 4.8: Phosphorylation of SMAD3 on LFD or HFD for 8 or 23 weeks. (A-B) Control mice (Smad3fflox/flox $)$ on LFD or HFD for 8 weeks or 23 weeks. At 8 weeks, GAPDH normalized protein levels of pSMAD3 were higher in visceral and subcutaneous depot from HFD fed mice relative to LFD. At 23 weeks pSMAD3 approximated 8-week LFD levels (upper band).

Subcutaneous SMAD3 levels were higher on HFD at 8 weeks and 23 weeks relative to LFD in subcutaneous adipose depot. $\left(\mathrm{A}^{\prime}-\mathrm{B}^{\prime}\right)$ Quantitation of pSMAD3 from visceral and subcutaneous depot respectively.
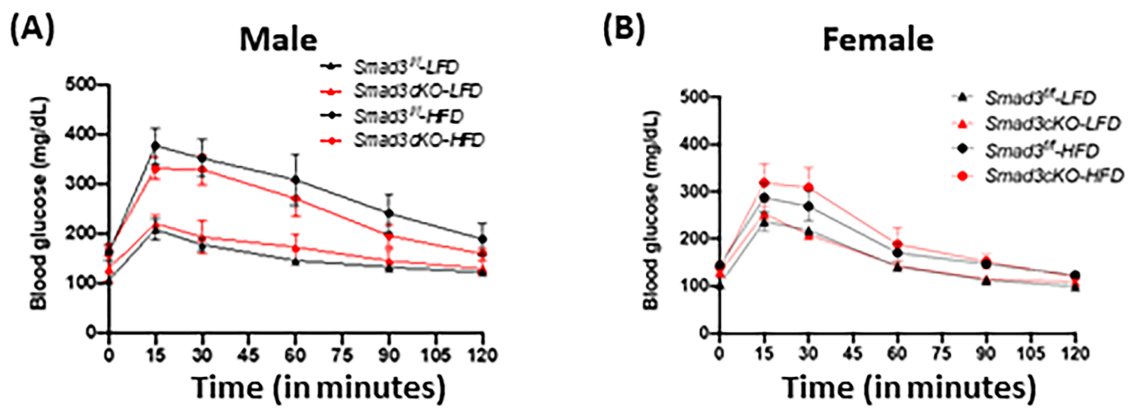

Figure 4.9: Glucose Tolerance Test. (A-B) GTT of (Smad3fflox/flox $)$ control and Smad3cKO mice on LFD and HFD. 

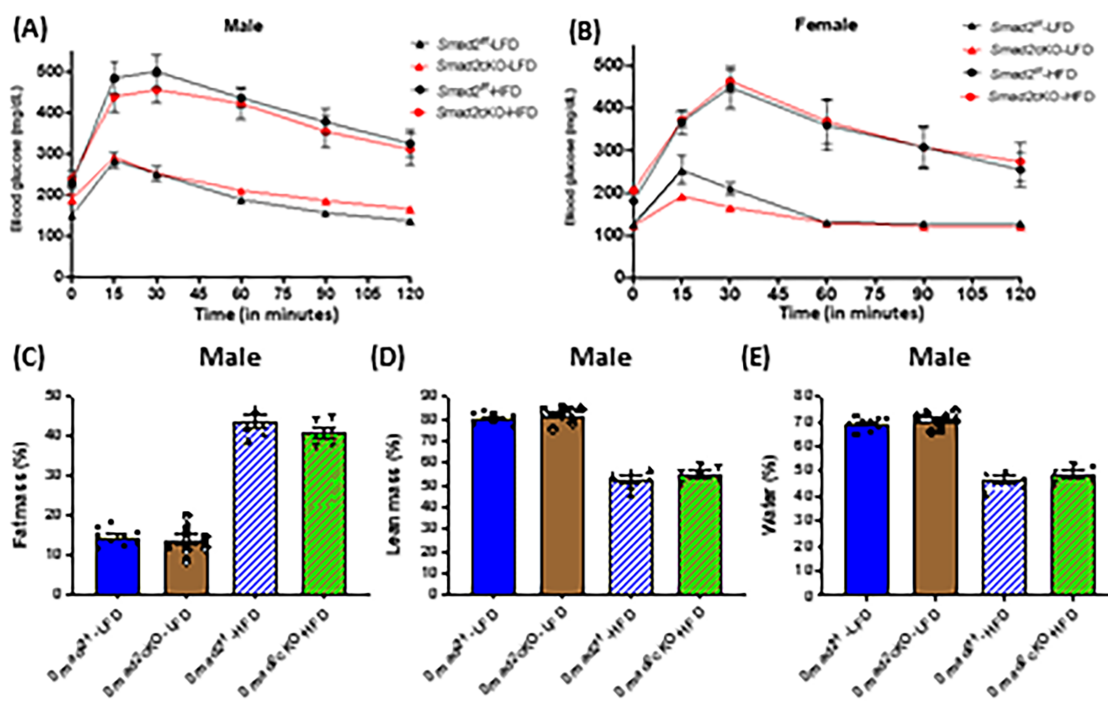

(D) Male

(E)
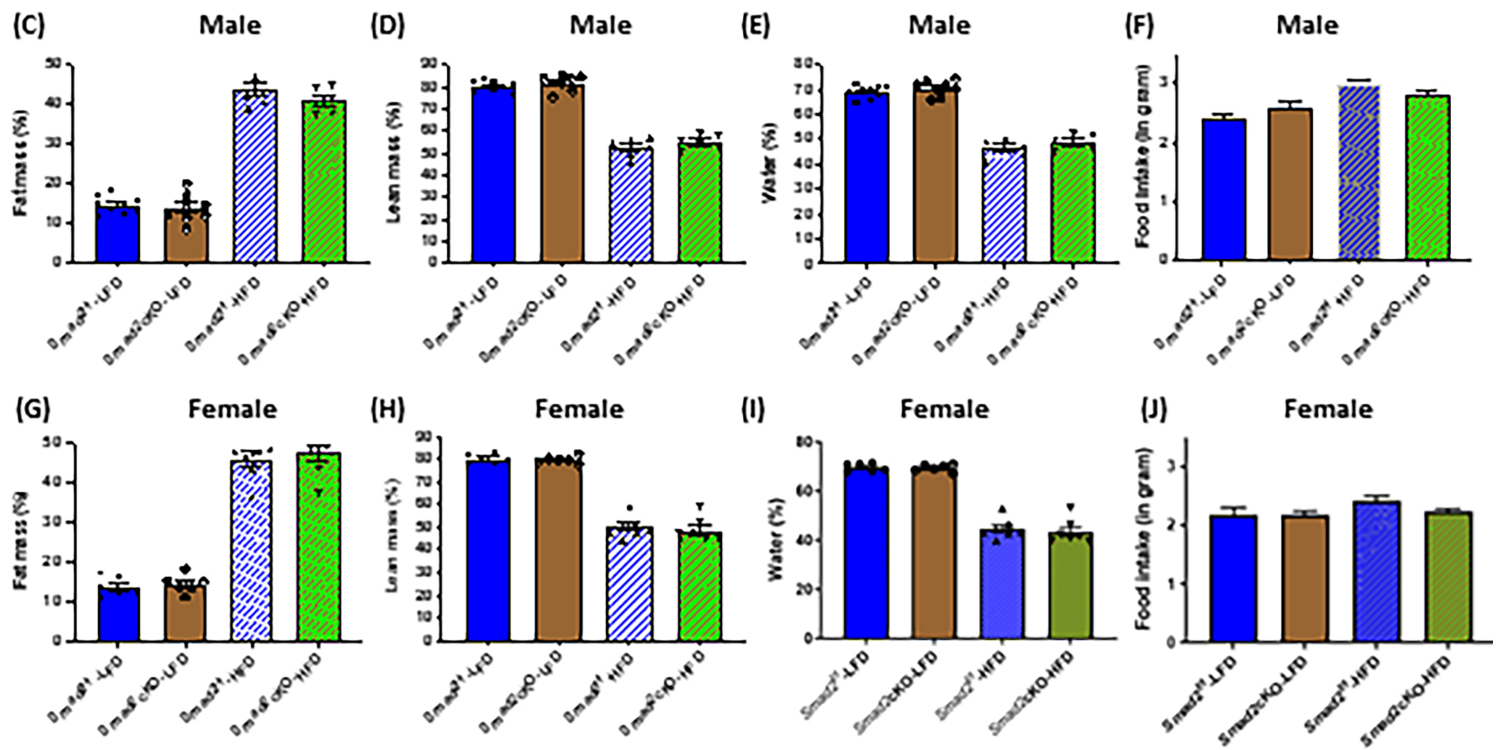

Figure 4.10: Smad2cKO Mice Show Similar Blood Glucose Levels and no Effect on Fat Mass. (A-B) GTT of Smad2cKO male and female mice. Echo MRI scan indicating no change in (C) fat mass, (D) lean mass, $(\mathrm{E})$ water, $(\mathrm{F})$ daily food intake in male and $(\mathrm{G})$ fat mass, $(\mathrm{H})$ lean mass, (I) water, (J) daily food intake in female. 
et al., 2011; Yadav, Quijano, et al., 2011). To determine if the differences in body weight between Smad3cKO and control mice were due to the differences in adipose tissue mass, we measured fat and lean mass by EchoMRI. Smad3cKO male mice exhibited significantly lower fat mass relative to controls on both LFD and HFD with slight increase in lean mass and water composition (Figure $4.11 \mathrm{~A}-\mathrm{C}$ ). In contrast, no differences were observed in fat mass, lean mass, water composition or water and food intake when comparing Smad3cKO and control female mice (Figure 4.12 A-D).

The differences in total body fat mass were also observed in the subcutaneous fat depots. Smad3cKO male mice showed significantly lower subcutaneous fat mass under LFD and HFD conditions. Visceral fat mass was lower in Smad3cKO mice on LFD, while Smad3cKO mice on HFD showed no difference in their visceral fat mass (Figure 4.11 DE). Smad3cKO mice showed a modest increase in HFD food intake that did not reach significance (Figure 4.11 F). The differences in subcutaneous fat mass were also reflected by adipocyte size. Subcutaneous WAT of Smad3cKO mice contained smaller, multiloculated adipocytes, irrespective of diet. In contrast, visceral WAT did not show clear differences in adipocyte size under either diet condition (Figure $4.11 \mathrm{G}$ ). Overall, these data are consistent with the lower body weight of Smad3cKO on HFD being attributable at least in part to the reduction in subcutaneous WAT mass.

Because of the depot-selective effects of Smad3 deficiency we further examined the mRNA levels of adipogenic markers in subcutaneous WAT from Smad3cKO mice. Under LFD conditions, all adipogenic markers, Сebp $\alpha, C e b p \beta, C e b p \delta$, and $P p a r \gamma$, trended lower than controls but with HFD these markers all increased relative to controls and relative to levels under LFD conditions, suggesting a possible higher sensitivity of Smad3cKO mice to HFD-related adipogenic effects (Figure 4.13 A-B). We further examined the mRNA level of genes involved in lipogenesis and lipolysis on both diets in subcutaneous WAT from Smad3cKO mice and control mice. Subtle differences were observed for several genes involved in lipogenesis and lipolysis on LFD and HFD. On HFD diet conditions, Acaca, Scd1, Hsl and Perilipin were slightly higher and lower expression of Fasn, Srebp1, Cd36 and Atgl in Smad3cKO mice, none of these markers reached significance. (Figure 4.13 C-D).

\subsection{Discussion}

We have demonstrated that SMAD2 and SMAD3 are not functionally interchangeable within the context of adipose tissue development, and SMAD3 likely plays the predominant role in subcutaneous white adipose tissue development and maintenance. Through their interactions with the 'common SMAD' (SMAD4), SMAD2 and SMAD3 are the main downstream transcriptional mediators of the activin signaling pathway (Zaragosi et al., 2010; Zamani and Chester W. Brown, 2011; M. J. Lee, 2018). Each protein, however, has acquired non-redundant functions. Smad2 global knockout mice die in utero because of gastrulation defects, while Smad3 global knockout mice are viable, demonstrating, as in other contexts, that the SMAD2 and SMAD3 proteins are not completely functionally interchangeable 

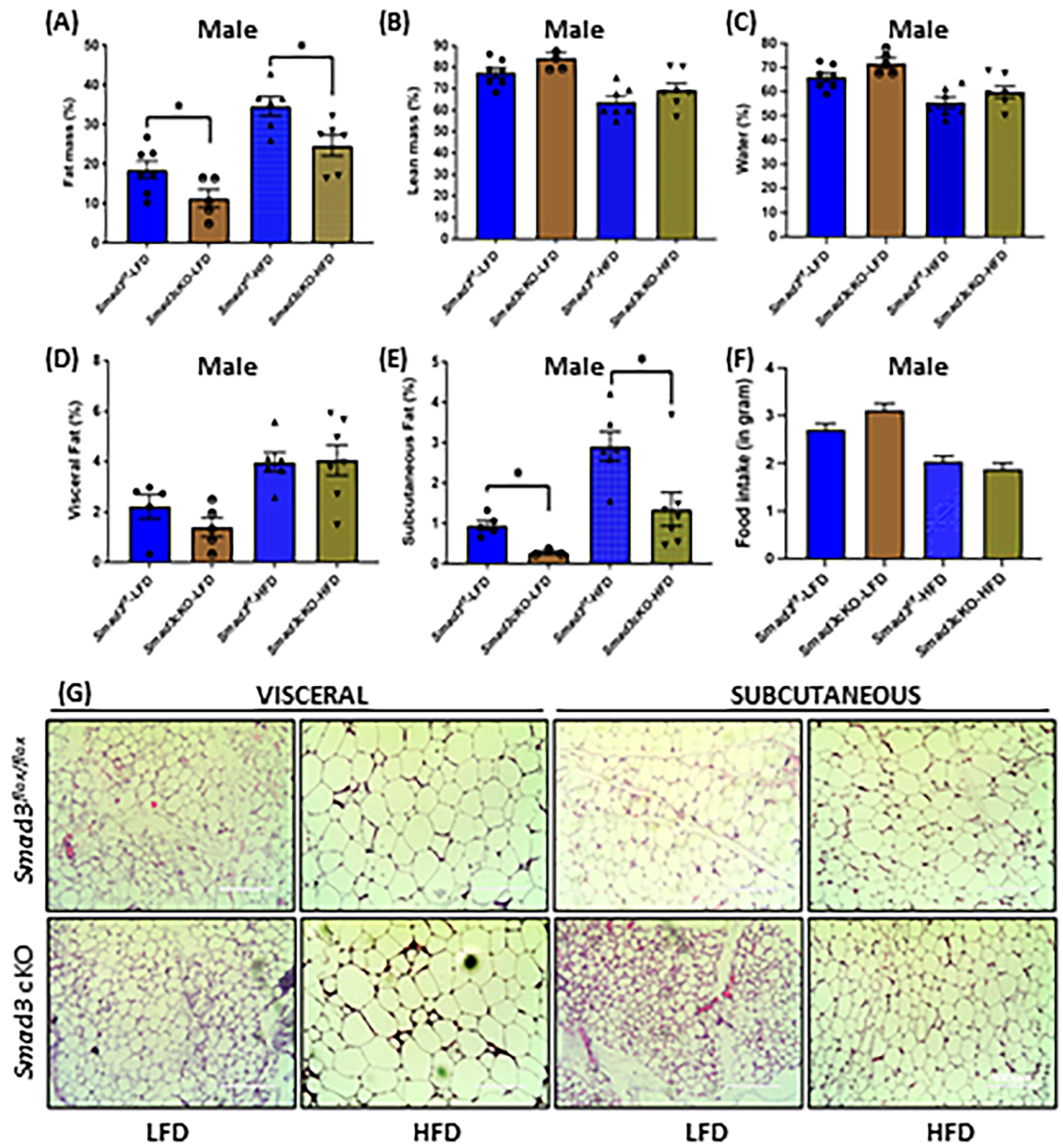

Figure 4.11: Adipocyte-Selective Deletion of Smad3 in Males Diminishes Subcutaneous WAT Irrespective of Diet. (A) Echo-MRI showing lower percent fat mass in Smad3cKO male mice than control mice fed LFD or HFD for 21 weeks. (B) No significant change in lean mass or (C) \% water composition. (D) Modest reduction in visceral fat mass on LFD. (E) Smad3cKO male mice displayed significantly lower subcutaneous fat mass on both LFD and HFD-fed (F) Daily food intake at 16 weeks. Smad3cKO male mice on LFD had slight increase in food intake while on HFD both groups had similar caloric intake ( $\mathrm{p}=0.054)$. $(\mathrm{G}) \mathrm{H} \& \mathrm{E}$ staining of visceral and subcutaneous adipose tissues at 23 weeks, showing smaller adipocytes in subcutaneous adipose on LFD and HFD $(n=4)$ while similar adipocyte sizes in visceral depots of Smad3cKO mice compared to control. Scale bar 400uM. 


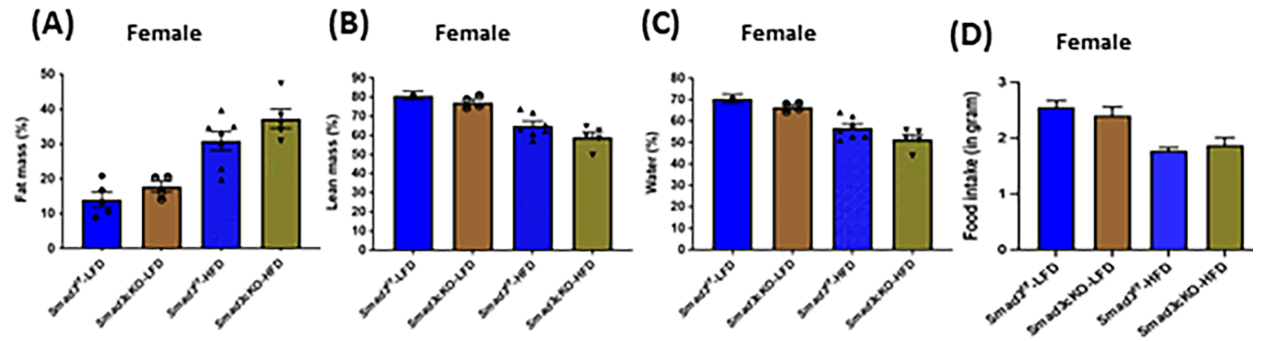

Figure 4.12: Smad3cKO Female Show No Effect on Fat Mass. Echo-MRI scan performed at 21week LFD and HFD fed mice indicating (A) no change in fat mass, (B) lean mass, (C) water composition and (D) daily food intake measured manually.

(A)
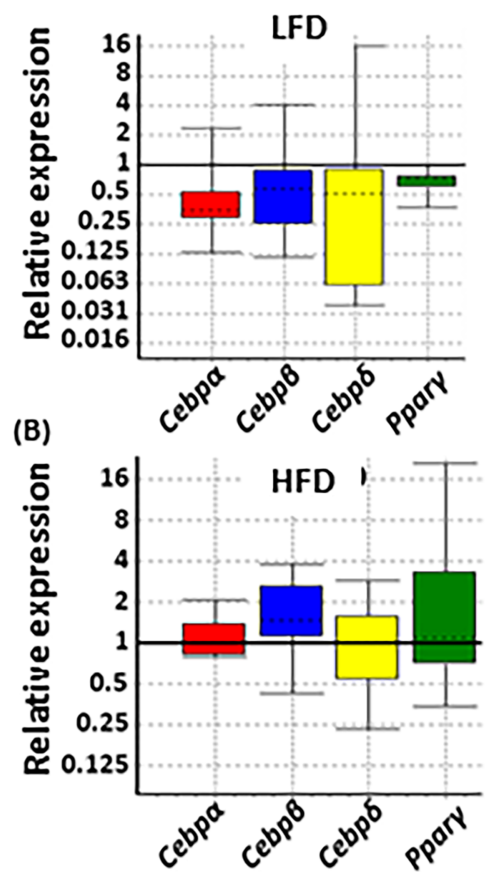

(C)

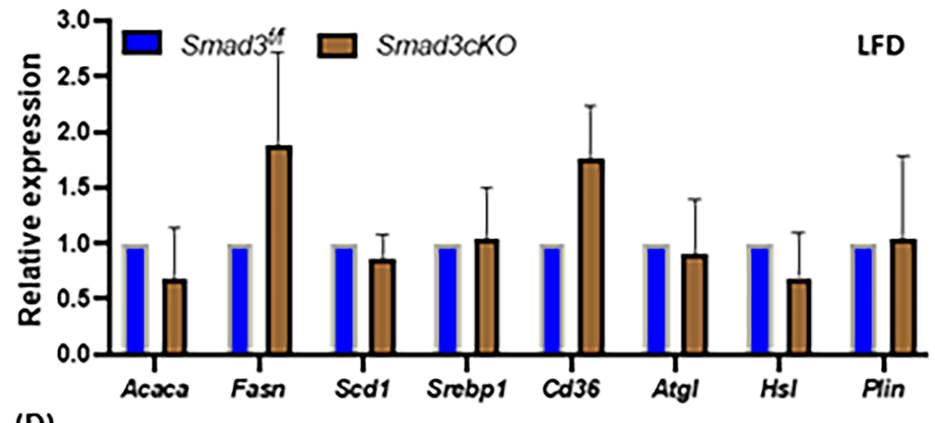

(D)

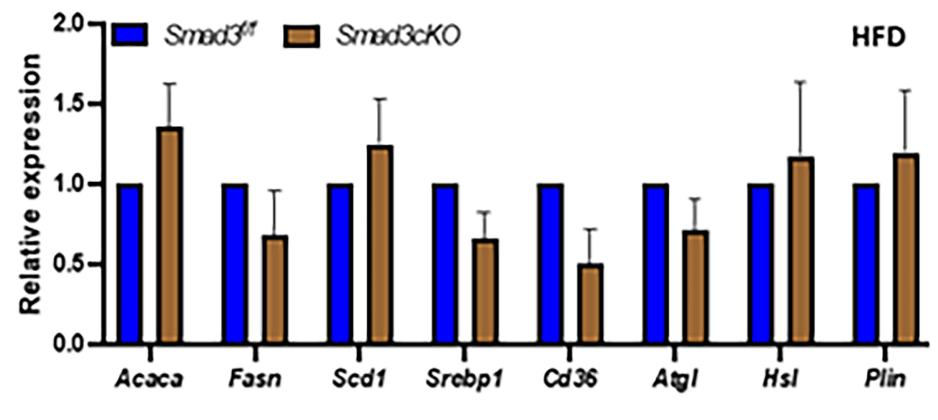

Figure 4.13: mRNA Levels of Adipogenic Markers in Subcutaneous WAT. (A-B) RT-PCR showing mRNA levels of adipogenic markers (C-D) mRNA levels of lipogenic and lipolytic markers of control and Smad3cKO mice $(n=4-5)$ on LFD and HFD fed for 23 weeks relative to the control value of one. Student's T-test was applied with $\mathrm{p}$ value $\leq 0.05$ considered as statistically significant for each experiment. 
(Nomura and E. Li, 1998; Waldrip et al., 1998; Weinstein et al., 1998; Y. Zhu et al., 1998; Datto et al., 1999; Heyer et al., 1999; C. K. Tan, Leuenberger, et al., 2011; Tsurutani et al., 2011; Yadav, Quijano, et al., 2011; Yadav and Rane, 2012; Yadav, Devalaraja, et al., 2017; J. H. Lee et al., 2020).

Previous studies have shown that Smad3 is required for the normal development of adipose tissue and normal somatic growth, as Smad3 global $\mathrm{KO}$ mice are small, have reduced adiposity and show protection from diet-induced obesity (Nomura and E. Li, 1998; Y. Zhu et al., 1998; Datto et al., 1999; C. K. Tan, Leuenberger, et al., 2011; Tsurutani et al., 2011; Yadav, Quijano, et al., 2011). However, this could be a secondary consequence of physiological effects from other tissues such as skeletal muscle, liver or pancreas (Y. Zhu et al., 1998; Datto et al., 1999; Yang et al., 1999; C. K. Tan, Leuenberger, et al., 2011; Tsurutani et al., 2011; Yadav, Quijano, et al., 2011; Yadav and Rane, 2012; Yadav, Devalaraja, et al., 2017; M. J. Lee, 2018; J. H. Lee et al., 2020). Therefore, we examined the tissue-selective effects of Smad2 and Smad3 on adiposity and diet-induced obesity.

Activin A promotes proliferation and inhibits differentiation via SMAD2 phosphorylation in 3T3-L1 preadipocytes and human adipocyte progenitors (Hirai et al., 2005; Zaragosi et al., 2010). To examine whether activins signal through both SMAD2 and SMAD3 phosphorylation during adipogenesis, we differentiated MEFs derived from activin-deficient mice (activin DKO) and surprisingly found significantly reduced levels of pSMAD2 but not pSMAD3. The reason that pSMAD3 levels remained unchanged in the absence of activins is unclear but could be due to maintenance of expression through the effects of other TGF- $\beta$ family ligands or due to a yet to be defined adipose-selective role for pSMAD2 that does not require pSMAD3 (Bonomi et al., 2012).

Signal transduction by several TGF- $\beta$ family members regulates adipogenesis in vivo and in vitro, including activins/SMADs (Choy, Skillington, and Derynck, 2000; Hirai et al., 2005; L. Li et al., 2009; Zaragosi et al., 2010; Zamani and Chester W. Brown, 2011; M. J. Lee, 2018; Aykul et al., 2020). However, depot-selective effects of activin on adipogenesis in preadipocytes have not been studied previously. Therefore, we differentiated preadipocytes isolated from different depots in the presence of exogenous activins. Herein, we examined the effect of activin/SMAD signaling on preadipocyte differentiation in visceral and subcutaneous WAT, revealing that subcutaneous and visceral preadipocytes respond differently and display different properties during adipogenesis with much higher differentiation efficiency in subcutaneous relative to visceral preadipocytes (Figures 4.4-4.5). There was higher lipid content in cells from the subcutaneous depot, while visceral preadipocytes produced fewer differentiation foci. Collectively, we show that activin A negatively impacts differentiation, whereas blocking activin signaling with SB431542 augments the differentiation of subcutaneous and visceral preadipocytes as shown by others in 3T3-L1 cells and human primary subcutaneous preadipocytes (Zaragosi et al., 2010; Merrick et al., 2019; Aykul et al., 2020). Since SB431542 blockade is not specific to activin signaling, inhibiting three of the type I TGF- $\beta$ family receptors, ALK4, ALK5 and ALK7, we cannot exclude 
the possibility that other ligands such as TGF- $\beta$ might also be impacted, although ALK4 and ALK7 are the type I receptors for activins, and ALK7 is the predominant type I activin receptor in adipocytes (Carlsson et al., 2009).

SMAD2 showed sustained expression in vivo and during adipocyte differentiation in vitro, while SMAD3 expression declined as adipocyte differentiation progressed in vitro and as adipose tissue growth progressed in both visceral and subcutaneous fat. This difference in temporal expression might suggest that SMAD3 plays a more critical role during early stages of preadipocyte specification and differentiation, whereas SMAD2 may play an overlapping or distinct role(s) at early stages and also important roles at later stages in mature adipocyte function and maintenance. It is clear that activin A treatment in primary subcutaneous preadipocytes diminishes differentiation. This is not as clear in visceral preadipocytes, but it is more challenging to interpret in the context of a cell population that already differentiates poorly at baseline. Similarly, the SMAD3 inhibitor showed a robust inhibitory effect in subcutaneous preadipocytes at the cellular and molecular levels, but not as clearly in visceral, although the relatively poor differentiation of visceral cells again makes interpretation challenging. Conversely, treatment with SB431542 greatly enhanced the differentiation of visceral cells at the cellular and molecular level, with a less robust effect in subcutaneous cells, which already achieve $90 \%$ differentiation efficiency at baseline. Thus, activin A and SMAD3 inhibition have similar effects in vitro, diminishing adipocyte differentiation, whereas SB431542, an inhibitor of 3 different type I receptors, enhances adipocyte differentiation. The similar effects of activin A and a SMAD3 inhibitor are counterintuitive, since SMAD3 is a downstream mediator of activin signaling. However, SMAD2 can also function in this capacity, so the result does not support a simple model of SMAD3-selective activin signaling. Unfortunately, to our knowledge, a specific SMAD2 inhibitor is not commercially available and additional experiments will be required to reconcile these observations. Irrespective, evidence for depot-selective effects for SMAD3 is most strongly provided by the in vivo experiments, showing disproportionate reduction of fat mass and cell size in subcutaneous WAT. Differences between the effects of SMAD2 and SMAD3 are best illustrated by no significant impact on weight gain in the Smad2cKO mice.

Using Smad2cKO and Smad3cKO, we have shown that adipose tissue-selective reduction of Smad2 or Smad3 using Adiponectin-Cre had different effects on diet-induced weight gain. Body weight and fat mass of Smad2cKO male and female mice were not different from control littermates. This suggests that Smad2 plays little to no role in maintaining adipose tissue during mid to late adipogenesis in vivo. In contrast, Smad3cKO mice demonstrated a sex-dependent effect on weight gain. Smad3cKO male mice gained less weight relative to control littermates on HFD, while females were similar to control mice, indicating a sex-influenced role for $S m a d 3$ on adiposity. The phenotypic differences between the adiposeselective Smad3cKO and the Smad3 global KO model reported by others (including no report of sexual dimorphism) may be due to secondary physiological effects that occur in the global knockout, the more restricted perturbation of expression in the Smad3cKO, or differences in the timing of SMAD3 reduction in the two models. 


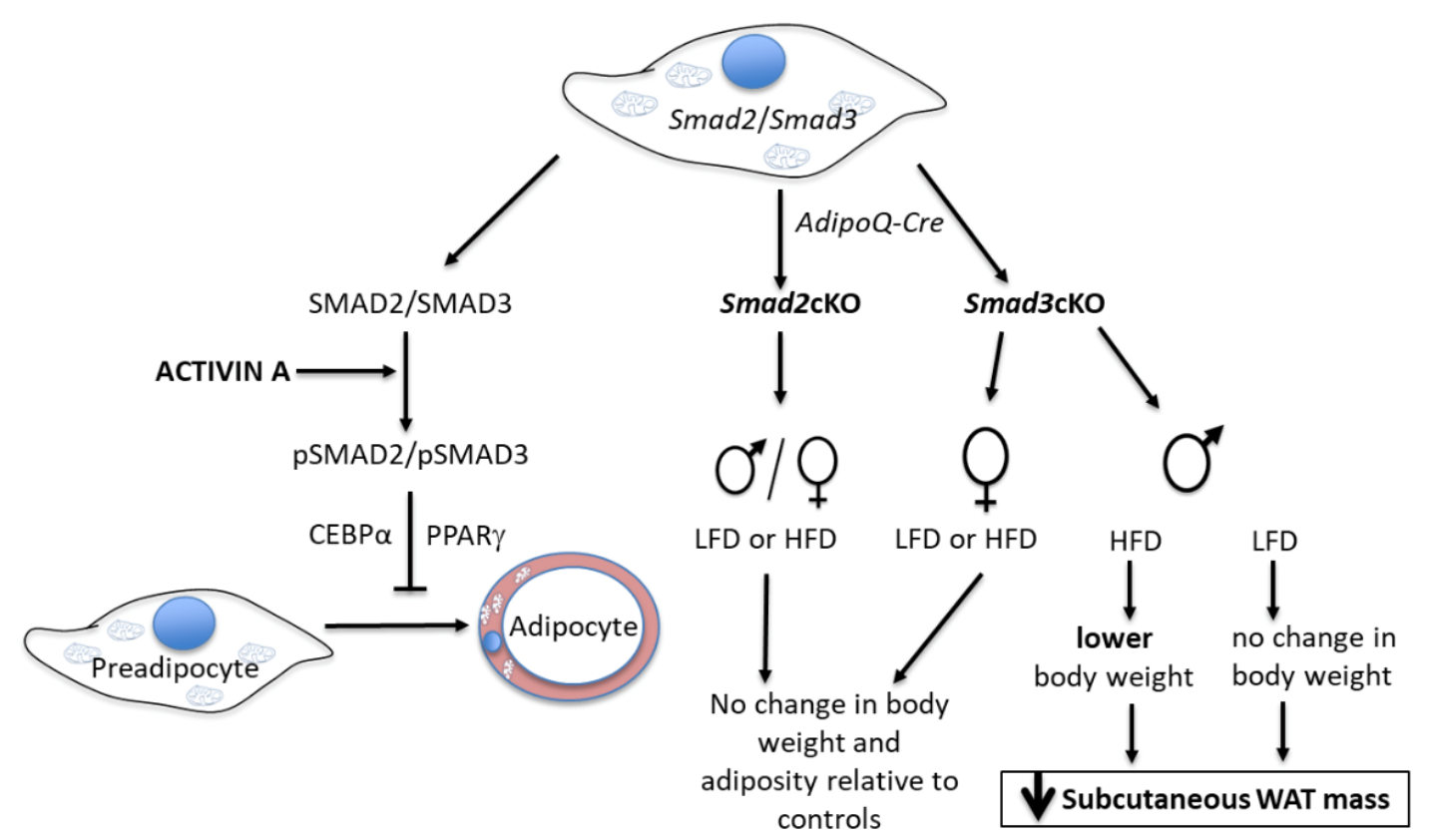

Figure 4.14: Working Model Showing Effects of SMAD2 and SMAD3 on White Adipose Tissue. SMAD2 and SMAD3 expressed in preadipocytes are phosphorylated by Activin A and thereby inhibit adipocyte differentiation by suppressing Ppar $\gamma$ and Cebpa. Adipocyte-selective, Adipoq-Cre-mediated deletion of Smad2 does not impact white adipose tissue (WAT) development or diet-induced obesity, while adipocyte-selective deletion of Smad3 diminishes the subcutaneous (but not visceral) white adipose tissue mass in male.

Smad3cKO male mice exhibited decreased adiposity on HFD with less subcutaneous fat and no difference in visceral fat relative to controls. Lean mass increased slightly on both diets and water composition did not differ, nor did food intake. These findings are consistent with the lower body weight of Smad3cKO male mice on HFD being due at least in part to the reduced accumulation of subcutaneous WAT mass. The reason for the predominant effect in subcutaneous fat is unclear but may be due to intrinsic differences between the depots in their requirements for SMAD3-mediated signaling or differences in Adiponectin-promoter-driven Cre expression in visceral and subcutaneous fat depots. This notion is consistent with an earlier report that the Adiponectin promoter is active during fetal subcutaneous adipose tissue development but postnatally in the visceral depot (M. Shao et al., 2017). In summary, our findings indicate that adipose-selective Smad3 but not Smad2 deficiency results in significantly lower subcutaneous WAT mass, suggesting an important selective role of SMAD3 in subcutaneous WAT development in vivo (Figure 4.14). 
One potential limitation of this study is our decision to use the Adiponectin-Cre mouse. For the purpose of achieving adipose-selective gene inactivation, and for studying events that occur in the middle to late stages of adipogenesis and in mature adipocytes, it has been informative. However, it is plausible that SMAD-mediated events occurring at earlier stages of adipogenesis have not been substantially impacted in our model. Thus, to fully understand the role of SMAD-mediated signaling and associated molecular mechanisms that occur during earlier stages, additional studies are needed using alternative strategies. This will allow us to investigate specific deletion of SMAD2 and SMAD3 individually or in combination at specific time points to better understand the complex interrelationship between SMAD2, SMAD3, adipocyte development and function. 


\section{Chapter 5}

\section{Combinatorial Role of SMAD2/3 on Adiposity}

\subsection{Introduction}

SMAD2 and SMAD3 are almost structurally identical downstream transduction signals of TGF- $\beta$ /activin signaling. As discussed in chapter 2 and 3, depending on the cellular context, these two transcription factors may have similar, unique, or overlapping roles depending on which SMAD complex is present in each tissue (Y. Shi and J. Massagué, 2003; Q. Li, Pangas, et al., 2008; Kriseman et al., 2019; Kroon et al., 2017; S. Huang, Chen, Su, et al., 2019; S. Huang, Chen, Humeres, et al., 2020). In the previous chapter, we studied the effect of adipose selective single knockout of Smad2 and Smad3 on adipose tissue function and observed that Smad3 knockout partially protected the male mice from diet-induced obesity (DIO) while female cKO mice showed no effect on diet-induced obesity. Smad2 showed little to no role on adipose tissue during mid to late stages of adipogenesis. This suggested that SMAD2 and SMAD3 have at least some distinct roles in adipose tissue. However, we do not fully understand the combinatorial requirement of SMAD2/3 in adipose tissue development and function. Herein, we discuss the impact of combined adipose selective deletion of Smad2/3 during mid to late adipogenesis.

\subsection{Results}

\subsubsection{Adipose-Selective Deletion of Smad2/3 Protects Mice from Diet-Induced Obesity in a Sex-Specific Manner}

To explore the adipose-selective combined effects of SMAD2/3, we generated Smad2/3 double conditional knockout mice (hereafter, Smad2/3cKO) using Adiponectin-Cre and deletion was confirmed by western blot (Figure 5.1). Adiponectin-Cre-mediated reduction of SMAD2 and SMAD3 was confirmed by RT-PCR and western blot. First, we investigated whether the adipose tissue-selective reduction of $S m a d 2 / 3$ results in any survival or growth defect. Smad2/3cKO mice did not show any obvious growth defects under regular diet conditions (Envigo Lab Diet 7912), and the mice were healthy and fertile. Next, we examined the effects of Smad2/3 under HFD and LFD conditions. Smad2/3flox / flox (control) and Smad2/3cKO mice 


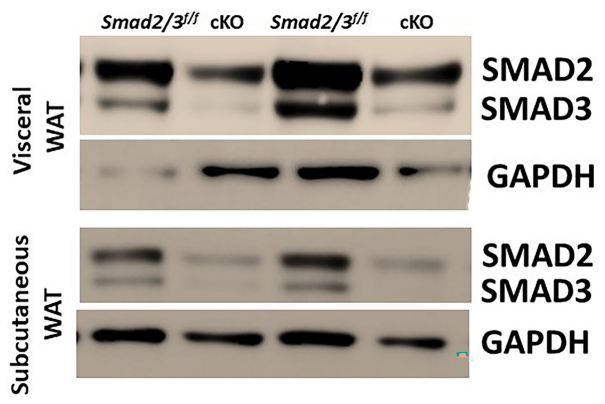

Figure 5.1: Generation of Smad2/3cKO Mice Using Adiponectin-Cre. Western blot showing efficiency of Adiponectin-Cre for Smad2/3 in both visceral and subcutaneous WAT.
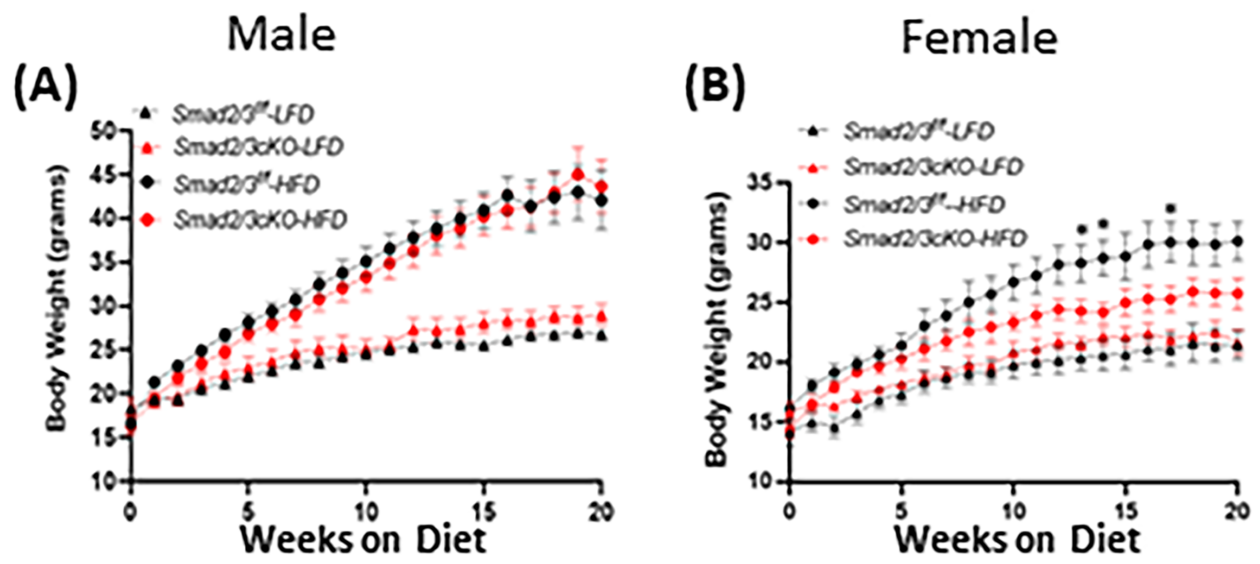

Figure 5.2: Lower Body Weight in Smad2/3cKO Mice in Females. Growth curves showing (A) no detectable differences in the body weights of Smad2/3cKO male mice relative to control mice fed HFD and LFD, $n=6-8$, (B) lower body weight in Smad2/3cKO females on HFD compared to controls, $\mathrm{n}=6-8$. 
were fed LFD or HFD for 22 weeks starting from the age of 5 weeks. As predicted, these mice normally remained lean on LFD diet, while HFD resulted in diet-induced obesity (DIO) over time. Surprisingly, in contrast to the earlier observed phenotype for Smad3cKO male mice, adipose-selective Smad2/3 deletion did not show any effect on body weight in males. In contrast to males, however Smad2/3cKO female mice on HFD displayed divergence in body weight starting at 6 weeks and continued with lower body weight gain relative to diet-matched controls with significant difference in body weight at weeks 13,14 and 17 and continued to show this divergence beyond 20 weeks (Figure 5.2 A-B). This demonstrates that $S m a d 2 / 3$ CKO female mice on HFD exhibit partial protection from DIO.

\subsubsection{Smad2/3-Deficient Female Mice Exhibit Differences in Fat Mass along with Weight Gain}

To determine if the differences in body weight between Smad2/3cKO and control female mice on HFD were due to the differences in adipose tissue mass, we measured fat and lean mass by EchoMRI. In the beginning when both diets were started, Smad2/3cKO female mice had same fat mass as control littermates. However as the mice continued with HFD, Smad2/3cKO female mice exhibited lower fat mass relative to controls on HFD with slight increase in lean mass and water composition at 16 weeks and 22 weeks on HFD compared to control littermates (Figure 5.3 A-I). In contrast, no differences were observed in fat mass, lean mass, and water composition when comparing Smad2/3cKO and control male mice in the beginning at 5 weeks or at 16 weeks till the time of tissue harvest at 22 weeks. (Figure $5.4 \mathrm{~A}-\mathrm{J})$.

Normally, DIO results in glucose intolerance. To determine whether this was the case in Smad2/3cKO female mice, we measured blood glucose levels at baseline and after glucose injection. Smad2/3cKO mice showed similar fasting blood glucose levels as controls at baseline and after glucose injection, lower blood glucose levels were consistantly observed in Smad2/3cKO female mice on HFD at initial 60 minutes and then merged at 90 minutes. No difference was detected in blood glucose levels under LFD conditions (Figure 5.5 A-C). Two-way (mixed model) ANOVA followed by post hoc test were used to evaluate statistical significance. To determine whether the improvement in blood glucose levels in Smad2/3cKO mice was due to improved insulin sensitivity, insulin tolerance testing (ITT) was performed in females. Smad2/3cKO mice did not show any differences in blood glucose levels relative to controls after insulin injection (Figure 5.5 D).

To determine the effect of Smad2/3 deletion on adipose tissue in females, visceral and subcutaneous WAT were also dissected from $S m a d 2 / 3 \mathrm{cKO}$ and control mice on both diets. Consistent with the reduction in fat mass in Smad2/3cKO female on HFD, Smad2/3cKO female mice showed lower visceral and subcutaneous fat mass under HFD conditions relative to LFD or HFD control littermates, however the decrease in fat mass from both the depots did not reach statistical significance (visceral, $\mathrm{p}=0.053$ ) (Figure $5.5 \mathrm{E}$ ). Taken together these results suggest that $S m a d 2 / 3 \mathrm{cKO}$ mice have reduced adiposity on HFD. The 
(A)

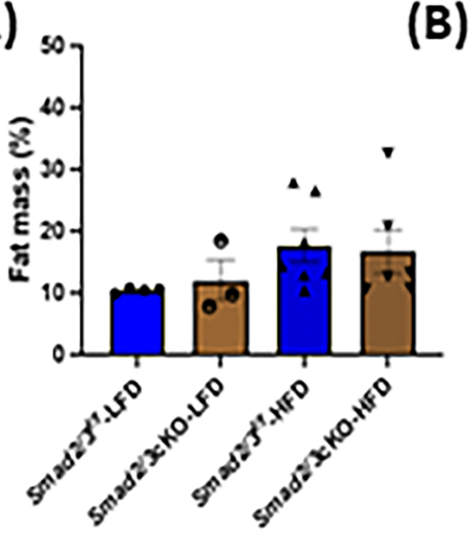

(D)

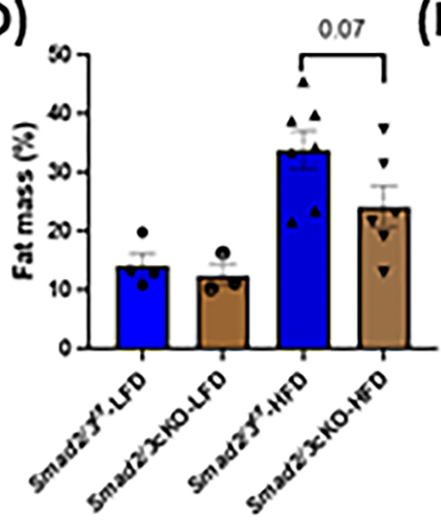

(G)

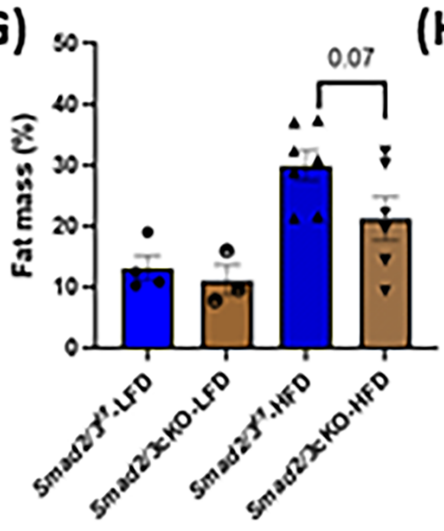

(B)

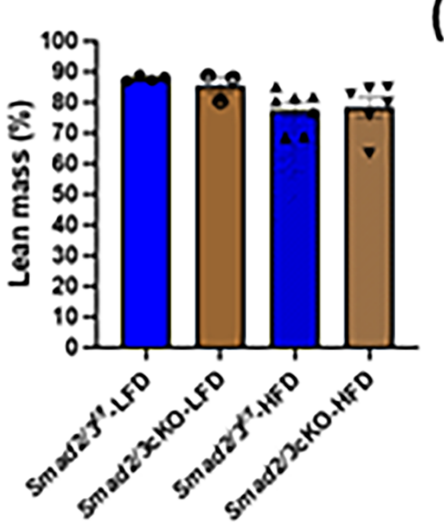

(E)

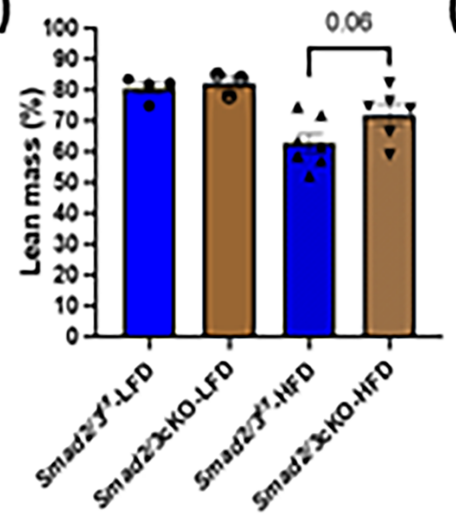

(H)

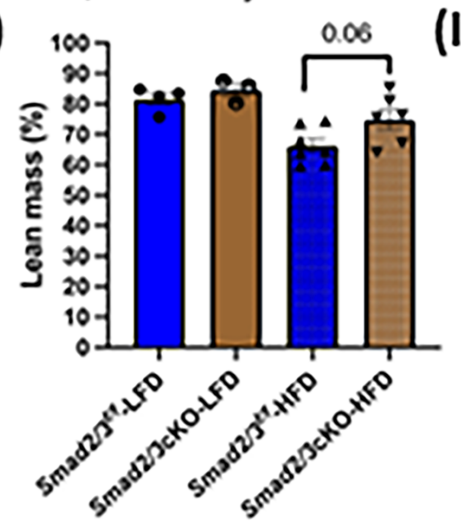

(C)

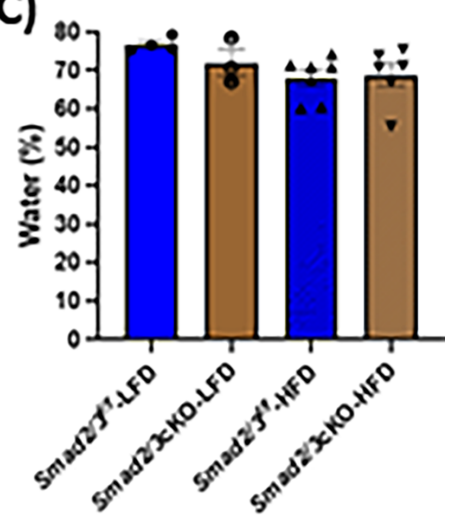

(F)

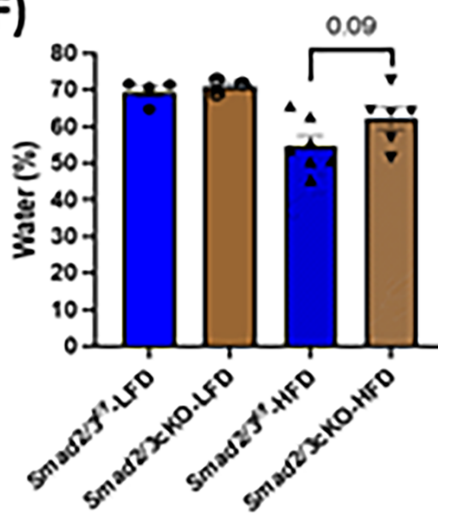

(I)

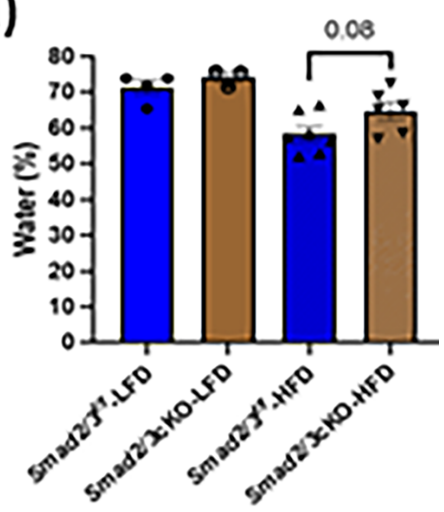

Figure 5.3: Lower Percent Fat Mass in Smad2/3cKO Mice in Females.

Echo-MRI showing (A-C) fat mass, lean mass, and water composition at 5 weeks, (D-F) 16 weeks and (G-I) 22 weeks of Smad2/3cKO female mice and control mice, $\mathrm{n}=6-8$. 
(A)

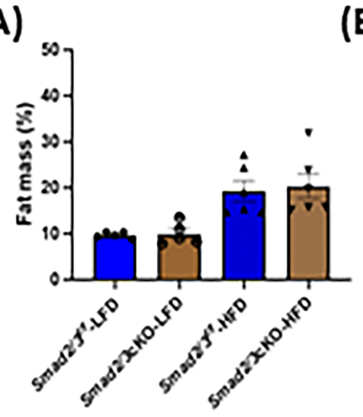

(D)

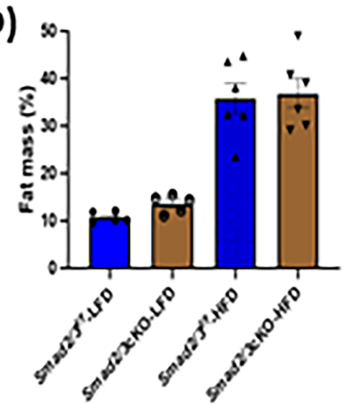

(B)

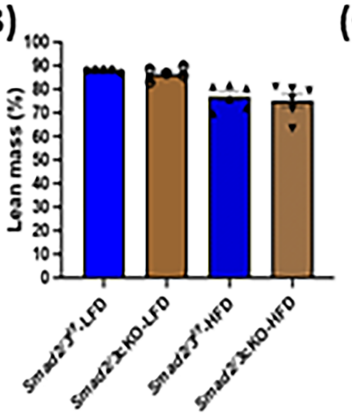

(E)

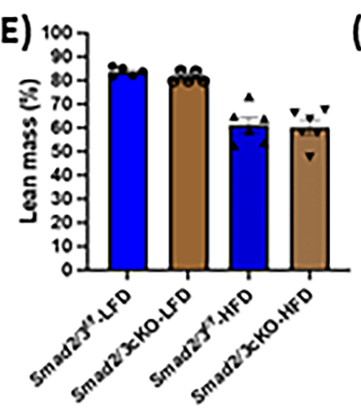

(C)

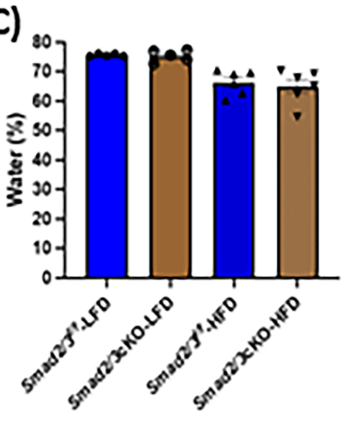

(F)

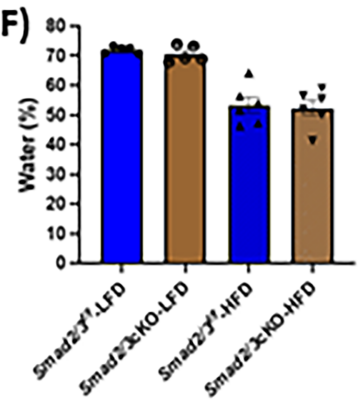

(G)

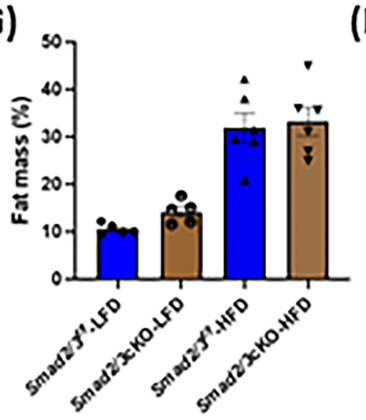

(H)

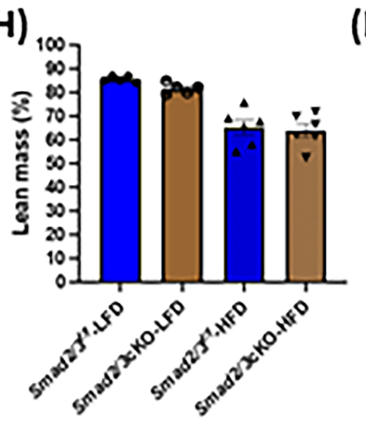

(I)

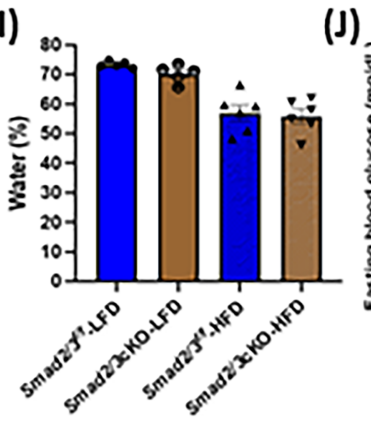

(J)

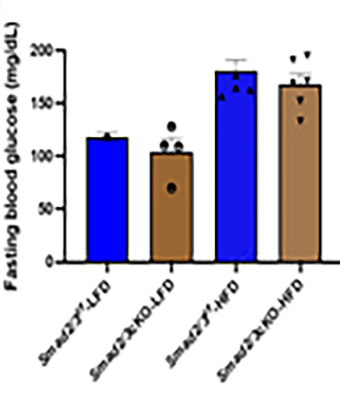

Figure 5.4: Adipocyte-Selective Deletion of Smad2/3 in Males Exhibits no Change in Fat Mass. Echo-MRI showing (A-C) fat mass, lean mass and water composition at 5 weeks, (D-F) 16 weeks and (G-I) 22 weeks of Smad2/3cKO male mice and control mice, $n=6-8$. (J) No detectable change in fasting blood glucose level in GTT. 


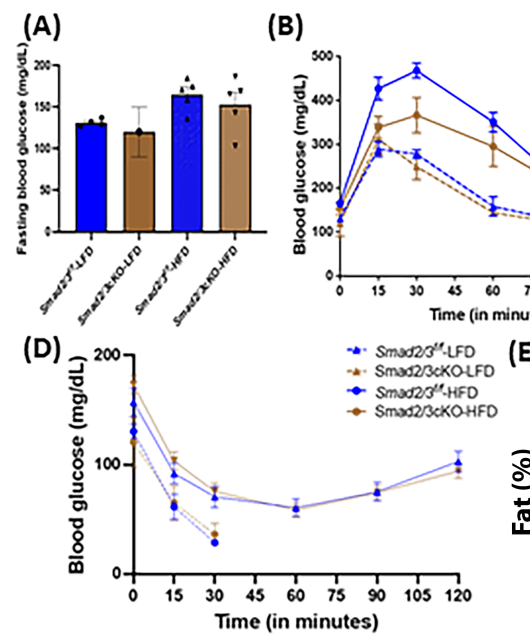

(c)

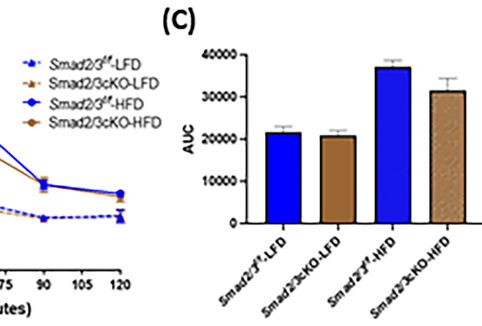

$(\mathbf{F})$
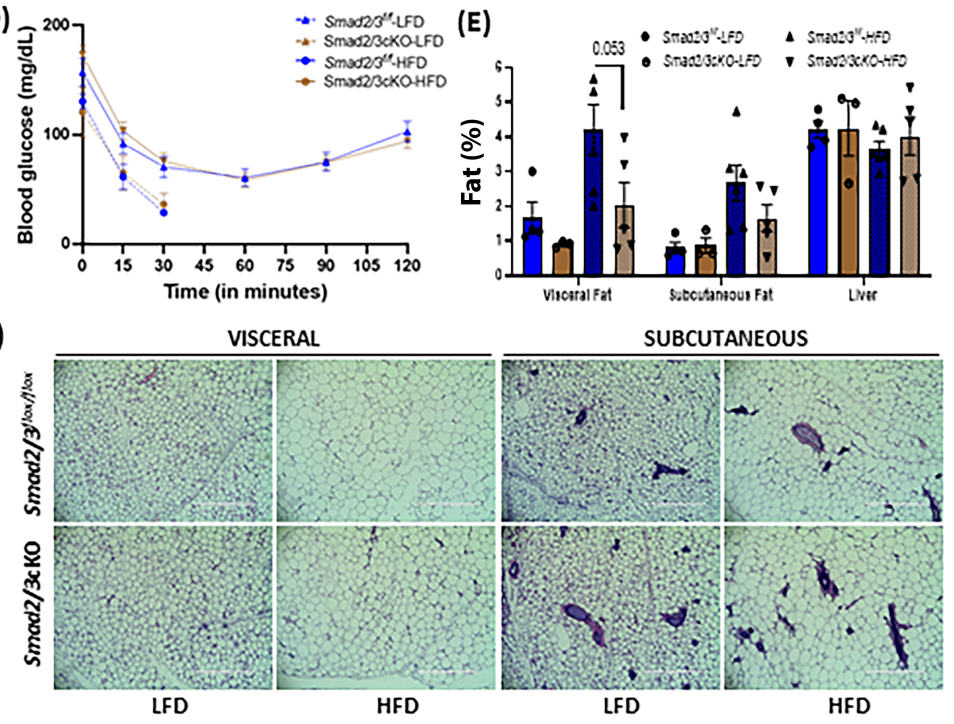

(G)

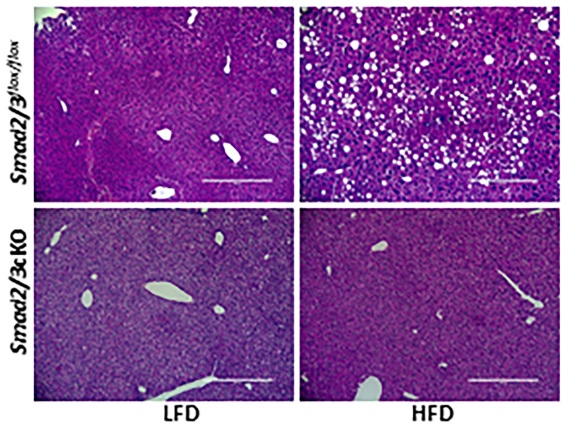

(H) Female-Food intake

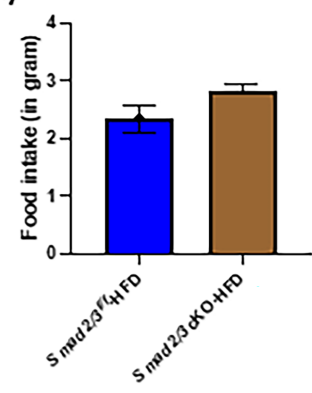

Figure 5.5: Diminished Visceral and Subcutaneous WAT in Smad2/3cKO Mice on HFD. Glucose tolerance test (GTT) showing (A-B) baseline blood glucose and after injection (C) area under the curve (AUC). (D) Insulin tolerance test (ITT). (E) Reduction in visceral (0.053) and subcutaneous fat mass on HFD but not significant. (F) $\mathrm{H}$ \& E staining of visceral and subcutaneous adipose tissues at 22 weeks of $S m a d 2 / 3 \mathrm{cKO}$ relative to control female mice on LFD and HFD ( $n=4)$. Scale bar 400uM. (G) H \& E staining of liver of Smad2/3cKO females. (H) Daily food intake on HFD. 
differences in visceral fat mass were also reflected by adipocyte size. Visceral WAT of Smad2/3cKO female mice on HFD contained relatively small multiloculated adipocytes. In contrast, subcutaneous WAT did not show clear differences in adipocyte size under either diet condition (Figure 5.5 F).

Often, DIO results in hepatic steatosis due to ectopic fat deposition in liver. Therefore, we analyzed the effect of Smad2/3 deletion on hepatic steatosis by H\& E staining. HFD, control mice showed prominent hepatic steatosis (white fat patches). Loss of SMAD2/3 in adipose tissue, however, resulted in protection from diet-induced hepatic steatosis (Figure 5.5 G). To determine if lower body weight in Smad2/3cKO mice is due to lower caloric intake, we measured food intake. Smad2/3cKO mice showed a modest increase in HFD food intake that did not reach significance (Figure $5.5 \mathrm{H}$ ). Overall, these data are consistent with the lower body weight of Smad2/3cKO female mice on HFD being attributable at least in part to the reduction in total WAT mass, including visceral and subcutaneous, and reduction of $S m a d 2 / 3$ in adipose results in indirect effects on hepatic lipid metabolism or storage.

\subsubsection{Smad2/3cKO Mice Show Increased Energy Expenditure}

Despite modest increase in food intake, Smad2/3cKO female mice on HFD showed lower body weight and reduced adiposity. This finding prompted us to investigate energy expenditure, substrate utilization and activity in Smad2/3cKO female mice. We performed 24 hour indirect calorimetry for seven days. All metabolic parameters were measured in 22-week-old mice under fed and fasted conditions. Data is presented as an average of all days (left panel) and average at every indicated time points per mouse (right panel), $\mathrm{n}=6$. Values normalized to body weight during both the light and dark cycle. Smad2/3cKO mice showed greater oxygen consumption $\left(\mathrm{VO}_{2}\right)$, carbon dioxide production $\left(\mathrm{VCO}_{2}\right)$, and total energy expenditure (TEE), indicative of higher metabolic rates (Figure $5.6\left(A-A^{\prime}\right)-\left(C-C^{\prime}\right)$ ). The differences in $\mathrm{VO}_{2}, \mathrm{VCO}_{2}$, and energy expenditure persisted in light and dark cycles while no effect of genotype was observed on physical activity (Figure 5.6 D-D')).

We further determined if there were any differences in substrate utilization. Smad2/3cKO mice showed higher respiratory exchange ratio (RER) (0.75) compared to controls (0.70) indicating that Smad2/3cKO mice use higher carbohydrate as an energy source than control mice (Figure 5.6 E-E'). Therefore, differences in energy substrate utilization may be contributing to the reduced adiposity in Smad2/3cKO mice. Considering the difference in body weight and fat mass, it is plausible that reduced adiposity in Smad2/3 conditional knockout mice is due to higher oxygen consumption and total energy expenditure.

Collectively, these results suggest that $S m a d 2 / 3$ deletion improves metabolic rate of female mice. We also looked at these metabolic parameters under fasting conditions, and found the same results. Smad2/3cKO mice showed greater $\mathrm{VO}_{2}, \mathrm{VCO}_{2}$ and total energy expenditure. However, all three parameters declined as these mice continued to fast. Activity level increased for first few hours then declined. No difference was observed in the 

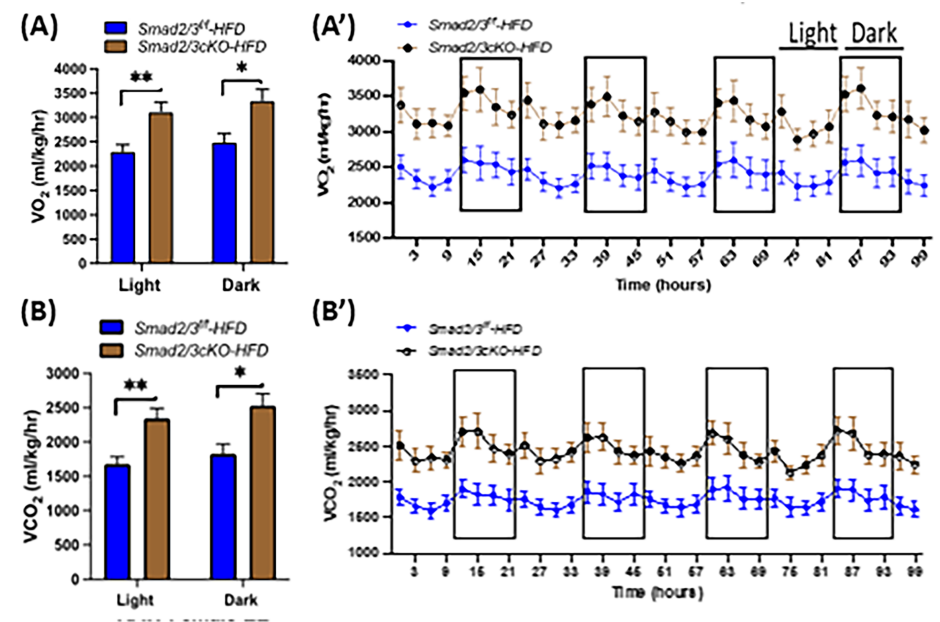

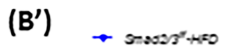
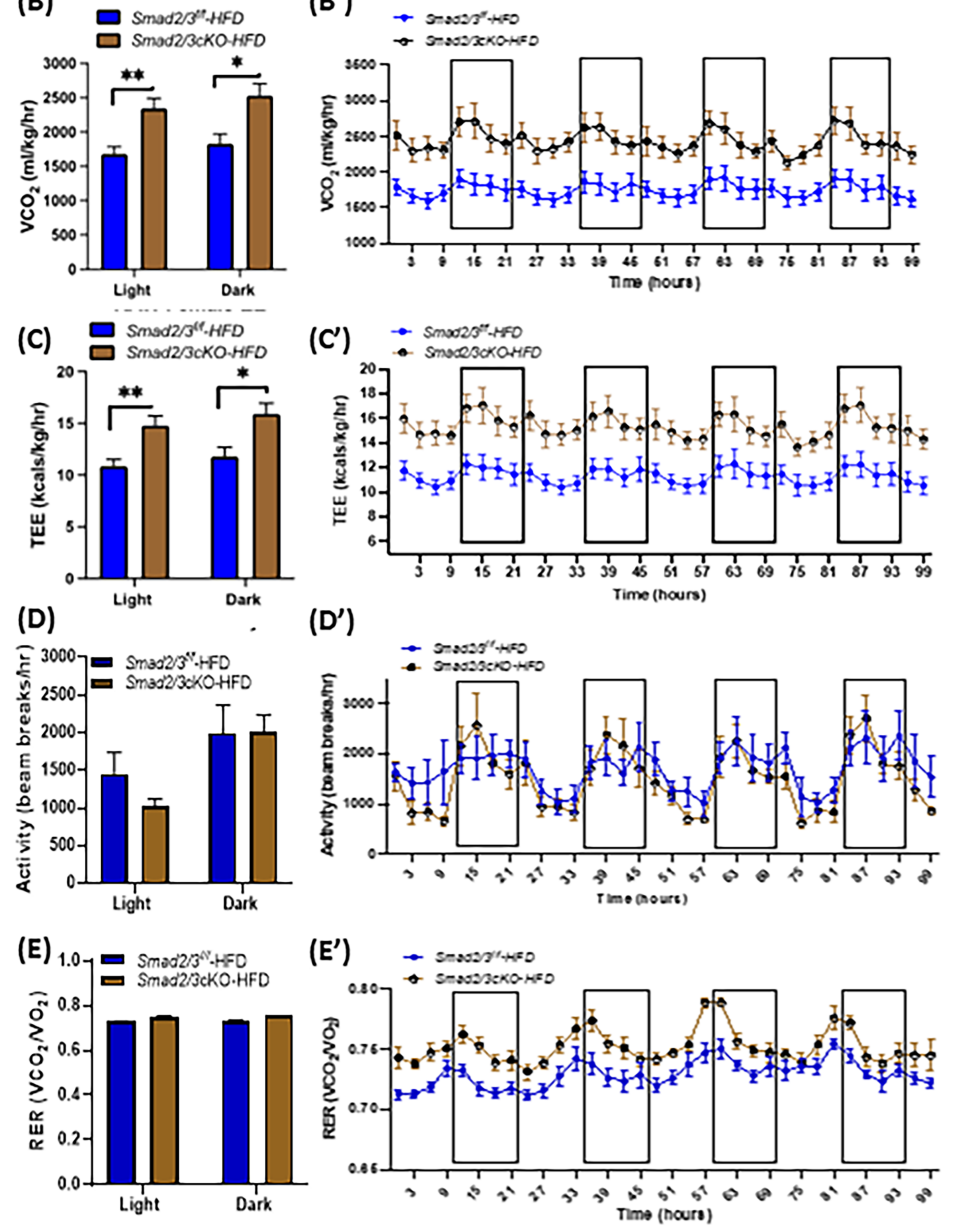

Figure 5.6: Indirect Calorimetry Showing Higher Metabolic Rate in Smad2/3cKO Mice on HFD. (A-A') $\mathrm{O}_{2}$ consumption rate $\left(\mathrm{VO}_{2}\right),\left(\mathrm{B}-\mathrm{B}^{\prime}\right)$ carbon dioxide production $\left(\mathrm{VCO}_{2}\right),\left(\mathrm{C}-\mathrm{C}^{\prime}\right)$ total energy expenditure (TEE), $\left(\mathrm{D}^{-} \mathrm{D}^{\prime}\right)$, total activity and $\left(\mathrm{E}-\mathrm{E}^{\prime}\right)$ respiratory exchange ratio (RER) of Smad2/3cKO and control female mice on HFD. Data are expressed as mean \pm standard error of the mean (SEM). $\mathrm{p}$ value $\leq 0.05$ considered as statistically significant, ${ }^{*} \mathrm{p}$ value $\leq 0.05,{ }^{* *} \mathrm{p}$ value $\leq 0.005$. 


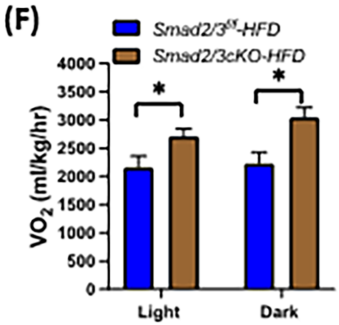

(H)

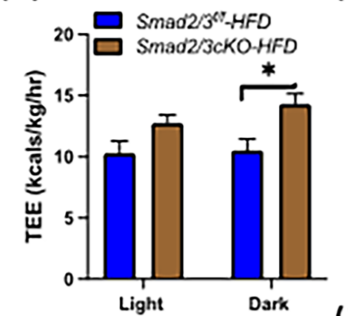

(J)

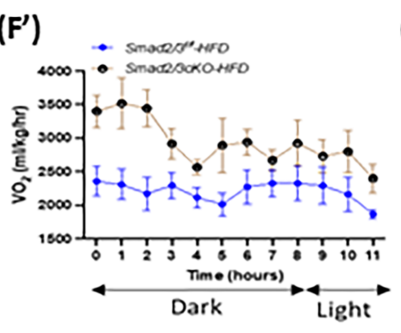

$\left(\mathrm{H}^{\prime}\right)$
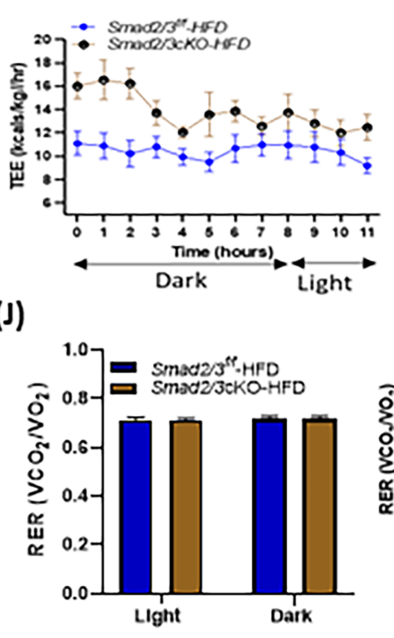

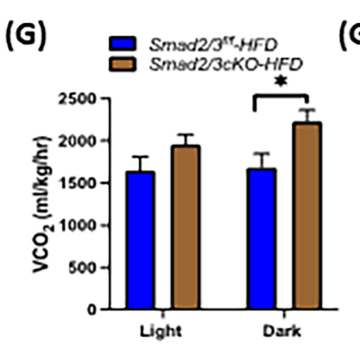

(G')

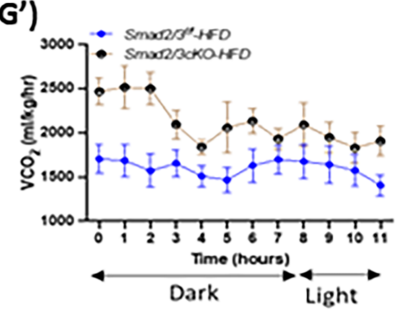

(I)

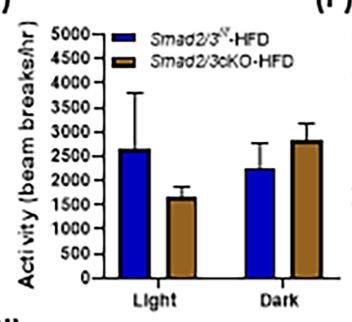

$\left(I^{\prime}\right)$

$\left(J^{\prime}\right)$

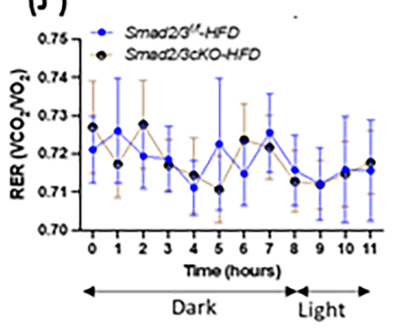

Figure 5.7: Indirect Calorimetry Showing Higher Metabolic Rate in Smad2/3cKO Mice on HFD in Fasting State. (F-F') $\mathrm{O}_{2}$ consumption rate $\left(\mathrm{VO}_{2}\right),\left(\mathrm{G}-\mathrm{G}^{\prime}\right)$ carbon dioxide production $\left(\mathrm{VCO}_{2}\right),\left(\mathrm{H}-\mathrm{H}^{\prime}\right)$ total energy expenditure (TEE), (I-I') total activity and $\left(\mathrm{J}^{\prime} \mathrm{J}^{\prime}\right)$ respiratory exchange ratio (RER) of Smad2/3cKO and control female mice on HFD in fasting conditions. Data is presented as an average of all days (left panel) and average at every indicated time points per mouse (right panel), $n=6$. Values normalized to body weight during both the light and dark cycle. Data are expressed as mean \pm standard error of the mean (SEM). Two-way mixed model ANOVA were used for multiple comparisons among genotypes overtime) and student's T-test was applied with $p$ value $\leq 0.05$ considered as statistically significant for each experiment. 

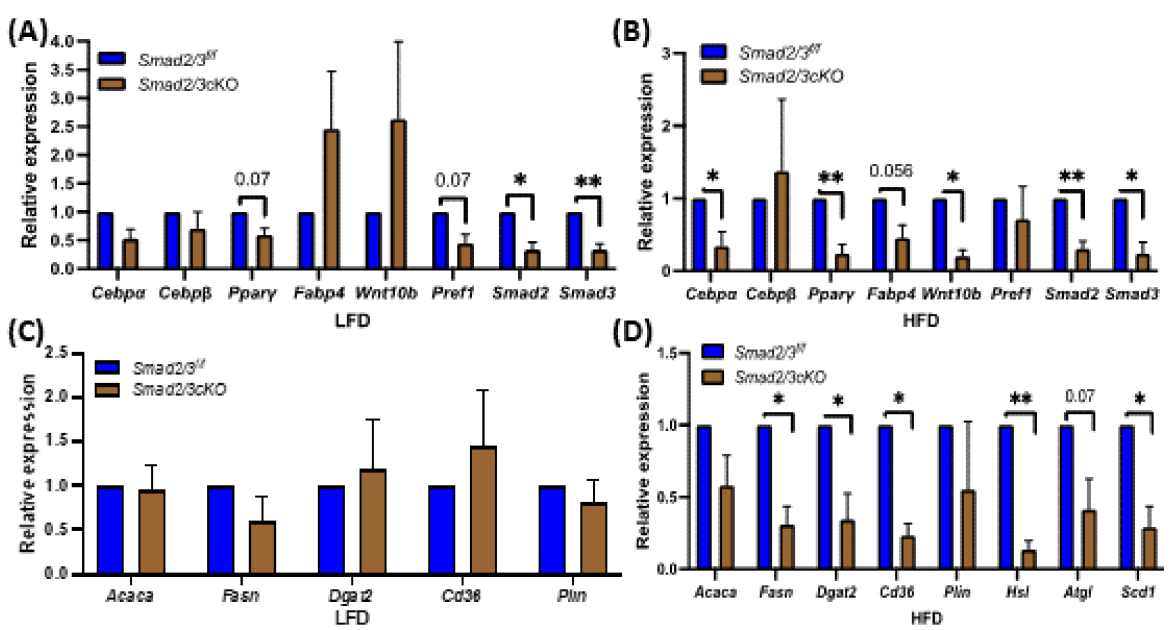

(D)

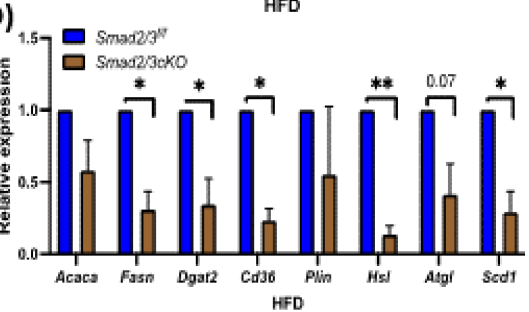

Figure 5.8: SMAD2/3 Regulate Key Genes Involved in Adipogenesis, Lipogenesis and Lipolysis. (A-B) mRNA levels of adipogenic markers

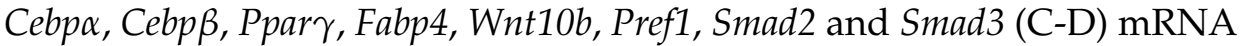
levels of lipogenic and lipolytic markers Acaca, Fasn, Dgat2, Cd36, Plin, Hsl, Atgl and Scd1 in subcutaneous WAT of control and Smad2/3cKO mice $(\mathrm{n}=4-5)$ on LFD and HFD fed for 22 weeks. Student's T-test was applied with $\mathrm{p}$ value $\leq 0.05$ considered as statistically significant. ${ }^{*} \mathrm{p}$ value $\leq 0.05,{ }^{* *} \mathrm{p}$ value $\leq 0.01$

respiratory exchange ratio (RER) in fasting condition (Figure $5.7\left(\mathbf{F}-\mathbf{F}^{\prime}\right)\left(\mathbf{G}-\mathbf{G}^{\prime}\right)\left(\mathbf{H}-\mathbf{H}^{\prime}\right)\left(\mathbf{I}-\mathbf{I}^{\prime}\right)$ $\left.\left(\mathrm{J}-\mathrm{J}^{\prime}\right)\right)$.

\subsubsection{SMAD2/3 Regulate Key Genes Involved in Adipogenesis, Lipogenesis and Lipolysis}

To further investigate the causes of the reduced fat depot sizes of Smad2/3cKO mice, we examined the mRNA levels of adipogenic, lipogenic and lipolytic markers from subcutaneous WAT from Smad2/3cKO female mice. Smad2/3cKO mice showed significantly reduced levels of Smad 2 and Smad3 transcripts, as expected, on both LFD and HFD. Under LFD conditions, adipogenic markers, Cebp $\alpha, C e b p \beta, P p a r \gamma$, and Pref1 were decreased in Smad2/3cKO but did not reach significance. Conversly Fabp4 and Wnt10b increased but not significantly under LFD conditions (Figure 5.8 A). In contrast, on HFD conditions, adipogenic markers Cebp $\alpha$, Ppar $\gamma$ and Wnt10b decreased significantly, while Cebp $\beta$ increased but not significantly. Fabp4 and Pref1 decreased but did not reach significance in Smad2/3cKO mice (Figure 5.8 B).

On HFD, genotype effect was observed for several gene involved in lipogenesis and lipolysis. Smad2/3cKO females on LFD showed no significant changes in these genes 
Acaca, while Fasn and Perilipin were lower, Dgat2 and Cd36 were slightly higher although these genes did not reach statistical significance (Figure 5.8 C). On HFD conditions, however, lipogenic and lipolytic markers, Fasn, Dgat2, Cd36, Hsl and Scd1 were significantly downregulated while we found reduced expression with Acaca, Perilipin and Atgl, but did not reach significance (Figure 5.8 D). Taken together, these data suggest a possible cellular mechanism by which adipose selective SMAD2/3 deletion results in the reduced adiposity of $S m a d 2 / 3 \mathrm{cKO}$ female mice under HFD conditions.

\subsection{Discussion}

Using adipose selective knockout of $S m a d 2 / 3$, we have shown that adipose tissue-selective reduction of Smad2/3 using Adiponectin-Cre had sex-selective effects on diet-induced weight gain. Smad2/3cKO male mice had similar body weight compared to controls irrespective of diet, while Smad2/3cKO female mice gained less weight on HFD, exhibiting less visceral and subcutaneous fat mass. Associated with this phenomenon is the downregulation of genes involved in adipogenesis, lipogenesis and lipolysis, reduced hepatic steatosis and increased metabolic rate. This demonstrates that SMAD2 and SMAD3 have partially overlapping roles in adipose tissue development or function such as with adipose selective deletion, (1) Smad2 showed no phenotype, (2) Smad3 showed mild phenotype in males and (3) Smad2/3 showed strong phenotype in females. We speculate that the differences in outcomes between males and females may be linked to sex hormone responses and endocrine mediated pathways. Taken together, this study highlights that sex is an important factor to consider when assessing phenotypes observed with Smad3 and in Smad2/3 mediated signal transduction.

It is important to acknowledge a potential limitation of this study, which was our decision to use the Adiponectin-Cre mouse as stated in chapter 4. To achieve adiposeselective gene inactivation, and for studying events that occur in the early to middle stages of adipogenesis, it will be very informative to use and dissect the role of SMAD2/3 using Cre expressed at a stages earlier than Adiponectin-Cre during embryogenesis. It is plausible that SMAD-mediated events occurring at earlier stages of adipogenesis would have been missed as a consequence of the timing of SMAD2/3 inactivation. Thus, to fully understand the role of SMAD-mediated signaling and associated molecular mechanisms that occur during earlier stages, additional studies are needed using alternative strategies with early Cre expressing in preadipocytes. This may allow us to investigate roles of SMAD2/3 at specific time points to better understand the relationship between SMAD2, SMAD3, adipocyte development and function. Also, investigating the mechanism by which Smad2/3 play a role in modulating sex selective phenotypes is an important future direction. 


\section{Chapter 6}

\section{Role of Activins on Preadipocyte Proliferation, Differentiation and Cell Fate}

\subsection{Introduction}

Activin signaling is known to regulate cell proliferation in many contexts including adipose tissue. Adipose tissue specific loss of activin A and B results in severe reduction of adipose tissue mass suggesting that activins are essential for the specification, differentiation and maintenance of proper adipose tissue growth (Namwanje and Irudayam et al. in preparation). SMAD-mediated activin signaling regulates cell proliferation. Activin A promotes proliferation in 3T3-L1 preadipocytes and adipocyte progenitors (Hirai et al., 2005; Zaragosi et al., 2010). However, the cell autonomous roles of activins on cell proliferation in primary preadipocytes isolated from visceral and subcutaneous depots had not been assessed. Therefore, we examined the role of activin A, activin receptor inhibitor, SB431542 and SMAD3 specific inhibitor, SIS3 in proliferating preadipocytes isolated from subcutaneous depots.

Additionally, it has been shown that activin A inhibits differentiation via SMAD2 in 3T3-L1 and human adipocyte progenitors (Hirai et al., 2005; Zaragosi et al., 2010). However, the cell autonomous roles of activins on cell differentiation and cell fate in primary culture cells had not been assessed. Additionally, in previous studies from our lab, it has been observed that the loss of activins A and B alters the phenotypic characteristics of white adipose tissue (WAT) in mice, including smaller adipocytes and histologic features typically associated with brown adipose tissue (BAT) (Namwanje and Irudayam et al. in preparation).

To determine the effects of activins on adipocyte differentiation, we first determined the cell autonomous role of activins on adipogenesis and cell fate using mouse embryo fibroblasts (MEFs). We compared WT and activin A/activin B double knockout (Inhbb-/-, Inhba-/-). As activin double knockout (here in activin DKO) mice die on postnatal day 1. Therefore, we developed a cell-based assay by isolating MEFs from WT and activin DKO embryos at e13.5 and differentiated them in vitro. After getting activin DKO embryos, which was challenging due to limited numbers of the desired genotype, we did several rounds of experiments at different times, encompassing four biological replicates of WT and activin 

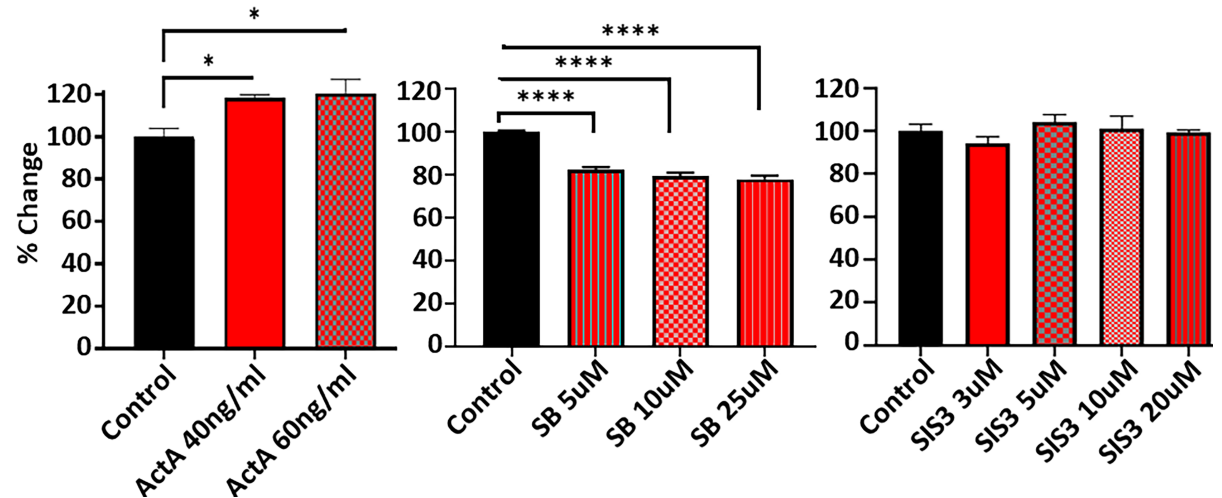

Figure 6.1: Activin A Promotes Subcutaneous Preadipocyte Proliferation While Activin Receptor Inhibitor SB431542 Inhibits Proliferation.

Proliferation of preadipocytes in presence or absense of activin A (40ng/mL or $60 \mathrm{ng} / \mathrm{mL})$ (left), activin receptor inhibitor, SB431542 $(5 \mu \mathrm{M}, 10 \mu \mathrm{M}$, or $25 \mu \mathrm{M})$ (middle) and SMAD3-specific inhibitor, SIS3 $(3 \mu \mathrm{M}, 5 \mu \mathrm{M}, 10 \mu \mathrm{M}$ or $20 \mu \mathrm{M}$ )(right) for $48 \mathrm{hrs}$. Student's T-test was applied with p value $\leq 0.05$ considered as statistically significant for each experiment. ${ }^{*} \mathrm{p}<0.05,{ }^{* * * *} \mathrm{p}<$ 0.00005 .

DKO. MEF isolation, differentiation, bioenergetics, RNA, and protein analyses were all performed on all biological replicates.

\subsection{Results}

\subsubsection{Activin A Promotes Proliferation of Preadipocytes}

To determine the effect of activin A on preadipocyte proliferation, subcutaneous preadipocytes from wild type mice were treated with or without activin A $(40 \mathrm{ng} / \mathrm{mL}$ or $60 \mathrm{ng} / \mathrm{mL}$ ) (left), activin receptor inhibitor, SB431542 $(5 \mu \mathrm{M}, 10 \mu \mathrm{M}$, or $25 \mu \mathrm{M})$ (middle), and SMAD3-specific inhibitor, SIS3 $(3 \mu \mathrm{M}, 5 \mu \mathrm{M}, 10 \mu \mathrm{M}$ or $20 \mu \mathrm{M})$ (right) in $10 \%$ growth medium every $24 \mathrm{hrs}$ for 2 days. Viability was assessed by MTT assay following manufacturer's instructions. A dose dependent effect of activin A was observed on proliferation. Activin A treatment augmented proliferation while SB431542 inhibited proliferation in a dose dependent manner (Figure 6.1 ). SMAD3-specific inhibitor, SIS3 had no effect on cell proliferation at any concentration. During treatment with activin, SB and SIS3, we did not observe cell death, assayed using flow cytometry by annexin-V staining (Figure 6.2 ). 


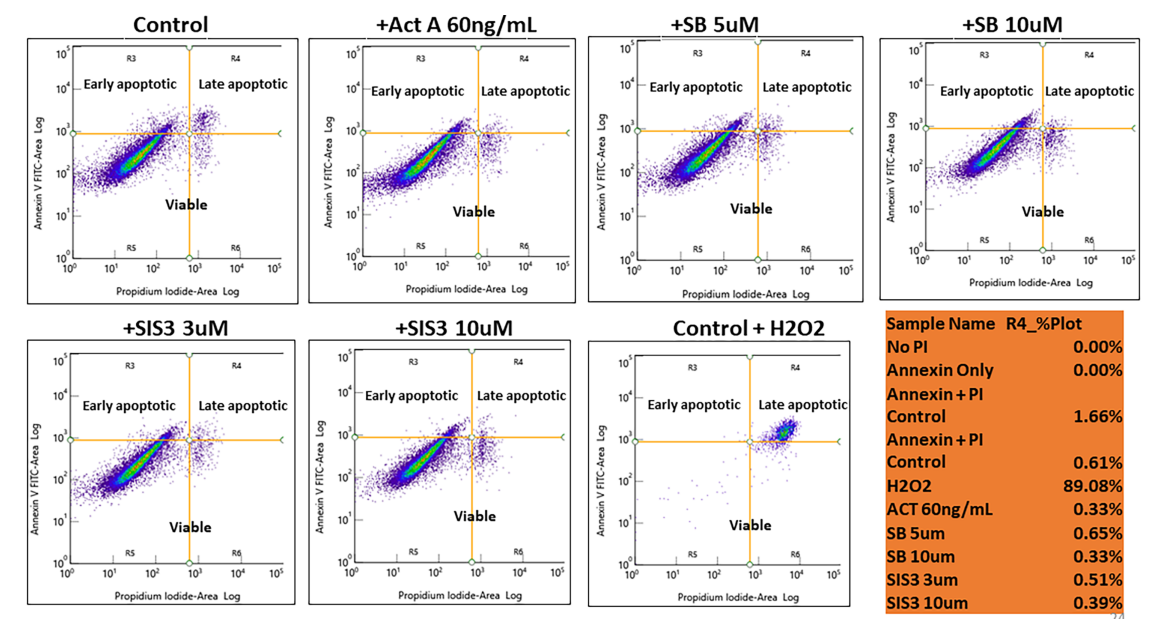

Figure 6.2: Apoptosis Assay. Annexin-V Staining showing no apoptotic cells in activin A, SB431542 and SIS3-treated preadipocytes, while cells treated with $\mathrm{H} 2 \mathrm{O} 2$ showed very high level of apoptotic cells served as a positive control.

\subsubsection{Activin Deficiency Promotes Adipogenesis in Differentiating MEFs}

To determine the consequences of total loss of activin A and activin B on differentiating activin DKO cells. We examined the expression of adipogenic markers in early and late stages of differentiation. MEFs were induced with hormonal cocktail and harvested at the indicated time points (day3,5 and 8). Activin deficiency promoted faster differentiation of MEFs, as determined by morphological appearance (phase contrast, Oil Red O, BODIPY and DAPI staining) (Figure 6.3 top). This was further demonstrated by adipogenic marker mRNA expression in the differentiating MEFs (WT vs activin DKO). Interestingly, the expression of Cebp $\alpha, C e b p \beta$ and Ppar $\gamma$ was increased on days 3, 5 and 8 with significant increases of all 3 genes on day 8. The expression of Cebp $\delta$ did not change much at any time point and Fabp 4 was significantly increased on day 3 but did not show significant differences on days 5 and 8. Preadipocyte specific markers Pref1 and Wnt10b did not change significantly. Therefore, the combined deficiency of activin A and activin B resulted in enhanced adipogenesis (Figure 6.3 bottom left). Because of the smaller sizes of adipocytes observed in activin-deficient mice, we measured glycerol released in the media of differentiated activin DKO and WT MEFs to determine if lipolysis might be a contributing factor. The results showed a significant increase in glycerol release in activin DKO adipocytes which further increased in cells treated with $(5 \mu \mathrm{M})$ isoproterenol, however similar effects were observed in both genotypes (Figure 6.3 bottom right). 

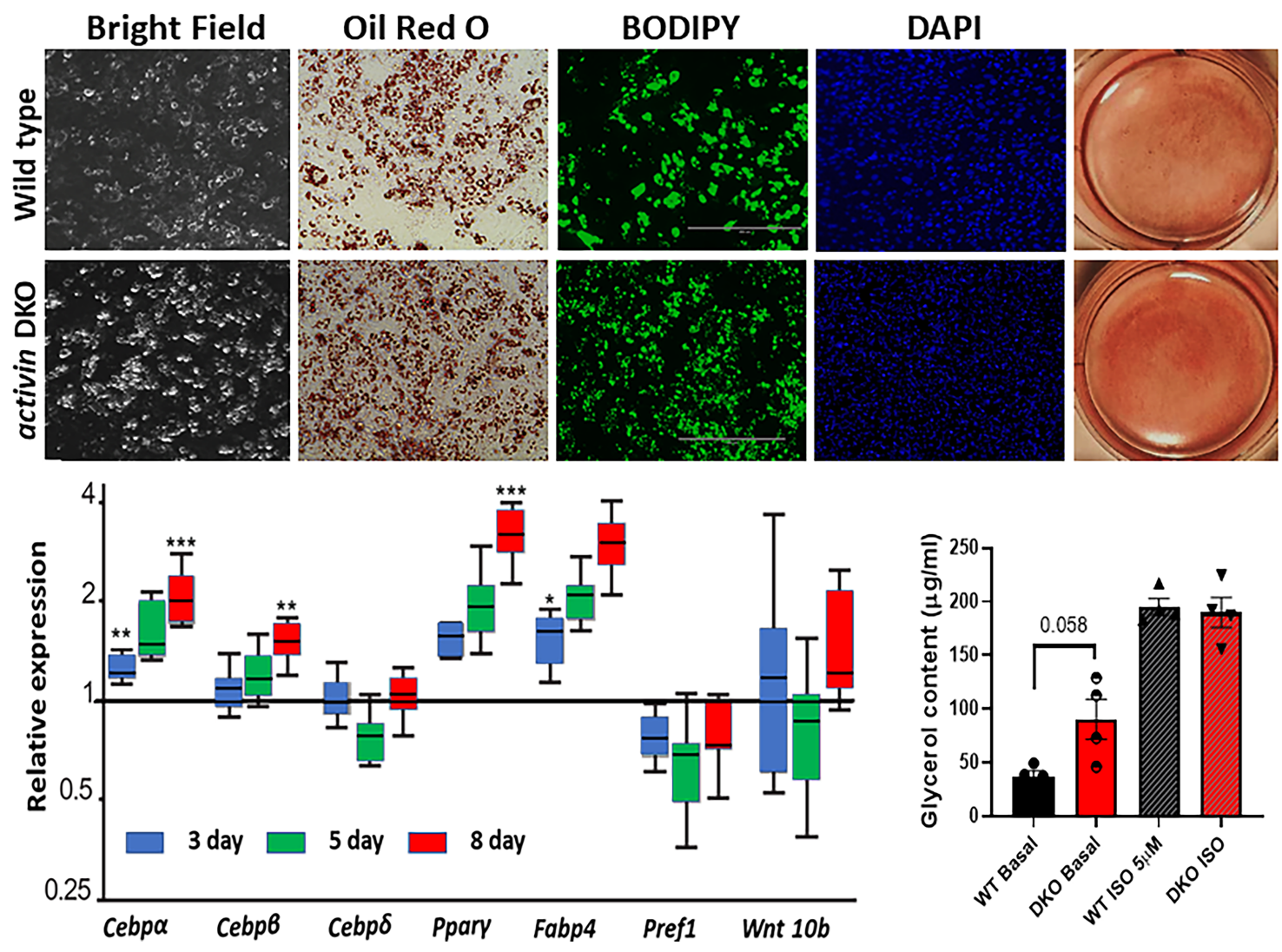

Figure 6.3: Activin Deficiency Promotes Adipocyte Differentiation of MEFs. Phase contrast, Oil Red O, BODIPY and DAPI staining showing morphology of WT and activin DKO MEFs differentiated into adipocytes on day 8 (top). Graph shows the relative gene expression of adipocyte differentiation markers evaluated by RT-PCR on day 3, 5 and 8 of differentiation. ${ }^{*} \mathrm{p}<0.05$; ${ }^{* *} \mathrm{p}<0.005 ;{ }^{* * *} \mathrm{p}<0.001$, ( $\mathrm{n}=3$ embryo per genotype) (Bottom left). Cyclophilin was used as a normalizer. Quantitative enzymatic determination of glycerol showed a significant level of glycerol release in activin DKO cell which further increases in the ISO $(5 u \mathrm{M})$ treated cells (Bottom right). 


\subsubsection{Activin Deficiency Induces Browning in Differentiating Activin DKO MEFs}

To ascertain the bioenergetic potential of activin deficient MEFs, we examined the brown adipocyte-specific markers. We first looked at expression by immunofluorescence of the browning marker, UCP1 in activin DKO and WT differentiated MEFs. Activin DKO cells demonstrated significantly more UCP1 positive cells than WT (Figure 6.4 top left). Browning markers Ucp1, Cidea, Pgc1 $\alpha$ and Prdm16 were examined on days 3, 5 and 8 in the presence or absence of isoproterenol (Figure 6.4 top right). The qRT-PCR analysis showed significant upregulation of brown markers, Ucp 1 and Cidea as early as day 3 and remained significantly increased on days 5 and 8 , while $P g c 1 \alpha$ was significantly increased on days 5 and 8 , when most of the cells were mature adipocytes. We did not see changes in Prdm16 (a very early marker of brown adipogenesis) (Figure 6.4 bottom left). Further, addition of isoproterenol (ISO), had no further effect on gene expression other than a modest, significant reduction in Prdm16 (Figure 6.4 bottom right).

\subsubsection{Enhanced Mitochondrial Gene Expression in Activin-Deficient Differen- tiated MEFs}

We further looked at several browning and other markers involved in mitochondrial biogenesis using antibodies against UCP1, PGC-1 $\alpha$ and electron transport chain (ETC) complexes. We found that UCP1 and PGC-1 $\alpha$ were significantly elevated in activin DKO differentiated MEFs on days 5 and 8 (5-day data not shown) (Figure 6.5 top and bottom). We further examined the expression of ETC components from complexes I, II, III, IV and V on days 5 and 8. OXPHOS antibody cocktail directed against ETC complex proteins showed a significantly elevated levels in activin DKO cells on day 5 and 8 (5-day data not shown) (Figure 6.5 top and bottom). This is yet another indication that cell autonomous effects of activin deficiency are linked to a bioenergetic profile that is consistent with a "brown-like" adipocyte phenotype. Increased mitochondrial number and activity in differentiated activin DKO MEFs has been corroborated by Seahorse and Mitotracker analysis (data not shown, Namwanje and Irudayam et al. in preparation).

Thus, the expression of UCP1, PGC- $1 \alpha$ and ETC complex proteins showed that the mitochondria of activin DKO cells are more abundant than WT to meet the higher energy demands of the observed "brown" like cells.

The observed browning in differentiated, "activin-deficient" MEFs prompted us to investigate whole transcriptional changes. We isolated total RNA from differentiated (day 8) MEFs and performed RNA-seq. This analysis showed 3179 differentially expressed genes at day 8 of differentiation in activin DKO cells when compared to WT cells $(\mathrm{P}<0.05$, fold change $>1.5)$. Among differentially expressed genes, 239 were involved in lipid metabolic processes (Figure 6.6 circle bar in left). 

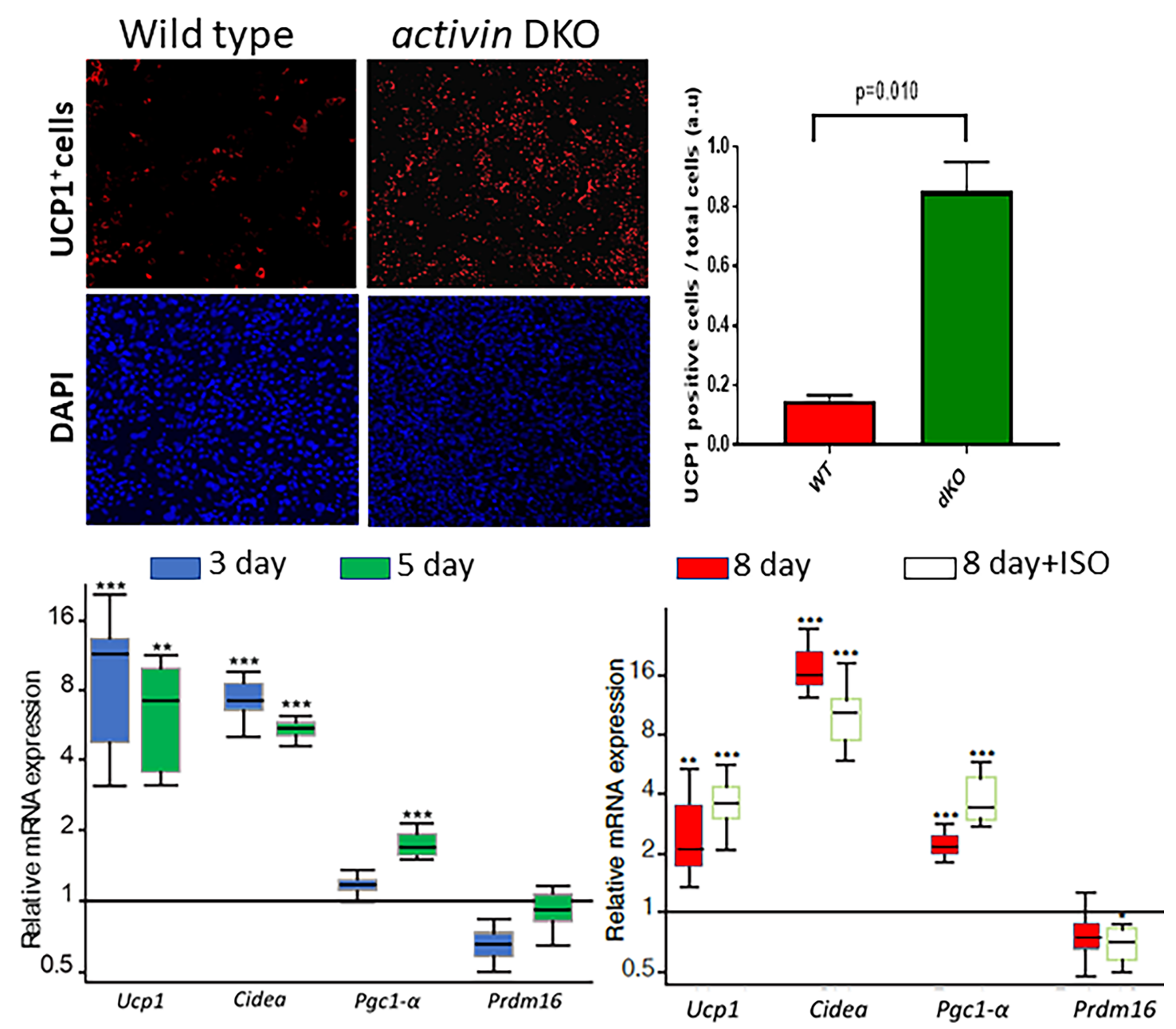

Figure 6.4: Activin Deficiency Induces Browning in MEFs.

Immunofluorescence staining for UCP+ cells (top left) and quantitation ${ }^{*} \mathrm{p}$ $<0.01$ ) (top right) in differentiated MEFs on day 8 isolated from WT and activin DKO MEFs. RT-PCR showing activin DKO MEF exhibit enhanced expression of BAT-specific genes (Ucp1, Cidea, Pgc1- $\alpha$ and Prdm16) on days 3, 5 and 8 of differentiation (bottom). $5 \mathrm{uM}$ of Isoproterenol treatment shows no additionally significant effect on BAT specific genes (bottom right). mRNA fold difference relative to normalizer (Cyclophilin). ${ }^{*} \mathrm{p}<0.05$; ${ }^{* *} \mathrm{p}<0.005$; ${ }^{* * *} \mathrm{p}<0.001$. ${ }^{*} \mathrm{P}<0.05$, considered statistically significant relative to wild type ( $\mathrm{n}=3$ embryo per genotype). 


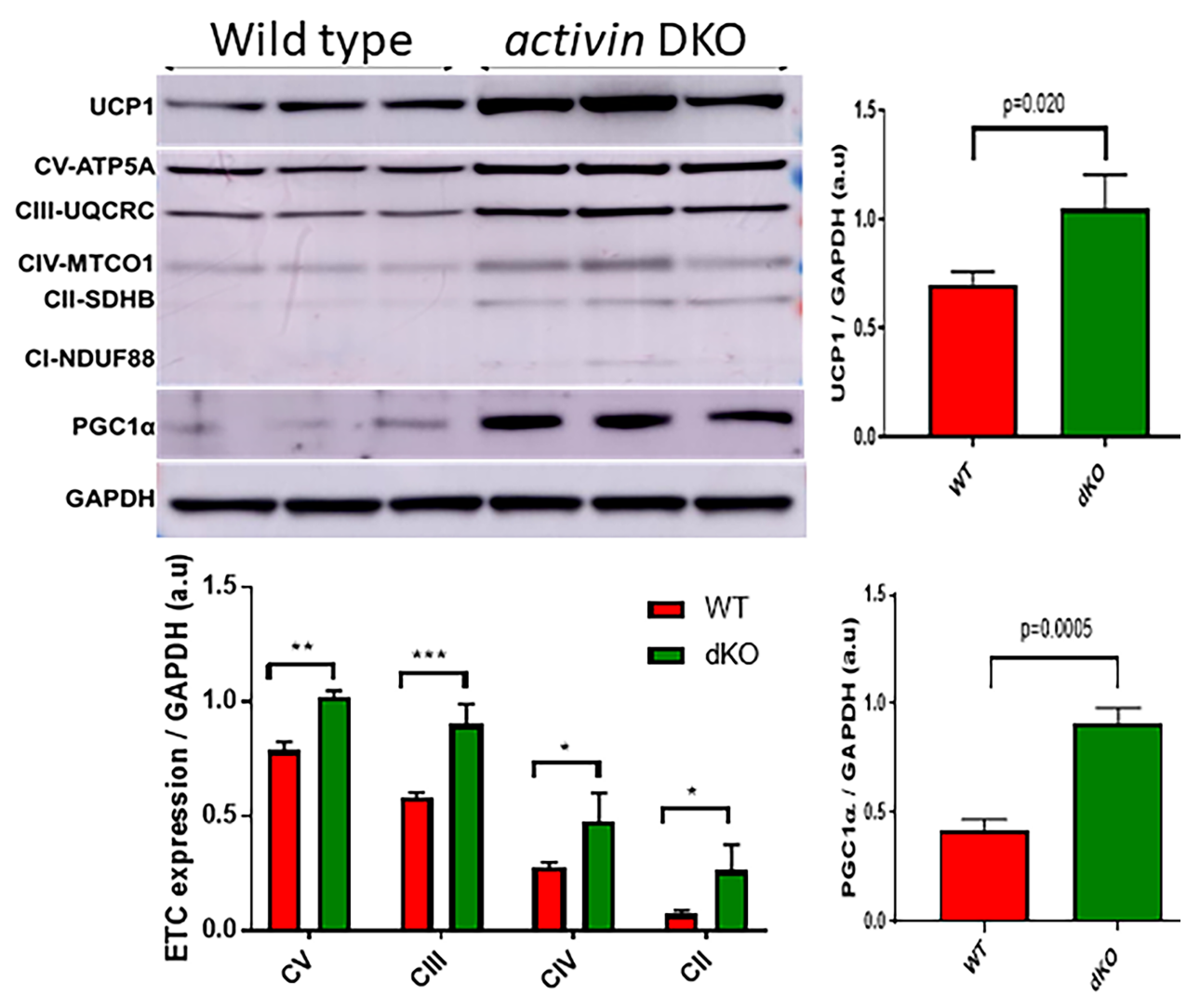

Figure 6.5: Enhanced Mitochondrial Gene Expression in

Activin-Deficient Differentiated MEFs. Western blot analysis of UCP1, PGC1 $\alpha$ and Electron transport chain complex (ETC) indicates increased level of protein in activin DKO cells relative to WT cells ((top left). Quantitation of UCP1, ETC protein and PGC1 $\alpha$ (top right and bottom). Band intensity quantitation of UCP1, ETC protein and PGC1 $\alpha$ compared to GAPDH in image J. *p $<0.05 ;{ }^{* *} \mathrm{p}<0.005 ;{ }^{* * *} \mathrm{p}<0.001 .{ }^{*} \mathrm{P}<0.05$, considered statistically significant relative to wild type ( $\mathrm{n}=3$ embryo per genotype). 


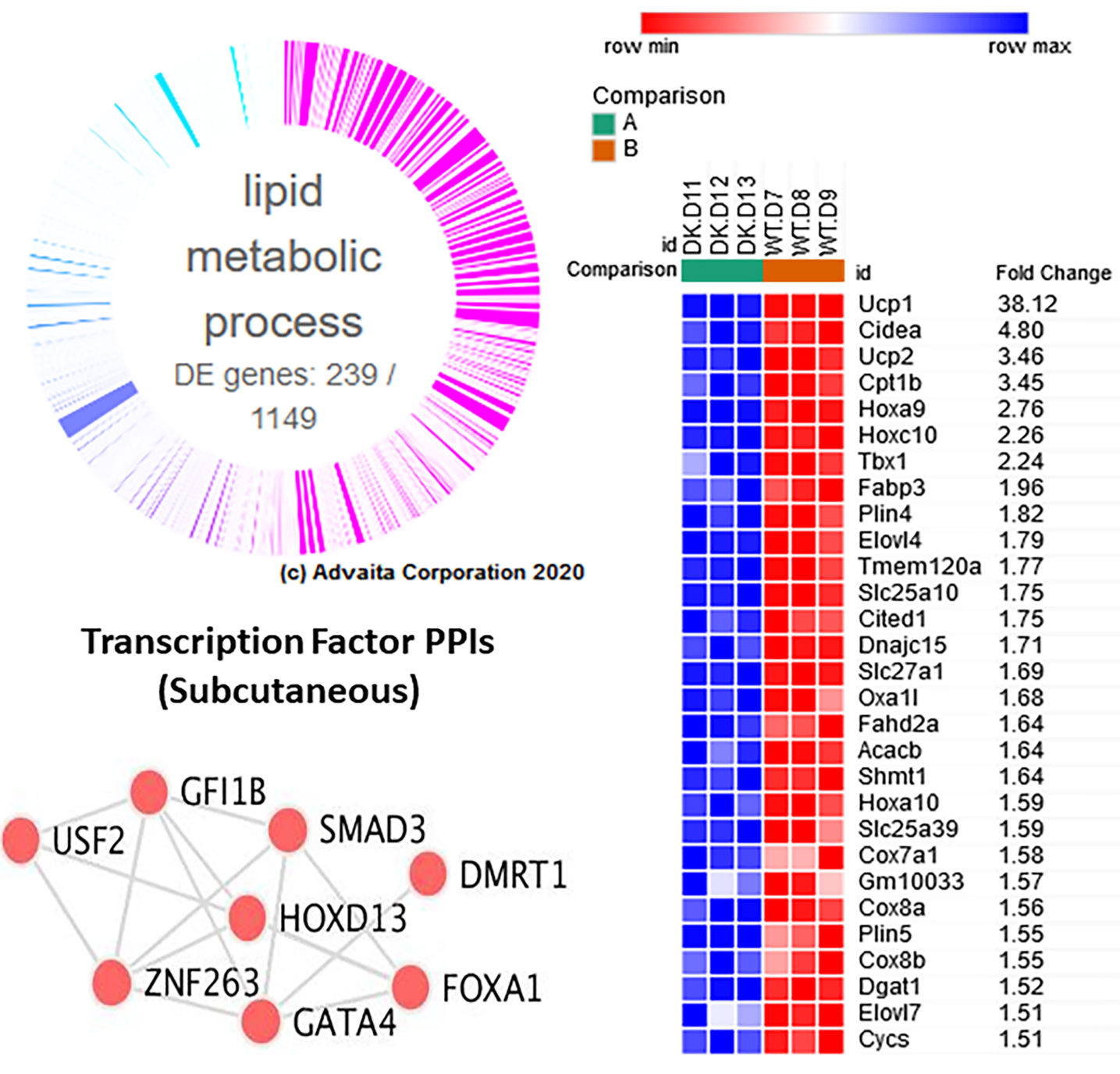

Figure 6.6: Activin Deficiency Induces Browning Genes in MEFs.

Differentially expressed genes involved in lipid metabolism (circle bar in left), heat map of RNA-seq showing signature genes associated with browning with fold changes (right) and protein-protein interaction map showing key transcription factors involved in adipogenesis (bottom left). 


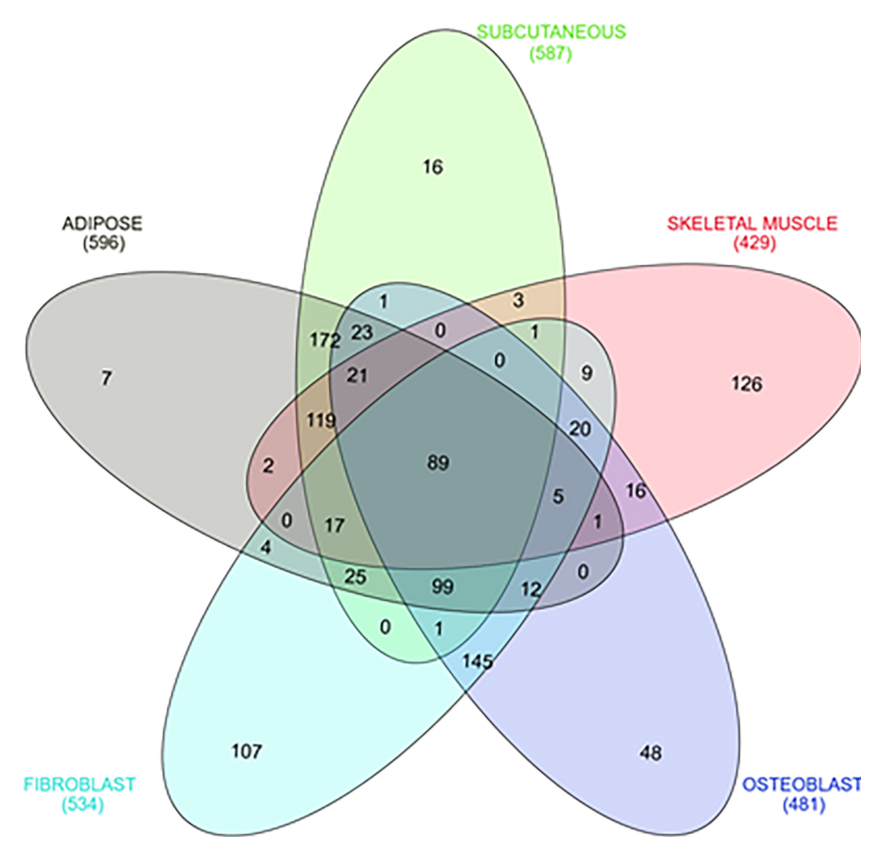

Figure 6.7: Differentially Expressed Genes Share Common Ancestral Lineages. Venn diagram shows differentially expressed genes in activin DKO differentiated MEFs. These genes share signature involved in the differentiation of different tissue types.

Additinally, upregulation of browning and mitochondrial gene signatures were observed, corroborating previous results. Activin-deficient adipocytes exhibited significant upregulation in transcripts involved in browning, mitochondrial function, and energy expenditure including Ucp1, Cidea and several others (Figure 6.6 heat map, right). We also observed enrichment of several transcription factors involved in adipogenesis, including SMAD3 (Figure 6.6 bottom left).

Thus, total activin loss has resulted in an increase of bioenergetic markers at the mRNA and protein levels, endorsing that the activin DKO cells are assuming brownlike characteristics at early stages of differentiation. Additionally, hierarchical clustering revealed a gene signature related to skeletal muscle, osteoblasts, fibroblasts, consistent with the ancestral link between fat, osteoblasts and skeletal muscle (Figure 6.7 ) and (Figure 2.1)

We further analyzed differentially expressed genes using EnrichR. The KEGG 2019 Mouse Pathways identified several key signaling pathways that are involved in adipose tissue growth, survival, and energy homeostasis for the differentially expressed genes in activin DKO differentiated MEFs compared to WT MEFs. Among top signaling pathways, PI3K-AKT signaling was the first hit among the significantly enriched pathways, a key 
pathway dysregulated in obesity and diabetes (X. Huang et al., 2018) (Table 6.1).

Table 6.1: KEGG 2019 Mouse Pathways Analysis.

\begin{tabular}{|c|c|c|c|c|}
\hline Name & P-value & $\begin{array}{l}\text { Adjusted } \\
\text { P-value }\end{array}$ & $\begin{array}{l}\text { Odds } \\
\text { Ratio }\end{array}$ & $\begin{array}{c}\text { Combined } \\
\text { Score }\end{array}$ \\
\hline PI3K-Akt signaling pathway & $1.7 \mathrm{E}-10$ & 5.1E-08 & 3.327 & 74.90 \\
\hline Focal adhesion & 3.3E-09 & 5.0E-07 & 4.033 & 78.73 \\
\hline $\begin{array}{l}\text { Cytokine-cytokine receptor } \\
\text { interaction }\end{array}$ & $1.5 \mathrm{E}-07$ & $9.1 \mathrm{E}-06$ & 3.078 & 48.38 \\
\hline $\begin{array}{l}\text { Regulation of lipolysis in } \\
\text { adipocytes }\end{array}$ & $7.8 \mathrm{E}-06$ & $3.4 \mathrm{E}-04$ & 5.733 & 67.41 \\
\hline $\begin{array}{l}\text { Hypertrophic cardiomyopathy } \\
\text { (HCM) }\end{array}$ & 7.2E-05 & $2.4 \mathrm{E}-03$ & 4.106 & 39.16 \\
\hline MAPK signaling pathway & $1.5 \mathrm{E}-04$ & $3.4 \mathrm{E}-03$ & 2.402 & 21.22 \\
\hline $\begin{array}{l}\text { Cell adhesion molecules } \\
\text { (CAMs) }\end{array}$ & $9.0 \mathrm{E}-04$ & $1.5 \mathrm{E}-02$ & 2.644 & 18.53 \\
\hline $\begin{array}{l}\text { Regulation of actin } \\
\text { cytoskeleton }\end{array}$ & $3.4 \mathrm{E}-03$ & 4.3E-02 & 2.219 & 12.61 \\
\hline TNF signaling pathway & 7.4E-03 & $6.6 \mathrm{E}-02$ & 2.627 & 12.87 \\
\hline Insulin resistance & 7.4E-03 & $6.8 \mathrm{E}-02$ & 2.627 & 12.87 \\
\hline PPAR signaling pathway & 1.7E-02 & $1.2 \mathrm{E}-01$ & 2.644 & 10.81 \\
\hline Estrogen signaling pathway & $2.4 \mathrm{E}-02$ & 1.6E-01 & 2.156 & 8.00 \\
\hline Osteoclast differentiation & 4.7E-02 & 2.7E-01 & 2.006 & 6.13 \\
\hline TGF-beta signaling pathway & $6.5 \mathrm{E}-02$ & 3.6E-01 & 2.117 & 5.80 \\
\hline Insulin signaling pathway & $6.9 \mathrm{E}-02$ & 3.6E-01 & 1.848 & 4.93 \\
\hline Glycolysis / Gluconeogenesis & $1.6 \mathrm{E}-01$ & $6.0 \mathrm{E}-01$ & 1.917 & 3.56 \\
\hline $\begin{array}{l}\text { Adipocytokine signaling } \\
\text { pathway }\end{array}$ & $1.8 \mathrm{E}-01$ & $6.5 \mathrm{E}-01$ & 1.809 & 3.10 \\
\hline Type II diabetes mellitus & 1.9E-01 & $6.5 \mathrm{E}-01$ & 2.006 & 3.36 \\
\hline $\begin{array}{l}\text { Phospholipase D signaling } \\
\text { pathway }\end{array}$ & $1.8 \mathrm{E}-01$ & 6.6E-01 & 1.508 & 2.55 \\
\hline $\begin{array}{l}\text { Inflammatory mediator } \\
\text { regulation of TRP channels }\end{array}$ & 2.0E-01 & 7.0E-01 & 1.517 & 2.40 \\
\hline Drug metabolism & $2.8 \mathrm{E}-01$ & 8.6E-01 & 1.408 & 1.78 \\
\hline Pyruvate metabolism & 3.3E-01 & 9.4E-01 & 1.690 & 1.86 \\
\hline Fatty acid biosynthesis & 3.6E-01 & $9.6 \mathrm{E}-01$ & 2.293 & 2.36 \\
\hline p53 signaling pathway & 3.8E-01 & 9.9E-01 & 1.356 & 1.31 \\
\hline Thermogenesis & 4.3E-01 & $1.0 \mathrm{E}+00$ & 1.112 & 0.94 \\
\hline FoxO signaling pathway & 5.9E-01 & $1.0 \mathrm{E}+00$ & 0.973 & 0.51 \\
\hline Th17 cell differentiation & $6.2 \mathrm{E}-01$ & $1.0 \mathrm{E}+00$ & 0.944 & 0.45 \\
\hline
\end{tabular}


Table 6.1 Continued from Previous Page.

\begin{tabular}{lcccc}
\hline Name & P-value & $\begin{array}{c}\text { Adjusted } \\
\text { P-value }\end{array}$ & $\begin{array}{c}\text { Odds } \\
\text { Ratio }\end{array}$ & $\begin{array}{c}\text { Combined } \\
\text { Score }\end{array}$ \\
\hline $\begin{array}{l}\text { Signaling pathways regulating } \\
\text { pluripotency of stem cells }\end{array}$ & $6.2 \mathrm{E}-01$ & $1.0 \mathrm{E}+00$ & 0.937 & 0.45 \\
\begin{tabular}{l} 
Citrate cycle (TCA cycle) \\
\hline
\end{tabular} & $6.4 \mathrm{E}-01$ & $1.0 \mathrm{E}+00$ & 1.003 & 0.45 \\
\hline
\end{tabular}

PI3K-AKT signaling affects many pathways involved in energy homeostasis, adipogenesis and metabolism, including glycolysis, gluconeogenesis, fatty acid oxidation and cell cycle progression (Figure 6.8).

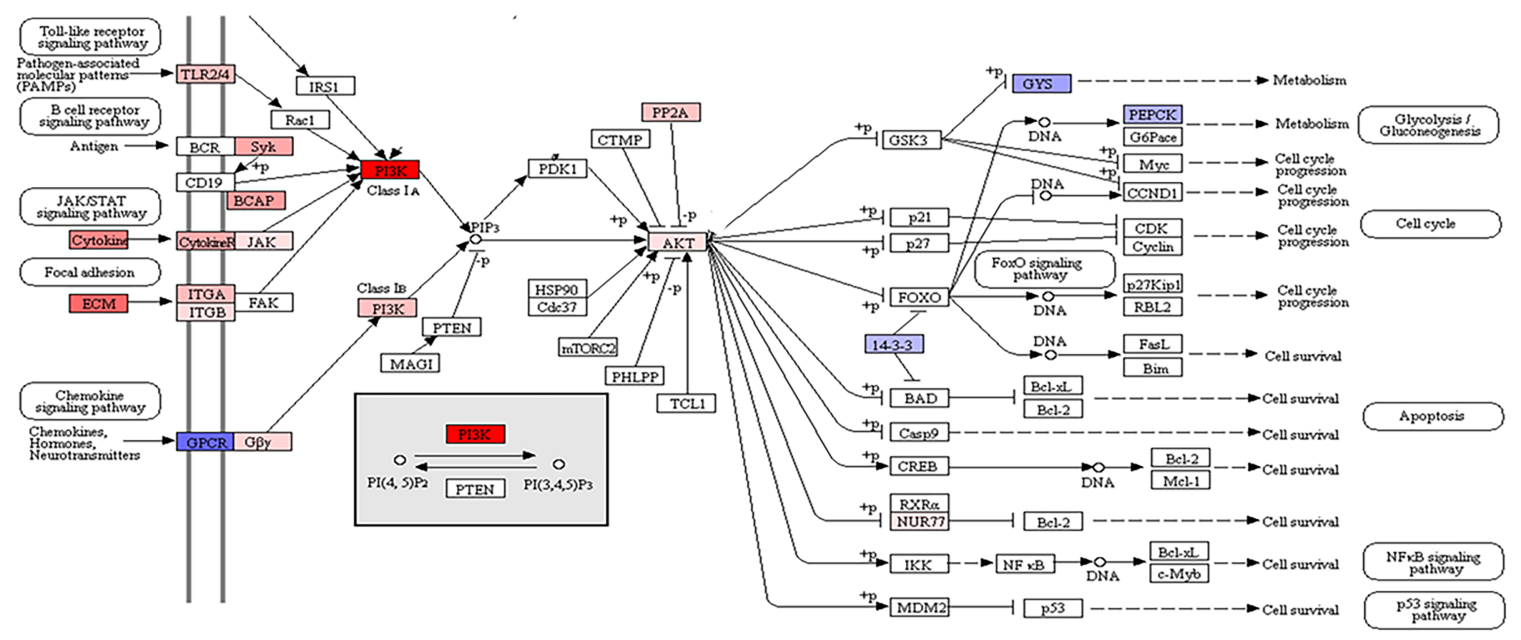

Figure 6.8: PI3K-AKT Signaling Pathway.

Further, I validated transcription factors in the PI3K-AKT pathway by western blot. pFOXO1/3 was significantly increased in activin DKO in undifferentiated and differentiated MEFs suggesting that this transcription factor might play important roles at both stages (undifferentiated and differentiated) (Figure 6.9). No change in the protein level was observed for total FOXO1, pMTOR and pERK1/2. FOXO1/FOXO3 are required for proliferation. Phosphorylated FOXO1/FOXO3 are excluded from nucleus and restrained in the cytoplasm, thereby inhibiting proliferation. This phenotype is consistent with in vivo data that activin deficiency in mice leads to arrested development of adipose tissue and display markedly reduced adiposity (data not shown, Namwanje and Irudayam et al. in preparation). 


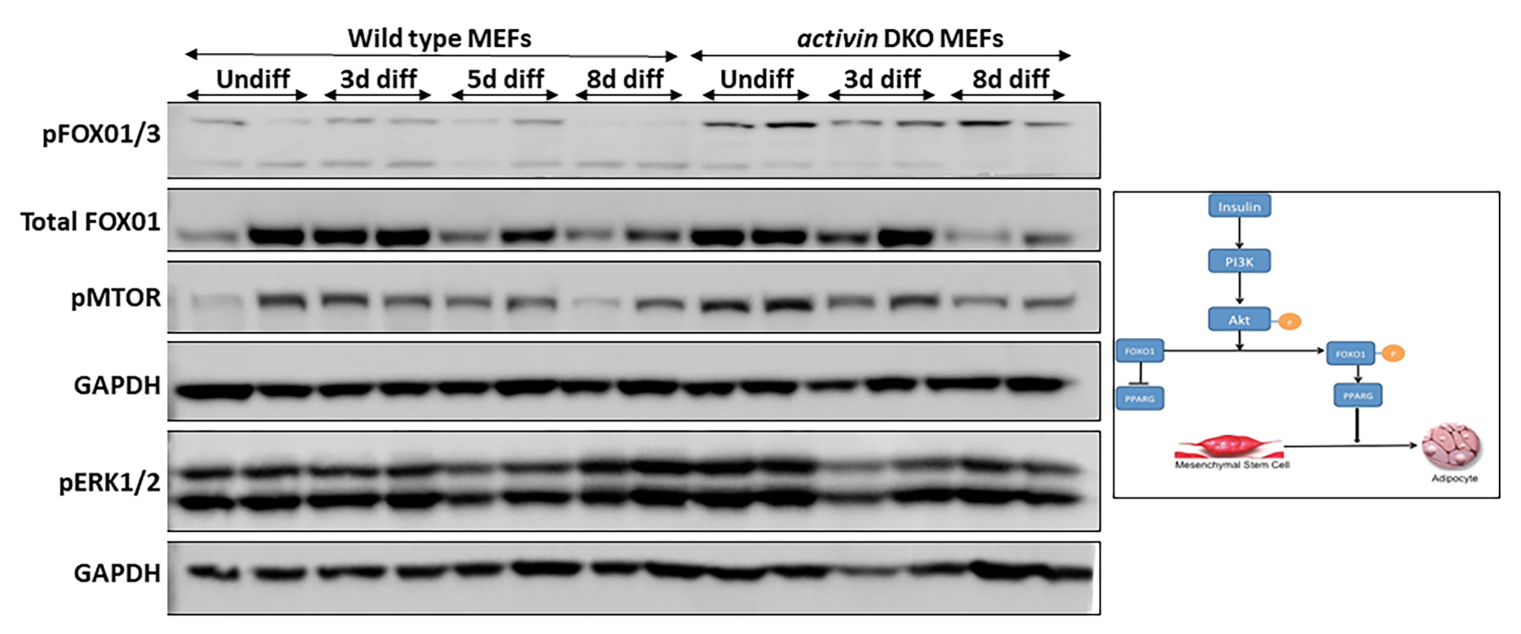

Figure 6.9: Validation of Key Transcription Factors in Activin DKO and WT MEFs. Western blot analysis showing of changes in the phosphorylation of FOXO1/3 protein in differentiated and undifferentiated MEFs, total FOXO1, pMTOR, PERK1/2 and GAPDH in activin DKO MEFs relative to WT MEFs

\subsection{Discussion}

\subsubsection{Early Stage of Browning in Activin DKO Adipocytes Is Intrinsic and Cell Autonomous Due to Complete Loss of Activin A and Activin B}

Activin signaling is a key pathways that regulates the expression of genes involved in preadipocyte proliferation and subsequent differentiation into mature adipocytes for the maintenance of adipose tissue mass (Zaragosi et al., 2010). Total loss of activin A and activin $B$ promotes differentiation of MEFs into adipocytes with brown-like characteristics as early as day 3 (early stage differentiation) including abundant mitochondria with increased ETC complex protein directly correlating with enhanced mitochondrial biogenesis and abundant respiratory chain proteins. The expression of UCP1, PGC- $1 \alpha$ and ETC complex proteins suggest that the mitochondria of activin DKO cells are well equipped to meet a higher energy demand than WT cells. In particular, the mitochondrial enrichment through upregulation ETC complex proteins point out with no ambiguity that these effects are uniquely connected to cell autonomous total loss of activin A and activin B. Total loss of activins A and activin $B$ significantly induced the expression of brown-like markers in differentiated MEFs. It is important to note that the up regulation of browning markers is consistent with in vivo data that the combined deficiency of activin A and activin B induced multiloculated beige/brown-like cells in visceral WAT. SMAD2/3 are known downstream transcriptional mediators of activin signaling, and whole transcriptome analysis revealed that in addition 
to SMAD2/3, FOXO1/FOXO3 of the PI3K-AKT pathway and PPAR $\gamma$ may also be key transcriptional regulators of activin signaling during adipogenesis. In conclusion, whole transcriptome analysis opens the way for the analysis of crosstalk between several signaling pathways and associated downstream events for the regulation of genome organization and WAT gene expression. These data are consistent with important roles for activins, SMAD2/3, activin and crosstalk with additional metabolic signaling pathways including PI3K-AKT, in the control of adipocyte proliferation and differentiation including adipocyte fate decisions. Dysregulation of these processes has provided insight into mechanisms by which beneficial metabolic effects, such as browning of WAT or protection from diet-induced obesity might be achieved. 


\section{Chapter 7}

\section{Conclusion and Significance}

\subsection{Conclusion}

Activin A and activin B are TGF- $\beta$ family members that play prominent roles in WAT. We have shown that reduced activin signaling results in reduced adiposity and the appearance of brown like cells in WAT in vivo, however the downstream molecular mechanism that contribute to this phenotype remained unknown. The overall aim of this dissertation was to investigate the role of activin/ SMAD2 and SMAD3 signaling on adipose tissue function and the molecular mechanism(s) by which activin signaling regulates adipocyte differentiation and cell fate using cellular approaches and mouse models. To examine the cell autonomous roles of activin on cell proliferation, differentiation, and cell fate and the contributions of downstream transcriptional transducers SMAD2/3 to these processes. This study gave an additional insight into understanding the roles of activin/SMADs in the regulation of adiposity.

The first aim of my dissertation included mouse model studies. Using these models, we have demonstrated the roles of SMAD2 and SMAD3 on adipose tissue development and function. We have shown that SMAD2 and SMAD3 proteins exhibit different temporal expression during adipose tissue development confirmed in both visceral and subcutaneous WAT. Adipose selective deletion of $S m a d 2$ had no role on adipose tissue development while adipose-selective deletion of Smad3 partially protected the male mice from DIO. Smad3 deletion in male mice resulted in reduced adiposity in subcutaneous WAT as demonstrated by smaller body weight, smaller depot and adipocyte sizes and by molecular markers in subcutaneous fat depot. Furthermore, these findings support our hypothesis that SMAD3 functions in a depot-selective manner for the development of adipose tissue (Chapter 4).

The second aim of my dissertation included in vivo studies utilizing a combination of the mouse model above. We demonstrated that the deletion of $S m a d 2 / 3$ in adipose tissues has different outcomes in males and females with predominant effects of genotype and diet in females. Smad2/3cKO mice demonstrated a sex-selective effect on weight gain. Smad2/3cKO male mice showed no differences in body weight relative to controls, while female mice gained less weight relative to control littermates on HFD, indicating a sex-influenced role 
for Smad2/3 on adiposity under HFD conditions with less visceral and subcutaneous fat mass relative to controls. Hence female but not male mice are protected from diet induced obesity. This is also consistent with the higher metabolic rates observed in Smad2/3cKO mice and changes in the levels of key lipogenic and lipolytic genes. Considering all data, SMAD2 and SMAD3 not have redundant roles in adipose tissue development or function. SMAD2 and SMAD3 have acquired partial non-redundant functions in adipose tissue development and function as loss of SMAD3 alone protected a subset of mice from DIO in male while loss of both $S m a d 2 / 3$ protected a different subset from DIO corresponding to significant changes in the expression of genes regulating metabolic responses (females only). Considering the differences in the phenotypes observed in Smad3cKO alone (males only) and Smad2/3cKO (females only), we speculate that the different responses of males and females are likely due to the effect of sex-specific hormonal signaling. The combined phenotype is different from either of the single Smad mutants. This study highlights the importance of biological sex as an important modifier of the phenotype observed with the combined deletion of Smad2/3 (Chapter 5).

The third aim of my dissertation included in vitro studies utilizing MEFs isolated from wild type mice and activin A and B deficient double mutant mice. We confirmed the cell autonomous role of activin on preadipocyte proliferation and confirmed that activin A promotes preadipocyte proliferation. We have observed that the combined global knockout of activin A (Inhba) and activin B (Inhbb), enhanced adipocyte differentiation from MEFs, including the appearance of UCP1 positive brown-like cells with increased numbers of mitochondria. Additionally, gene expression profiling, immunofluorescence and western blotting were all consistent with a brown-like cellular phenotype. Collectively, our data indicated that activin signaling may regulate cell proliferation, differentiation, and energy homeostasis (Chapter 6).

Collectively, the results obtained from this study provide additional insight to expand our knowledge about the direct effects of SMAD2/3 on adipocyte development and function and the molecular pathway(s) that regulate adiposity and energy metabolism in response to activin signaling. This study has identified the phenotypic differences between global knockout of Smad3 and adipose-selective knockout of Smad3 (including no report of sexual dimorphism). Also, these findings are informative in determining the importance of activin/SMAD2/3 signaling in the context of obesity and may prove an effective and safe target for therapies aimed at treating or preventing obesity.

\subsection{Future Directions}

This body of work has explored the roles of Smad2, Smad3 and Smad2/3 during mid to-late stages of adipogenesis. Out of these findings, there are several unanswered questions that can be addressed, such as the potential roles of Smad2 and Smad3 in early adipogenesis using an early preadipocyte-specific Cre (Pdgrfa Cre). Additionally, determining if there is redundancy of Smad2 and Smad3 during early adipogenous is highly important given 
the fact that activin (upstream of SMAD2/3) has a clearly established role in this context. It is important to acknowledge the potential limitation of this study with the use the Adiponectin-Cre mouse. With this Cre, early stage adipose-selective gene inactivation occurs in the early to middle to late stages of adipogenesis, starting at the preadipocyte stage and continuing in their descendants. It is plausible that SMAD-mediated events occurring at earlier stages would not be impacted. Thus, to fully understand the role of SMADmediated activin signaling and underlying molecular mechanisms that occur during earlier stages, additional studies are needed using alternative strategies including inducible models that confer spatiotemporal control. This study will further address the activin/SMADs relationship in regulating adipogenesis. The proposed study may also answer if activin regulates preadipocyte proliferation and adipogenesis by phosphorylating SMAD2, SMAD3 or both before adipogenesis begins.

It is very important to investigate the molecular mechanism(s) by which SMAD2/3 modulate sex-selective phenotypes. There are only a few instances, to our knowledge, which show that SMAD3 has a more pronounced effect in males, one for the progression of gonadal tumorigenesis (Q. Li, Graff, et al., 2007), the other, androgen-promoted growth inhibition in the prostate, androgen restricted to males (Hayes et al., 2001). Interestingly, Growth Differentiation Factor 3, another ligand within the TGF- $\beta$ family (that can use the same set of type I receptors as activins), has also shown evidence of sexual dimorphism, with a more pronounced protection from diet-induced obesity in global knockout females (J. J. Shen et al., 2009). Thus, sexual dimorphism within the TGF- $\beta$ family is established. While the mechanisms underlying these phenomena are likely complex, thought to involve both environmental/hormonal and genetic influences (Ober, Loisel, and Gilad, 2008), additional studies are required to better understand these effects in the current model.

Another long-term goal is to enhance our understanding of the developmental origins of "brite adipocy-tes". The future study may include a novel methods to characterize and manipulate primary preadipocytes to study trans-differentiation and to look at the downstream transcription factors including Forkhead box (FOX) that play important roles in cell cycle regulation. The role of FOX is unknown in the context of activin signaling. FOXO1, an inhibitor of proliferation process, activates the promoter of $\mathrm{p} 21$, a negative cell cycle regulator that causes G1 arrest before entering mitotic clonal expansion. SMAD2 and SMAD3 form an activating complex with FOXO1 at the p21 promoter (L. Wang et al., 2018; Seoane et al., 2004). It is plausible that the growth arrest of activin-deficient mice could be associated with the activation of FOXO1. Additionally, FOXC2 participates in white to brown adipocyte conversion by forming a transcriptional complex with SMAD3 and SMAD4 at the plasminiogen activator inhibitor-1 (PAI-1) promoter. This interaction is antagonized by FOXO1 through competition for common DNA binding sites (Fujita et al., 2006). Therefore, investigation of direct roles for FOX/SMADs interactions in transcriptional regulation in context of activin signaling and roles during adipogenesis will likely provide additional mechanistic insight. 
This body of work has discovered an additional insight into the direct effects of Smad2/3 on adipocyte function and the mechanism(s) that regulates adiposity and energy metabolism. The results of this study enhanced our current knowledge and complexity about the adipose-selective effects of Smad2 and Smad3 on adipocyte function and redundancy of both proteins in terms of regulating energy homeostasis and obesity; thereby identifying possible therapeutic targets for the treatment of obesity. Therefore, such studies were essential to investigate the overall impact of SMAD2 and SMAD3 on the progression of obesity and possibly a potential platform to design effective anti-obesity treatments. 


\section{List of References}

Ahima, R. S. and M. A. Lazar (May 2008). "Adipokines and the peripheral and neural control of energy balance". In: Mol Endocrinol 22.5. Edition: 2008/01/19, pp. 1023-31. ISSN: 0888-8809 (Print) 0888-8809. DOI: 10.1210/me.2007-0529.

Allison, D. B. et al. (June 2008). "Obesity as a disease: a white paper on evidence and arguments commissioned by the Council of the Obesity Society". In: Obesity (Silver Spring) 16.6. Edition: 2008/05/10, pp. 1161-77. ISSN: 1930-7381 (Print) 1930-7381. DOI: 10.1038/oby . 2008.231.

Aragon, E. et al. (Nov. 1, 2019). "Structural basis for distinct roles of SMAD2 and SMAD3 in FOXH1 pioneer-directed TGF-beta signaling". In: Genes Dev 33.21. Edition: 2019/10/05, pp. 1506-1524. ISSN: 1549-5477 (Electronic) 0890-9369 (Linking). DOI: 10 . $1101 / \mathrm{gad}$. 330837.119. URL: https://www.ncbi.nlm.nih.gov/pubmed/31582430.

Ashcroft, G. S. et al. (Sept. 1999). "Mice lacking Smad3 show accelerated wound healing and an impaired local inflammatory response". In: Nat Cell Biol 1.5. Edition: 1999/11/13, pp. 260-6. ISSN: 1465-7392 (Print) 1465-7392 (Linking). DOI: 10. 1038/12971. URL: https : //www.ncbi.nlm.nih.gov/pubmed/10559937.

Awan, N. M., I. J. Meurling, and D. O'Shea (Jan. 2021). “Understanding Obesity: The Role of Adipose Tissue Microenvironment and the Gut Microbiome". In: Saudi J Med Med Sci 9.1. Edition: 2021/02/02, pp. 10-15. ISSN: 1658-631X (Print) 2321-4856. DOI: 10.4103/sjmms.sjmms_561_20.

Aykul, Senem et al. (2020). "TGF-B Family Inhibitors Blunt Adipogenesis Via Non-Canonical Regulation Of SMAD Pathways". In: bioRxiv, p. 2020.03.12.988568. DOI: 10.1101/2020 . 03.12.988568. URL: https://www. biorxiv.org/content/biorxiv/early/2020/03/14/ 2020.03.12.988568.full.pdf.

Baglioni, S. et al. (2012). "Functional differences in visceral and subcutaneous fat pads originate from differences in the adipose stem cell". In: PLoS One 7.5. Edition: 2012/05/11, e36569. ISSN: 1932-6203. DOI: 10.1371/journal . pone.0036569.

Bederman, I. R. et al. (Nov. 1, 2018). "Absence of leptin signaling allows fat accretion in cystic fibrosis mice". In: Am J Physiol Gastrointest Liver Physiol 315.5. Edition: 2018/08/18, G685G698. ISSN: 1522-1547 (Electronic) 0193-1857 (Linking). DOI: 10.1152/ajpgi .00344 . 2017. URL: https://www.ncbi.nlm.nih.gov/pubmed/30118352.

Bernard, D. J., K. B. Lee, and M. M. Santos (Oct. 13, 2006). "Activin B can signal through both ALK4 and ALK7 in gonadotrope cells". In: Reprod Biol Endocrinol 4. Edition: 2006/10/17, p. 52. ISSN: 1477-7827. DOI: $10.1186 / 1477-7827-4-52$. 
Bernhard, F. et al. (Feb. 1, 2013). "Functional relevance of genes implicated by obesity genome-wide association study signals for human adipocyte biology". In: Diabetologia 56.2, pp. 311-322. ISSN: 1432-0428. DOI: 10 . 1007 / s00125-012-2773-0. URL: https : //doi.org/10.1007/s00125-012-2773-0.

Bonomi, L. et al. (Sept. 1, 2012). "Activin B regulates islet composition and islet mass but not whole body glucose homeostasis or insulin sensitivity". In: Am J Physiol Endocrinol Metab 303.5. Edition: 2012/06/29, E587-96. ISSN: 1522-1555 (Electronic) 0193-1849 (Linking). DOI: 10 . 1152/ajpendo .00177 . 2012. URL: https : / www . ncbi . nlm . nih . gov/pubmed/ 22739106.

Bournat, J. C. and C. W. Brown (Oct. 2010). "Mitochondrial dysfunction in obesity". In: Curr Opin Endocrinol Diabetes Obes 17.5. Edition: 2010/06/30, pp. 446-52. ISSN: 1752-2978 (Electronic) 1752-296X (Linking). DOI: 10 . 1097 / MED . 0b013e32833c3026. URL: https : //www.ncbi.nlm.nih.gov/pubmed/20585248.

Boutens, L. and R. Stienstra (May 2016). "Adipose tissue macrophages: going off track during obesity". In: Diabetologia 59.5. Edition: 2016/03/05, pp. 879-94. ISSN: 0012-186X (Print) 0012-186x. DOI: 10.1007/s00125-016-3904-9.

Bowers, R. R. et al. (Aug. 29, 2006). "Stable stem cell commitment to the adipocyte lineage by inhibition of DNA methylation: role of the BMP-4 gene". In: Proc Natl Acad Sci U $S$ A 103.35. Edition: 2006/08/19, pp. 13022-7. ISSN: 0027-8424 (Print) 0027-8424. DOI: 10.1073/pnas.0605789103.

Brown, C. W., D. E. Houston-Hawkins, et al. (Aug. 2000). "Insertion of Inhbb into the Inhba locus rescues the Inhba-null phenotype and reveals new activin functions". In: Nat Genet 25.4. Edition: 2000/08/10, pp. 453-7. ISSN: 1061-4036 (Print) 1061-4036 (Linking). DOI: 10.1038/78161. URL: https://www.ncbi.nlm.nih.gov/pubmed/10932194.

Brown, C. W., L. Li, et al. (Dec. 2003). "Activins are critical modulators of growth and survival". In: Mol Endocrinol 17.12. Edition: 2003/10/11, pp. 2404-17. ISSN: 0888-8809 (Print) 0888-8809 (Linking). DOI: 10.1210/me.2003-0051. URL: https : //www . ncbi .nlm . nih.gov/pubmed/14551263.

Brown, K. A., J. A. Pietenpol, and H. L. Moses (May 1, 2007). "A tale of two proteins: differential roles and regulation of Smad2 and Smad3 in TGF-beta signaling". In: J Cell Biochem 101.1. Edition: 2007/03/07, pp. 9-33. ISSN: 0730-2312 (Print) 0730-2312. DOI: 10.1002/jcb. 21255.

Butler, Merlin G. (2016). "Single Gene and Syndromic Causes of Obesity: Illustrative Examples". In: Prog Mol Biol Transl Sci 140. Edition: 2016/03/23, pp. 1-45. ISSN: 18780814 1877-1173. DOI: 10 . 1016/bs . pmbts . 2015 . 12 .003. PMID: 27288824. URL: https : //pubmed.ncbi.nlm.nih.gov/27288824\%20https : / /www . ncbi.nlm.nih.gov/pmc/ articles/PMC7377403/.

Cannon, B. and J. Nedergaard (Jan. 2004). "Brown adipose tissue: function and physiological significance". In: Physiol Rev 84.1. Edition: 2004/01/13, pp. 277-359. ISSN: 0031-9333 (Print) 0031-9333. DOI: 10.1152/physrev.00015.2003.

Cao, L. et al. (Sept. 7, 2011). "White to brown fat phenotypic switch induced by genetic and environmental activation of a hypothalamic-adipocyte axis". In: Cell Metab 14.3. Edition: 
2011/09/13, pp. 324-38. ISSN: 1550-4131 (Print) 1550-4131. DOI: 10 . 1016/j . cmet. 2011. 06.020.

Cao, W. et al. (Apr. 2004). "p38 mitogen-activated protein kinase is the central regulator of cyclic AMP-dependent transcription of the brown fat uncoupling protein 1 gene". In: $\mathrm{Mol}$ Cell Biol 24.7. Edition: 2004/03/17, pp. 3057-67. ISSN: 0270-7306 (Print) 0270-7306. DOI: $10.1128 / \mathrm{mcb} \cdot 24.7 .3057-3067.2004$.

Carlsson, Lena M. S. et al. (May 1, 2009). "ALK7 expression is specific for adipose tissue, reduced in obesity and correlates to factors implicated in metabolic disease". In: Biochemical and Biophysical Research Communications 382.2, pp. 309-314. ISSN: 0006-291X. DOI: 10.1016/ j . bbrc . 2009 .03 .014. URL: https : / / www . sciencedirect . com/science / article/pii/S0006291X09004665.

Carmody, R. N. et al. (Jan. 14, 2015). "Diet dominates host genotype in shaping the murine gut microbiota". In: Cell Host Microbe 17.1. Edition: 2014/12/24, pp. 72-84. ISSN: 1931-3128 (Print) 1931-3128. DOI: 10.1016/j . chom.2014.11.010.

Cawthorn, W. P. et al. (Feb. 2012). "Wnt6, Wnt10a and Wnt10b inhibit adipogenesis and stimulate osteoblastogenesis through a $\beta$-catenin-dependent mechanism". In: Bone 50.2. Edition: 2011/08/30, pp. 477-89. ISSN: 8756-3282 (Print) 1873-2763. DOI: $10.1016 / \mathrm{j}$. bone . 2011.08 .010$.

Chang, H., C. W. Brown, and M. M. Matzuk (Dec. 2002). "Genetic analysis of the mammalian transforming growth factor-beta superfamily". In: Endocr Rev 23.6. Edition: 2002/12/06, pp. 787-823. ISSN: 0163-769X (Print) 0163-769x. DOI: 10.1210/er . 2002-0003.

Cheng, M. et al. (2018). "Computational analyses of obesity associated loci generated by genome-wide association studies". In: PLoS One 13.7. Edition: 2018/07/03, e0199987. ISSN: 1932-6203. DOI: 10.1371/journal . pone. 0199987.

Chevalier, Claire et al. (Dec. 3, 2015). “Gut Microbiota Orchestrates Energy Homeostasis during Cold". In: Cell 163.6, pp. 1360-1374. ISSN: 0092-8674. DOI: 10 . 1016 / j . cell . 2015 . 11 . 004. URL: https : / / www . sciencedirect . com / science / article / pii / S0092867415014841.

Choe, S. S. et al. (2016). "Adipose Tissue Remodeling: Its Role in Energy Metabolism and Metabolic Disorders". In: Front Endocrinol (Lausanne) 7. Edition: 2016/05/06, p. 30. ISSN: 1664-2392 (Print) 1664-2392 (Linking). DOI: 10 . 3389 / fendo . 2016 . 00030. URL: https://www.ncbi.nlm.nih.gov/pubmed/27148161.

Chooi, Y. C., C. Ding, and F. Magkos (Mar. 2019). "The epidemiology of obesity". In: Metabolism 92. Edition: 2018/09/27, pp. 6-10. ISSN: 1532-8600 (Electronic) 0026-0495 (Linking). DOI: 10.1016/j . metabol . 2018.09.005. URL: https ://www . ncbi . nlm.nih . gov/pubmed/30253139.

Choy, L., J. Skillington, and R. Derynck (May 1, 2000). "Roles of autocrine TGF-beta receptor and Smad signaling in adipocyte differentiation". In: J Cell Biol 149.3. Edition: 2000/05/03, pp. 667-82. ISSN: 0021-9525 (Print) 0021-9525. DOI: 10.1083/jcb.149.3.667.

Coe, N. R., M. A. Simpson, and D. A. Bernlohr (May 1999). "Targeted disruption of the adipocyte lipid-binding protein (aP2 protein) gene impairs fat cell lipolysis and increases 
cellular fatty acid levels". In: J Lipid Res 40.5. Edition: 1999/05/01, pp. 967-72. ISSN: 0022-2275 (Print) 0022-2275.

Dani, C. (Feb. 2013). "Activins in adipogenesis and obesity". In: International journal of obesity (2005) 37.2. Edition: 2012/03/01, pp. 163-6. ISSN: 0307-0565. DOI: 10.1038/i jo. 2012.28. Daniel, S. G. et al. (Sept. 2017). "Functional Changes in the Gut Microbiome Contribute to Transforming Growth Factor $\beta$-Deficient Colon Cancer". In: mSystems 2.5. Edition: 2017/09/28. ISSN: 2379-5077 (Print) 2379-5077. DOI: 10.1128/mSystems . 00065- 17.

Darlington, G. J., S. E. Ross, and O. A. MacDougald (Nov. 13, 1998). “The role of C/EBP genes in adipocyte differentiation". In: J Biol Chem 273.46. Edition: 1998/11/07, pp. 3005760. ISSN: 0021-9258 (Print) 0021-9258 (Linking). DOI: 10 . 1074/ jbc . 273 . 46 . 30057. URL: https://www.ncbi.nlm.nih.gov/pubmed/9804754.

Darrah, R. J. et al. (July 2013). “Ventilatory pattern and energy expenditure are altered in cystic fibrosis mice". In: J Cyst Fibros 12.4. Edition: 2013/01/08, pp. 345-51. ISSN: 1873-5010 (Electronic) 1569-1993 (Linking). DOI: 10 . 1016/ j . jcf . 2012 . 11 . 008. URL: https://www.ncbi.nlm.nih.gov/pubmed/23290341.

Datto, M. B. et al. (Apr. 1999). "Targeted disruption of Smad3 reveals an essential role in transforming growth factor beta-mediated signal transduction". In: Mol Cell Biol 19.4. Edition: 1999/03/19, pp. 2495-504. ISSN: 0270-7306 (Print) 0270-7306 (Linking). DOI: 10.1128/mcb.19.4.2495. URL: https://www.ncbi.nlm.nih.gov/pubmed/10082515.

DeFronzo, R. A. (July 2010). "Insulin resistance, lipotoxicity, type 2 diabetes and atherosclerosis: the missing links. The Claude Bernard Lecture 2009". In: Diabetologia 53.7. Edition: 2010/04/03, pp. 1270-87. ISSN: 0012-186X (Print) 0012-186x. DOI: 10 .1007/s00125-010$1684-1$.

Derynck, R. and E. H. Budi (Feb. 26, 2019). "Specificity, versatility, and control of TGF$\beta$ family signaling". In: Sci Signal 12.570. Edition: 2019/02/28. ISSN: 1945-0877 (Print) 1945-0877. DOI: 10.1126/scisignal . aav5183.

Dijke, P. ten and C. S. Hill (May 2004). "New insights into TGF-beta-Smad signalling". In: Trends Biochem Sci 29.5. Edition: 2004/05/08, pp. 265-73. ISSN: 0968-0004 (Print) 0968-0004. DOI: $10.1016 /$ j.tibs. 2004.03.008.

Duca, F. A. et al. (May 2014). "Replication of obesity and associated signaling pathways through transfer of microbiota from obese-prone rats". In: Diabetes 63.5. Edition: 2014/01/17, pp. 1624-36. ISSN: 0012-1797. DOI: 10.2337/db13-1526.

Duncan, Robin E. et al. (2007). "Regulation of lipolysis in adipocytes". In: Annu Rev Nutr 27, pp. 79-101. ISSN: 0199-9885 1545-4312. DOI: 10.1146/annurev . nutr. 27.061406. 093734. PMID: 17313320. URL: https://pubmed.ncbi.nlm.nih.gov/17313320\%20https://www . ncbi.nlm.nih.gov/pmc/articles/PMC2885771/.

Dunn, N. R. et al. (Apr. 2004). “Combinatorial activities of Smad2 and Smad3 regulate mesoderm formation and patterning in the mouse embryo". In: Development 131.8. Edition: 2004/04/16, pp. 1717-28. ISSN: 0950-1991 (Print) 0950-1991 (Linking). DOI: 10 . 1242/dev . 01072. URL: https://www.ncbi.nlm.nih.gov/pubmed/15084457.

Eguchi, J. et al. (Mar. 2, 2011). "Transcriptional control of adipose lipid handling by IRF4". In: Cell Metab 13.3. Edition: 2011/03/02, pp. 249-59. ISSN: 1932-7420 (Electronic) 1550-4131 
(Linking). DOI: 10.1016/j.cmet.2011.02.005. URL: https://www.ncbi.nlm.nih.gov/ pubmed/21356515.

Engel, M. E. et al. (Dec. 24, 1999). "Interdependent SMAD and JNK signaling in transforming growth factor-beta-mediated transcription". In: J Biol Chem 274.52. Edition: 1999/12/22, pp. 37413-20. ISSN: 0021-9258 (Print) 0021-9258. DOI: 10.1074/ jbc. 274.52 . 37413.

Fajas, L. (2003). “Adipogenesis: a cross-talk between cell proliferation and cell differentiation". In: Ann Med 35.2. Edition: 2003/06/11, pp. 79-85. ISSN: 0785-3890 (Print) 0785-3890. DOI: $10.1080 / 07853890310009999$.

Farmer, Stephen R. (2006). "Transcriptional control of adipocyte formation". In: Cell Metab 4.4, pp. 263-273. ISSN: 1550-4131. DOI: 10.1016/j . cmet.2006.07.001. PMID: 17011499. URL: https : / pubmed.ncbi.nlm.nih.gov/17011499\%20https : //www . ncbi.nlm.nih . gov/pmc/articles/PMC1958996/.

Fei, T. et al. (Dec. 2010). "Smad2 mediates Activin/Nodal signaling in mesendoderm differentiation of mouse embryonic stem cells". In: Cell Res 20.12. Edition: 2010/11/17, pp. 1306-18. ISSN: 1748-7838 (Electronic) 1001-0602 (Linking). DOI: 10 .1038/cr . 2010 . 158. URL: https://www.ncbi.nlm.nih.gov/pubmed/21079647.

Feng, X. H. and R. Derynck (2005). "Specificity and versatility in tgf-beta signaling through Smads". In: Annu Rev Cell Dev Biol 21. Edition: 2005/10/11, pp. 659-93. ISSN: 1081-0706 (Print) 1081-0706. DOI: 10.1146/annurev . cellbio.21.022404.142018.

Fleming, N. I. et al. (Jan. 15, 2013). "SMAD2, SMAD3 and SMAD4 mutations in colorectal cancer". In: Cancer Res 73.2. Edition: 2012/11/10, pp. 725-35. ISSN: 1538-7445 (Electronic) 0008-5472 (Linking). DOI: 10 . 1158/0008-5472. CAN-12-2706. URL: https : //www . ncbi . nlm.nih.gov/pubmed/23139211.

Fontana, L. et al. (Apr. 2007). "Visceral fat adipokine secretion is associated with systemic inflammation in obese humans". In: Diabetes 56.4. Edition: 2007/02/09, pp. 1010-3. ISSN: 0012-1797 (Print) 0012-1797. DOI: 10.2337/db06-1656.

Fortier, M. et al. (Aug. 2004). "Hormone-sensitive lipase-independent adipocyte lipolysis during beta-adrenergic stimulation, fasting, and dietary fat loading". In: Am J Physiol Endocrinol Metab 287.2. Edition: 2004/07/24, E282-8. ISSN: 0193-1849 (Print) 0193-1849. DOI: 10.1152/ajpendo.00203.2003.

Frayn, K. N. (Mar. 2000). "Visceral fat and insulin resistance-causative or correlative?" In: Br J Nutr 83 Suppl 1. Edition: 2000/07/13, S71-7. ISSN: 0007-1145 (Print) 0007-1145. DOI: $10.1017 / \mathrm{s} 0007114500000982$.

Frühbeck, Gema et al. (2014). "Regulation of adipocyte lipolysis". In: Nutrition Research Reviews 27.1. Edition: 2014/05/28, pp. 63-93. ISSN: 0954-4224. DOI: 10 . 1017 / S095442241400002X. URL: https : / /www . cambridge . org/core/article/regulationof-adipocyte-lipolysis/08D91A549DE1C469D6265EDB5985B95A.

Fu, Y. et al. (Oct. 17, 2003). “The p38 MAPK inhibitor, PD169316, inhibits transforming growth factor beta-induced Smad signaling in human ovarian cancer cells". In: Biochem Biophys Res Commun 310.2. Edition: 2003/10/03, pp. 391-7. ISSN: 0006-291X (Print) 0006291x. DOI: $10.1016 / \mathrm{j}$.bbrc.2003.09.021. 
Fujita, H. et al. (Mar. 17, 2006). "Foxc2 is a common mediator of insulin and transforming growth factor beta signaling to regulate plasminogen activator inhibitor type I gene expression". In: Circ Res 98.5. Edition: 2006/02/04, pp. 626-34. ISSN: 0009-7330. DOI: 10.1161/01.RES.0000207407.51752.3c.

Gao, C. L. et al. (May 14, 2010). "Mitochondrial dysfunction is induced by high levels of glucose and free fatty acids in 3T3-L1 adipocytes". In: Mol Cell Endocrinol 320.1. Edition: 2010/02/11, pp. 25-33. ISSN: 0303-7207. DOI: 10.1016/j .mce.2010.01.039.

Gardan, D., F. Gondret, and I. Louveau (Aug. 2006). "Lipid metabolism and secretory function of porcine intramuscular adipocytes compared with subcutaneous and perirenal adipocytes". In: Am J Physiol Endocrinol Metab 291.2. Edition: 2006/05/18, E372-80. ISSN: 0193-1849 (Print) 0193-1849 (Linking). DOI: 10.1152/ajpendo.00482 . 2005. URL: https : //www.ncbi.nlm.nih.gov/pubmed/16705057.

Gesta, S., Y. H. Tseng, and C. R. Kahn (Oct. 19, 2007). “Developmental origin of fat: tracking obesity to its source". In: Cell 131.2. Edition: 2007/10/25, pp. 242-56. ISSN: 0092-8674 (Print) 0092-8674. DOI: 10.1016/j . cell.2007.10.004.

Giovannucci, Edward et al. (2010). "Diabetes and cancer: a consensus report". In: Diabetes Care 33.7, pp. 1674-1685. ISSN: 1935-5548 0149-5992. DOI: 10 . 2337 /dc10 - 0666. PMID: 20587728. URL: https : //pubmed.ncbi.nlm.nih.gov/20587728\%20https : //www . ncbi . nlm.nih.gov/pmc/articles/PMC2890380/.

Giralt, Marta and Francesc Villarroya (2013). "White, Brown, Beige/Brite: Different Adipose Cells for Different Functions?" In: Endocrinology 154.9, pp. 2992-3000. ISSN: 0013-7227. DOI: 10 .1210/en. 2013-1403. URL: https : //doi .org/10 .1210/en . 2013-1403 (visited on $07 / 04 / 2021)$.

Golozoubova, V., B. Cannon, and J. Nedergaard (Aug. 2006). “UCP1 is essential for adaptive adrenergic nonshivering thermogenesis". In: Am J Physiol Endocrinol Metab 291.2. Edition: 2006/04/06, E350-7. ISSN: 0193-1849 (Print) 0193-1849. DOI: 10 . 1152/ajpendo. 00387. 2005.

Golozoubova, V., E. Hohtola, et al. (Sept. 2001). “Only UCP1 can mediate adaptive nonshivering thermogenesis in the cold". In: Faseb j 15.11. Edition: 2001/08/21, pp. 2048-50. ISSN: 0892-6638 (Print) 0892-6638. DOI: 10.1096/fj .00-0536f je.

Grundy, Scott M. (2004). "Obesity, Metabolic Syndrome, and Cardiovascular Disease”. In: The Journal of Clinical Endocrinology \& Metabolism 89.6, pp. 2595-2600. ISSN: 0021-972X. DOI: $10.1210 /$ jc. 2004-0372. URL: https : //doi .org/10.1210/jc. $2004-0372$ (visited on $06 / 19 / 2021)$.

Guerra, C. et al. (1998). "Emergence of brown adipocytes in white fat in mice is under genetic control. Effects on body weight and adiposity". In: J Clin Invest 102.2, pp. 412-420. ISSN: 0021-9738. DOI: 10 . 1172/JCI3155. PMID: 9664083. URL: https : / pubmed . ncbi . nlm.nih.gov/9664083\%20https://www.ncbi.nlm.nih.gov/pmc/articles/PMC508900/.

Haemmerle, G. et al. (Feb. 15, 2002). "Hormone-sensitive lipase deficiency in mice causes diglyceride accumulation in adipose tissue, muscle, and testis". In: J Biol Chem 277.7. Edition: 2001/11/22, pp. 4806-15. ISSN: 0021-9258 (Print) 0021-9258. DOI: 10.1074/jbc . M110355200. 
Hajer, G. R., T. W. van Haeften, and F. L. Visseren (Dec. 2008). “Adipose tissue dysfunction in obesity, diabetes, and vascular diseases". In: Eur Heart J 29.24. Edition: 2008/09/09, pp. 2959-71. ISSN: 0195-668x. DOI: 10.1093/eurheartj/ehn387.

Hanafusa, H. et al. (Sept. 17, 1999). "Involvement of the p38 mitogen-activated protein kinase pathway in transforming growth factor-beta-induced gene expression". In: J Biol Chem 274.38. Edition: 1999/09/10, pp. 27161-7. ISSN: 0021-9258 (Print) 0021-9258. DOI: $10.1074 / \mathrm{jbc} .274 .38 .27161$.

Hayes, S. A. et al. (Mar. 1, 2001). "SMAD3 represses androgen receptor-mediated transcription". In: Cancer Res 61.5. Edition: 2001/03/31, pp. 2112-8. ISSN: 0008-5472 (Print) 0008-5472.

Herrera, Blanca M. and Cecilia M. Lindgren (2010). "The genetics of obesity". In: Curr Diab Rep 10.6, pp. 498-505. ISSN: 1539-0829 1534-4827. DOI: 10.1007/s11892-010-0153z. PMID: 20931363. URL: https : / / pubmed . ncbi .nlm . nih . gov/20931363\%20https : //www.ncbi.nlm.nih.gov/pmc/articles/PMC2955913/.

Heyer, J. et al. (Oct. 26, 1999). "Postgastrulation Smad2-deficient embryos show defects in embryo turning and anterior morphogenesis". In: Proc Natl Acad Sci U S A 96.22. Edition: 1999/10/27, pp. 12595-600. ISSN: 0027-8424 (Print) 0027-8424 (Linking). DOI: 10.1073/pnas.96.22.12595. URL: https://www.ncbi.nlm.nih.gov/pubmed/10535967.

Hildebrandt, Marie A. et al. (Nov. 1, 2009). "High-Fat Diet Determines the Composition of the Murine Gut Microbiome Independently of Obesity". In: Gastroenterology 137.5, 1716-1724.e2. ISSN: 0016-5085. DOI: 10 . 1053 / j . gastro . 2009 . 08 . 042. URL: https : //www.sciencedirect.com/science/article/pii/s0016508509014577.

Hill, Caroline S. (2016). "Transcriptional Control by the SMADs". In: Cold Spring Harb Perspect Biol 8.10, a022079. ISSN: 1943-0264. DOI: 10.1101/cshperspect . a022079. PMID: 27449814. URL: https : //pubmed.ncbi.nlm.nih.gov/27449814\%20https : //www .ncbi . nlm.nih.gov/pmc/articles/PMC5046698/.

Hirai, S. et al. (Mar. 31, 2005). "Activin A inhibits differentiation of 3T3-L1 preadipocyte". In: Mol Cell Endocrinol 232.1. Edition: 2005/03/02, pp. 21-6. ISSN: 0303-7207 (Print) 0303-7207 (Linking). DOI: 10.1016/j.mce.2005.01.001. URL: https ://www .ncbi.nlm.nih.gov/ pubmed/15737465.

Holm, C. (Dec. 2003). "Molecular mechanisms regulating hormone-sensitive lipase and lipolysis". In: Biochem Soc Trans 31 (Pt 6). Edition: 2003/12/04, pp. 1120-4. ISSN: 0300-5127 (Print) 0300-5127. DOI: 10.1042/bst0311120.

Horie, T. et al. (June 2008). "TG-interacting factor is required for the differentiation of preadipocytes". In: J Lipid Res 49.6. Edition: 2008/03/04, pp. 1224-34. ISSN: 0022-2275 (Print) 0022-2275. DOI: 10.1194/jlr . M700578-JLR200.

Hruby, Adela and Frank B. Hu (2015). "The Epidemiology of Obesity: A Big Picture". In: Pharmacoeconomics 33.7, pp. 673-689. ISSN: 1179-2027 1170-7690. DOI: 10.1007/s40273014-0243-x. PMID: 25471927. URL: https : / / pubmed .ncbi .nlm.nih.gov/25471927\% 20https://www.ncbi.nlm.nih.gov/pmc/articles/PMC4859313/. 
$\mathrm{Hu}$, J. et al. (Oct. 15, 2016). "Activin A stimulates the proliferation and differentiation of cardiac fibroblasts via the ERK1/2 and p38-MAPK pathways". In: Eur J Pharmacol 789. Edition: 2016/08/02, pp. 319-327. ISSN: 0014-2999. DOI: 10.1016/j . ejphar . 2016 .07 . 053.

Huang, E. Y. et al. (Nov. 2013). "Composition of dietary fat source shapes gut microbiota architecture and alters host inflammatory mediators in mouse adipose tissue". In: JPEN J Parenter Enteral Nutr 37.6. Edition: 2013/05/04, pp. 746-54. ISSN: 0148-6071 (Print) 0148-6071. DOI: 10.1177/0148607113486931.

Huang, Qingyang (Mar. 20, 2015). "Genetic Study of Complex Diseases in the Post-GWAS Era". In: Journal of Genetics and Genomics 42.3, pp. 87-98. ISSN: 1673-8527. DOI: 10.1016/ j.jgg. 2015.02.001. URL: https : //www. sciencedirect.com/science/article/pii/ S1673852715000326.

Huang, S., B. Chen, C. Humeres, et al. (July 2020). "The role of Smad2 and Smad3 in regulating homeostatic functions of fibroblasts in vitro and in adult mice". In: Biochim Biophys Acta Mol Cell Res 1867.7. Edition: 2020/03/18, p. 118703. ISSN: 0167-4889 (Print) 0167-4889. DOI: 10.1016/j . bbamcr. 2020.118703.

Huang, S., B. Chen, Y. Su, et al. (July 2019). “Distinct roles of myofibroblast-specific Smad2 and Smad3 signaling in repair and remodeling of the infarcted heart". In: J Mol Cell Cardiol 132. Edition: 2019/05/16, pp. 84-97. ISSN: 0022-2828 (Print) 0022-2828. DOI: $10.1016 / \mathrm{j}$. yjmcc. 2019.05.006.

Huang, Xingjun et al. (2018). "The PI3K/AKT pathway in obesity and type 2 diabetes". In: Int J Biol Sci 14.11, pp. 1483-1496. ISSN: 1449-2288. DOI: 10.7150/ijbs.27173. PMID: 30263000. URL: https://pubmed.ncbi.nlm.nih.gov/30263000\%20https : / www . ncbi . nlm.nih.gov/pmc/articles/PMC6158718/.

Hüls, Anke et al. (June 1, 2021). "Polygenic risk for obesity and its interaction with lifestyle and sociodemographic factors in European children and adolescents". In: International Journal of Obesity 45.6, pp. 1321-1330. ISSN: 1476-5497. DOI: 10.1038/s41366-021-007955. URL: https://doi .org/10.1038/s41366-021-00795-5.

Ibrahim, M. M. (Jan. 2010). "Subcutaneous and visceral adipose tissue: structural and functional differences". In: Obes Rev 11.1. Edition: 2009/08/07, pp. 11-8. ISSN: 1467-7881. DOI: $10.1111 / j .1467-789 x .2009 .00623$.x.

Itman, C. et al. (July 2009). "Developmentally regulated SMAD2 and SMAD3 utilization directs activin signaling outcomes". In: Dev Dyn 238.7. Edition: 2009/06/12, pp. 1688-700. ISSN: 1058-8388 (Print) 1058-8388 (Linking). DOI: 10 . 1002 / dvdy . 21995. URL: https : //www.ncbi.nlm.nih.gov/pubmed/19517569.

Jinnin, M., H. Ihn, and K. Tamaki (Feb. 2006). "Characterization of SIS3, a novel specific inhibitor of Smad3, and its effect on transforming growth factor-beta1-induced extracellular matrix expression". In: Mol Pharmacol 69.2. Edition: 2005/11/17, pp. 597-607. ISSN: 0026-895X (Print) 0026-895X (Linking). DOI: 10.1124/mol .105. 017483. URL: https : //www.ncbi.nlm.nih.gov/pubmed/16288083.

Jo, J. et al. (Mar. 2009). "Hypertrophy and/or Hyperplasia: Dynamics of Adipose Tissue Growth". In: PLoS Comput Biol 5.3. Edition: 2009/03/28, e1000324. ISSN: 1553-7358 
(Electronic) 1553-734X (Linking). DOI: 10 . 1371 / journal . pcbi . 1000324. URL: https : //www.ncbi.nlm.nih.gov/pubmed/19325873.

$\mathrm{Ju}$, W. et al. (Jan. 2006). "Deletion of Smad2 in mouse liver reveals novel functions in hepatocyte growth and differentiation". In: Mol Cell Biol 26.2. Edition: 2005/12/31, pp. 654-67. ISSN: 0270-7306 (Print) 0270-7306 (Linking). DOI: 10 .1128/MCB . 26 . 2 .654-667 . 2006. URL: https://www.ncbi.nlm.nih.gov/pubmed/16382155.

Karagozian, Raffi, Zoltán Derdák, and György Baffy (May 1, 2014). “Obesity-associated mechanisms of hepatocarcinogenesis". In: Metabolism 63.5, pp. 607-617. ISSN: 0026-0495. DOI: 10 . 1016/ j . metabol . 2014 .01 .011. URL: https : / / www . sciencedirect . com / science/article/pii/S0026049514000365.

Kashiwagi, I. et al. (July 21, 2015). "Smad2 and Smad3 Inversely Regulate TGF-beta Autoinduction in Clostridium butyricum-Activated Dendritic Cells". In: Immunity 43.1. Edition: 2015/07/05, pp. 65-79. ISSN: 1097-4180 (Electronic) 1074-7613 (Linking). DOI: 10.1016/j . immuni.2015.06.010. URL: https://www.ncbi.nlm.nih.gov/pubmed/26141582.

Khera, A. V. et al. (Apr. 18, 2019). "Polygenic Prediction of Weight and Obesity Trajectories from Birth to Adulthood". In: Cell 177.3. Edition: 2019/04/20, 587-596.e9. ISSN: 0092-8674 (Print) 0092-8674. DOI: 10.1016/j.cell.2019.03.028.

Kim, Jaeseob et al. (July 1, 1997). "Drosophila Mad binds to DNA and directly mediates activation of vestigial by Decapentaplegic". In: Nature 388.6639, pp. 304-308. ISSN: 14764687. DOI: 10.1038/40906. URL: https://doi .org/10.1038/40906.

Konrad, D., A. Rudich, and E. J. Schoenle (Apr. 1, 2007). "Improved glucose tolerance in mice receiving intraperitoneal transplantation of normal fat tissue". In: Diabetologia 50.4, pp. 833-839. ISSN: 1432-0428. DOI: 10 . 1007 / s00125-007-0596-1. URL: https : //doi.org/10.1007/s00125-007-0596-1.

Kriseman, M. et al. (Feb. 26, 2019). "Uterine double-conditional inactivation of Smad2 and Smad3 in mice causes endometrial dysregulation, infertility, and uterine cancer". In: Proc Natl Acad Sci U S A 116.9. Edition: 2019/01/18, pp. 3873-3882. ISSN: 1091-6490 (Electronic) 0027-8424 (Linking). DOI: 10.1073/pnas .1806862116. URL: https ://www . ncbi.nlm.nih. gov/pubmed/30651315.

Kroon, L. M. de et al. (Feb. 27, 2017). "SMAD3 and SMAD4 have a more dominant role than SMAD2 in TGF $\beta$-induced chondrogenic differentiation of bone marrow-derived mesenchymal stem cells". In: Sci Rep 7. Edition: 2017/02/28, p. 43164. ISSN: 2045-2322. DOI: $10.1038 /$ srep43164.

Kumari, Roshan et al. (Nov. 2021). "SMAD2 and SMAD3 differentially regulate adiposity and the growth of subcutaneous white adipose tissue". In: FASEB journal: official publication of the Federation of American Societies for Experimental Biology. In press.

Kurokawa, M. et al. (July 2, 1998). "The oncoprotein Evi-1 represses TGF-beta signalling by inhibiting Smad3". In: Nature 394.6688. Edition: 1998/07/17, pp. 92-6. ISSN: 0028-0836 (Print) 0028-0836. DOI: $10.1038 / 27945$.

Latres, Esther et al. (Apr. 28, 2017). "Activin A more prominently regulates muscle mass in primates than does GDF8". In: Nature Communications 8.1, p. 15153. ISSN: 2041-1723. DOI: 10.1038/ncomms15153. URL: https://doi.org/10.1038/ncomms15153. 
Lee, Bonggi and Jianhua Shao (Aug. 1, 2012). "Adiponectin and lipid metabolism in skeletal muscle". In: Acta Pharmaceutica Sinica B 2.4, pp. 335-340. ISSN: 2211-3835. DOI: 10.1016/ j . apsb. 2012.06 .008. URL: https ://www . sciencedirect.com/science/article/pii/ S2211383512000925.

Lee, J. H. et al. (Mar. 13, 2020). "Protection from beta-cell apoptosis by inhibition of TGFbeta/Smad3 signaling". In: Cell Death Dis 11.3. Edition: 2020/03/15, p. 184. ISSN: 20414889 (Electronic). DOI: 10.1038/s41419-020-2365-8. URL: https://www .ncbi.nlm.nih. gov/pubmed/32170115.

Lee, M. J. (Apr. 2018). "Transforming growth factor beta superfamily regulation of adipose tissue biology in obesity". In: Biochim Biophys Acta Mol Basis Dis 1864.4. Edition: 2018/02/08, pp. 1160-1171. ISSN: 0925-4439 (Print) 0925-4439 (Linking). DOI: 10 .1016/j . bbadis. 2018.01.025. URL: https://www.ncbi.nlm.nih.gov/pubmed/29409985.

Lefevre, C. et al. (2019). "Metabolic Phenotyping of Adipose-Derived Stem Cells Reveals a Unique Signature and Intrinsic Differences between Fat Pads". In: Stem Cells Int 2019. Edition: 2019/06/22, p. 9323864. ISSN: 1687-966X (Print). DOI: 10.1155/2019/9323864.

Lehner, R. and A. Kuksis (1996). "Biosynthesis of triacylglycerols". In: Prog Lipid Res 35.2. Edition: 1996/01/01, pp. 169-201. ISSN: 0163-7827 (Print) 0163-7827. DOI: 10.1016/01637827 (96) 00005-7.

Li, B. et al. (Mar. 5, 2019). "Microbiota Depletion Impairs Thermogenesis of Brown Adipose Tissue and Browning of White Adipose Tissue". In: Cell Rep 26.10. Edition: 2019/03/07, 2720-2737.e5. DOI: 10.1016/j . celrep. 2019.02.015.

Li, L. et al. (Aug. 2009). "Activin signaling: effects on body composition and mitochondrial energy metabolism”. In: Endocrinology 150.8. Edition: 2009/04/25, pp. 3521-9. ISSN: 19457170 (Electronic) 0013-7227 (Linking). DOI: 10 . 1210/en . 2008-0922. URL: https : //www . ncbi.nlm.nih.gov/pubmed/19389832.

Li, Q., J. M. Graff, et al. (Oct. 2007). "SMAD3 regulates gonadal tumorigenesis". In: Mol Endocrinol 21.10. Edition: 2007/06/28, pp. 2472-86. ISSN: 0888-8809 (Print) 0888-8809. DOI: 10.1210/me.2007-0147.

Li, Q., S. A. Pangas, et al. (Dec. 2008). "Redundant roles of SMAD2 and SMAD3 in ovarian granulosa cells in vivo". In: Mol Cell Biol 28.23. Edition: 2008/09/24, pp. 7001-11. ISSN: 1098-5549 (Electronic) 0270-7306 (Linking). DOI: 10 . 1128/MCB . 00732 - 08. URL: https : //www.ncbi.nlm.nih.gov/pubmed/18809571.

Lin, H. M. et al. (May 1, 2009). "Transforming growth factor-beta/Smad3 signaling regulates insulin gene transcription and pancreatic islet beta-cell function". In: J Biol Chem 284.18. Edition: 2009/03/07, pp. 12246-57. ISSN: 0021-9258 (Print) 0021-9258. DOI: 10.1074/jbc . M805379200.

Lin, Y. et al. (Feb. 11, 2005). "The hyperglycemia-induced inflammatory response in adipocytes: the role of reactive oxygen species". In: J Biol Chem 280.6. Edition: 2004/11/13, pp. 4617-26. ISSN: 0021-9258 (Print) 0021-9258. DOI: $10.1074 / \mathrm{jbc}$. M411863200.

Liu, L. et al. (Feb. 24, 2016). "Smad2 and Smad3 have differential sensitivity in relaying TGFbeta signaling and inversely regulate early lineage specification". In: Sci Rep 6. Edition: 
2016/02/26, p. 21602. ISSN: 2045-2322 (Electronic) 2045-2322 (Linking). DOI: 10 . $1038 /$ srep21602. URL: https://www.ncbi.nlm.nih.gov/pubmed/26905010.

Locke, Adam E. et al. (Feb. 1, 2015). "Genetic studies of body mass index yield new insights for obesity biology". In: Nature 518.7538, pp. 197-206. ISSN: 1476-4687. DOI: 10 . 1038 / nature14177. URL: https://doi .org/10.1038/nature14177.

Lonardo, Enza et al. (Nov. 4, 2011). “Nodal/Activin Signaling Drives Self-Renewal and Tumorigenicity of Pancreatic Cancer Stem Cells and Provides a Target for Combined Drug Therapy". In: Cell Stem Cell 9.5, pp. 433-446. ISSN: 1934-5909. DOI: 10 . 1016 / j . stem. 2011.10.001. URL: https : / www. sciencedirect.com/science/article/pii/ S1934590911004814.

Longo, M. et al. (May 13, 2019). “Adipose Tissue Dysfunction as Determinant of ObesityAssociated Metabolic Complications". In: Int J Mol Sci 20.9. Edition: 2019/05/16. ISSN: 1422-0067 (Electronic) 1422-0067 (Linking). DOI: 10 . 3390 / ijms20092358. URL: https : //www.ncbi.nlm.nih.gov/pubmed/31085992.

Loos, R. J. and G. S. Yeo (Jan. 2014). "The bigger picture of FTO: the first GWAS-identified obesity gene". In: Nat Rev Endocrinol 10.1. Edition: 2013/11/20, pp. 51-61. ISSN: 1759-5029 (Print) 1759-5029. DOI: 10.1038/nrendo. 2013.227.

Lynes, M. D. and Y. H. Tseng (Jan. 2018). “Deciphering adipose tissue heterogeneity". In: Ann N Y Acad Sci 1411.1. Edition: 2017/08/02, pp. 5-20. ISSN: 0077-8923 (Print) 0077-8923. DOI: $10.1111 /$ nyas. 13398.

Magnusson, Björn et al. (May 7, 2010). "Activin B inhibits lipolysis in 3T3-L1 adipocytes". In: Biochemical and Biophysical Research Communications 395.3, pp. 373-376. ISSN: 0006-291X. DOI: $10.1016 / j$. bbrc. 2010.04 .022. URL: https : / www . sciencedirect.com/science/ article/pii/S0006291X10006844.

Marchesini, Giulio et al. (2008). “Obesity-Associated Liver Disease”. In: The Journal of Clinical Endocrinology \& Metabolism 93.11, s74-s80. ISSN: 0021-972X. DOI: 10.1210/jc . 2008-1399. URL: https://doi.org/10.1210/jc.2008-1399 (visited on 06/19/2021).

Massagué, J. (1998). “TGF-beta signal transduction”. In: Annu Rev Biochem 67. Edition: 1998/10/06, pp. 753-91. ISSN: 0066-4154 (Print) 0066-4154. DOI: 10 . 1146 / annurev . biochem.67.1.753.

Massagué, Joan (Dec. 1, 2000). "How cells read TGF- $\beta$ signals". In: Nature Reviews Molecular Cell Biology 1.3, pp. 169-178. ISSN: 1471-0080. DOI: 10 . 1038 / 35043051. URL: https : //doi.org/10.1038/35043051.

Matta, J. et al. (May 2018). "[French and worldwide epidemiology of obesity]". In: Presse Med 47.5. Edition: 2018/04/29, pp. 434-438. ISSN: 0755-4982. DOI: 10.1016/j . Ipm. 2018. 03.023.

Matzuk, M. M. (May 1995). “Functional analysis of mammalian members of the transforming growth factor-beta superfamily". In: Trends Endocrinol Metab 6.4. Edition: 1995/05/01, pp. 120-7. ISSN: 1043-2760 (Print) 1043-2760 (Linking). DOI: 10 . 1016/1043-2760 (95) 00032-d. URL: https://www.ncbi.nlm.nih.gov/pubmed/18406693. 
Merkestein, Myrte et al. (Apr. 17, 2015). "FTO influences adipogenesis by regulating mitotic clonal expansion". In: Nature Communications 6.1, p. 6792. ISSN: 2041-1723. DOI: 10.1038/ ncomms7792. URL: https://doi.org/10.1038/ncomms7792.

Merrick, D. et al. (Apr. 26, 2019). "Identification of a mesenchymal progenitor cell hierarchy in adipose tissue". In: Science 364.6438. Edition: 2019/04/27. ISSN: 0036-8075 (Print) 0036-8075. DOI: $10.1126 /$ science . aav2501.

Mittal, B. (May 2019). "Subcutaneous adipose tissue \& visceral adipose tissue". In: Indian J Med Res 149.5. Edition: 2019/08/17, pp. 571-573. ISSN: 0971-5916 (Print) 0971-5916. DOI: 10.4103/ijmr.IJMR_1910_18.

Miyoshi, H. et al. (June 9, 2006). "Perilipin promotes hormone-sensitive lipase-mediated adipocyte lipolysis via phosphorylation-dependent and -independent mechanisms". In: J Biol Chem 281.23. Edition: 2006/04/06, pp. 15837-44. ISSN: 0021-9258 (Print) 0021-9258. DOI: $10.1074 / \mathrm{jbc} . \mathrm{M601097200.}$

Morikawa, M., R. Derynck, and K. Miyazono (May 2, 2016). "TGF- $\beta$ and the TGF- $\beta$ Family: Context-Dependent Roles in Cell and Tissue Physiology". In: Cold Spring Harb Perspect Biol 8.5. Edition: 2016/05/04. ISSN: 1943-0264. DOI: 10.1101/cshperspect . a021873.

Moustakas, A., S. Souchelnytskyi, and C. H. Heldin (Dec. 2001). "Smad regulation in TGFbeta signal transduction". In: J Cell Sci 114 (Pt 24). Edition: 2002/01/17, pp. 4359-69. ISSN: 0021-9533 (Print) 0021-9533.

Mullen, A. C. et al. (Oct. 28, 2011). "Master transcription factors determine cell-type-specific responses to TGF- $\beta$ signaling". In: Cell 147.3. Edition: 2011/11/01, pp. 565-76. ISSN: 0092-8674 (Print) 0092-8674. DOI: 10.1016/j . cell.2011.08.050.

Munekata, K. and K. Sakamoto (Dec. 2009). "Forkhead transcription factor Foxo1 is essential for adipocyte differentiation". In: In Vitro Cell Dev Biol Anim 45.10. Edition: 2009/07/09, pp. 642-51. ISSN: 1071-2690. DOI: 10.1007/s11626-009-9230-5.

Murphy, E. Angela, Kandy T. Velazquez, and Kyle M. Herbert (2015). "Influence of high-fat diet on gut microbiota: a driving force for chronic disease risk". In: Curr Opin Clin Nutr Metab Care 18.5, pp. 515-520. ISSN: 1473-6519 1363-1950. DOI: 10 . 1097 / MCO . 0000000000000209. PMID: 26154278. URL: https : / / pubmed . ncbi . nlm . nih . gov / 26154278\%20https://www.ncbi.nlm.nih.gov/pmc/articles/PMC4578152/.

Nakae, J. et al. (Jan. 2003). "The forkhead transcription factor Foxo1 regulates adipocyte differentiation". In: Dev Cell 4.1. Edition: 2003/01/18, pp. 119-29. ISSN: 1534-5807 (Print) 1534-5807. DOI: $10.1016 / \mathrm{s} 1534-5807$ (02)00401-x.

Namwanje, M. and C. W. Brown (July 1, 2016). "Activins and Inhibins: Roles in Development, Physiology, and Disease". In: Cold Spring Harb Perspect Biol 8.7. Edition: 2016/06/23. ISSN: 1943-0264 (Electronic) 1943-0264 (Linking). DOI: 10 . 1101/cshperspect . a021881. URL: https://www.ncbi.nlm.nih.gov/pubmed/27328872.

Niazi, Robina Khan et al. (Sept. 5, 2019). "Screening of 31 genes involved in monogenic forms of obesity in 23 Pakistani probands with early-onset childhood obesity: a case report". In: BMC Medical Genetics 20.1, p. 152. ISSN: 1471-2350. DOI: 10.1186/s12881-019-0886-8. URL: https://doi .org/10.1186/s12881-019-0886-8. 
Nomura, M. and E. Li (June 25, 1998). "Smad2 role in mesoderm formation, left-right patterning and craniofacial development". In: Nature 393.6687. Edition: 1998/07/09, pp. 786-90. ISSN: 0028-0836 (Print) 0028-0836 (Linking). DOI: 10 . 1038/31693. URL: https : //www.ncbi.nlm.nih.gov/pubmed/9655392.

Nomura, M., H. L. Zhu, et al. (Jan. 2014). "SMAD2 disruption in mouse pancreatic beta cells leads to islet hyperplasia and impaired insulin secretion due to the attenuation of ATP-sensitive K+ channel activity". In: Diabetologia 57.1. Edition: 2013/09/27, pp. 157-66. ISSN: 0012-186x. DOI: 10.1007/s00125-013-3062-2.

O'Keefe, S. J. (Dec. 2016). "Diet, microorganisms and their metabolites, and colon cancer". In: Nat Rev Gastroenterol Hepatol 13.12. Edition: 2016/11/17, pp. 691-706. ISSN: 1759-5045 (Print) 1759-5045. DOI: 10.1038/nrgastro.2016.165.

Ober, Carole, Dagan A. Loisel, and Yoav Gilad (Dec. 1, 2008). "Sex-specific genetic architecture of human disease". In: Nature Reviews Genetics 9.12, pp. 911-922. ISSN: 1471-0064. DOI: $10.1038 / \mathrm{nrg} 2415$. URL: https ://doi .org/10.1038/nrg2415.

Olsen, Oddrun Elise et al. (2020). "Activins as Dual Specificity TGF- $\beta$ Family Molecules: SMAD-Activation via Activin- and BMP-Type 1 Receptors". In: Biomolecules 10.4, p. 519. ISSN: 2218-273X. URL: https : //www . mdpi . com/2218-273X/10/4/519.

Paik, J. et al. (2020). "Validation studies for germ-free Smad3(-/-) mice as a bio-assay to test the causative role of fecal microbiomes in IBD". In: Gut Microbes 11.1. Edition: 2019/05/30, pp. 21-31. ISSN: 1949-0976 (Print) 1949-0976. DOI: 10 .1080/19490976 . 2019.1611151.

Poniatowski $Ł$, A. et al. (2015). "Transforming growth factor Beta family: insight into the role of growth factors in regulation of fracture healing biology and potential clinical applications". In: Mediators Inflamm 2015. Edition: 2015/02/25, p. 137823. ISSN: 0962-9351 (Print) 0962-9351. DOI: 10.1155/2015/137823.

Pyrina, I. et al. (2020). "Fate of Adipose Progenitor Cells in Obesity-Related Chronic Inflammation". In: Front Cell Dev Biol 8. Edition: 2020/08/08, p. 644. ISSN: 2296-634X (Print) 2296-634x. DOI: 10.3389/f cell.2020.00644.

Rabe, K. et al. (Nov. 2008). "Adipokines and insulin resistance". In: Mol Med 14.11. Edition: 2008/11/15, pp. 741-51. ISSN: 1528-3658 (Electronic) 1076-1551 (Linking). DOI: 10 . 2119/ 2008-00058. Rabe. URL: https://www.ncbi.nlm.nih.gov/pubmed/19009016.

Ridaura, Vanessa K. et al. (2013). "Gut microbiota from twins discordant for obesity modulate metabolism in mice". In: Science 341.6150, pp. 1241214-1241214. ISSN: 1095-9203 0036-8075. DOI: 10 . 1126/science. 1241214. PMID: 24009397. URL: https : / pubmed . ncbi .nlm . nih . gov/24009397\%20https : / /www . ncbi .nlm . nih . gov/pmc/articles / PMC3829625/.

Ronquillo, M. D. et al. (May 2019). “Different gene expression profiles in subcutaneous \& visceral adipose tissues from Mexican patients with obesity". In: Indian J Med Res 149.5. Edition: 2019/08/17, pp. 616-626. ISSN: 0971-5916 (Print) 0971-5916. DOI: $10.4103 / \mathrm{ijmr}$. IJMR_1165_17.

Rosen, E. D., C. H. Hsu, et al. (Jan. 1, 2002). “C/EBPalpha induces adipogenesis through PPARgamma: a unified pathway". In: Genes Dev 16.1. Edition: 2002/01/10, pp. 22-6. ISSN: 0890-9369 (Print) 0890-9369. DOI: 10.1101/gad . 948702. 
Rosen, E. D. and B. M. Spiegelman (Dec. 14, 2006). “Adipocytes as regulators of energy balance and glucose homeostasis". In: Nature 444.7121. Edition: 2006/12/15, pp. 847-53. ISSN: 0028-0836 (Print) 0028-0836. DOI: 10.1038/nature05483.

Rosen, Evan D. and Ormond A. MacDougald (Dec. 1, 2006). "Adipocyte differentiation from the inside out". In: Nature Reviews Molecular Cell Biology 7.12, pp. 885-896. ISSN: 1471-0080. DOI: $10.1038 / \mathrm{nrm} 2066$. URL: https://doi .org/10.1038/nrm2066.

Rosenwald, Matthias and Christian Wolfrum (2014). "The origin and definition of brite versus white and classical brown adipocytes". In: Adipocyte 3.1. Edition: 2013/08/28, pp. 4-9. ISSN: 2162-3945 2162-397X. DOI: 10.4161 /adip. 26232. PMID: 24575363. URL: https : //pubmed.ncbi.nlm.nih.gov/24575363\%20https://www .ncbi.nlm.nih.gov/ pmc/articles/PMC3917931/.

Rutkowski, J. M., J. H. Stern, and P. E. Scherer (Mar. 2, 2015). “The cell biology of fat expansion". In: J Cell Biol 208.5. Edition: 2015/03/04, pp. 501-12. ISSN: 1540-8140 (Electronic) 0021-9525 (Linking). DOI: 10 .1083/jcb. 201409063. URL: https : / www .ncbi.nlm.nih . gov/pubmed/25733711.

Samaras, K. et al. (May 2010). "Subcutaneous and visceral adipose tissue gene expression of serum adipokines that predict type 2 diabetes". In: Obesity (Silver Spring) 18.5. Edition: 2009/12/19, pp. 884-9. ISSN: 1930-7381. DOI: 10.1038/oby. 2009. 443.

Sanyaolu, Adekunle et al. (2019). "Childhood and Adolescent Obesity in the United States: A Public Health Concern". In: Glob Pediatr Health 6, pp. 2333794X198913052333794X19891305. ISSN: 2333-794X. DOI: 10.1177/2333794X19891305. PMID: 31832491. URL: https : //pubmed.ncbi .nlm.nih.gov/31832491\%20https : //www . ncbi.nlm.nih . gov/pmc/articles/PMC6887808/.

Sarjeant, K. and J. M. Stephens (Sept. 1, 2012). "Adipogenesis". In: Cold Spring Harb Perspect Biol 4.9. Edition: 2012/09/07, a008417. ISSN: 1943-0264 (Electronic) 1943-0264 (Linking). DOI: 10 .1101/cshperspect . a008417. URL: https : / www .ncbi .nlm.nih.gov/pubmed/ 22952395.

Schrewe, H. et al. (July 1994). "Mice homozygous for a null mutation of activin beta B are viable and fertile". In: Mech Dev 47.1. Edition: 1994/07/01, pp. 43-51. ISSN: 09254773 (Print) 0925-4773 (Linking). DOI: 10 . 1016/0925 - 4773 (94) 90094 - 9. URL: https : //www.ncbi.nlm.nih.gov/pubmed/7947320.

Seoane, J. et al. (Apr. 16, 2004). "Integration of Smad and forkhead pathways in the control of neuroepithelial and glioblastoma cell proliferation". In: Cell 117.2. Edition: 2004/04/16, pp. 211-23. ISSN: 0092-8674 (Print) 0092-8674. DOI: 10 .1016/s0092-8674 (04) 00298-3.

Shao, M. et al. (Jan. 2017). "Fetal development of subcutaneous white adipose tissue is dependent on Zfp423". In: Mol Metab 6.1. Edition: 2017/01/27, pp. 111-124. ISSN: 22128778 (Electronic) 2212-8778 (Linking). DOI: 10 . $1016 / \mathrm{j}$. molmet . 2016 . 11 . 009. URL: https://www.ncbi.nlm.nih.gov/pubmed/28123942.

Shen, J. J. et al. (Jan. 2009). "Deficiency of growth differentiation factor 3 protects against dietinduced obesity by selectively acting on white adipose". In: Mol Endocrinol 23.1. Edition: 2008/11/15, pp. 113-23. ISSN: 0888-8809 (Print) 0888-8809. DOI: 10.1210/me . 2007-0322. 
Shen, X. et al. (Dec. 1998). "TGF-beta-induced phosphorylation of Smad3 regulates its interaction with coactivator p300/CREB-binding protein". In: Mol Biol Cell 9.12. Edition: 1998/12/08, pp. 3309-19. ISSN: 1059-1524 (Print) 1059-1524. DOI: 10 . 1091 /mbc . 9 . 12 . 3309.

Shi, Y. and J. Massagué (June 13, 2003). "Mechanisms of TGF-beta signaling from cell membrane to the nucleus". In: Cell 113.6. Edition: 2003/06/18, pp. 685-700. ISSN: 00928674 (Print) 0092-8674. DOI: 10.1016/s0092-8674 (03) 00432-x.

Shi, Yigong et al. (Sept. 4, 1998). “Crystal Structure of a Smad MH1 Domain Bound to DNA: Insights on DNA Binding in TGF- $\beta$ Signaling". In: Cell 94.5, pp. 585-594. ISSN: 0092-8674. DOI: 10 .1016/S0092-8674(00)81600-1. URL: https : / www . sciencedirect. $\mathrm{com} / \mathrm{science/article/pii/S0092867400816001.}$

Sjöholm, Kajsa et al. (June 16, 2006). "The expression of inhibin beta B is high in human adipocytes, reduced by weight loss, and correlates to factors implicated in metabolic disease". In: Biochemical and Biophysical Research Communications 344.4, pp. 1308-1314. ISSN: 0006-291X. DOI: 10.1016/j . bbrc . 2006.04.030. URL: https : //www . sciencedirect. $\mathrm{com} / \mathrm{science/article/pii/S0006291X06008606.}$

Song, W. et al. (Aug. 8, 2017). "Activin signaling mediates muscle-to-adipose communication in a mitochondria dysfunction-associated obesity model". In: Proceedings of the National Academy of Sciences of the United States of America 114.32. Edition: 2017/07/26, pp. 85968601. ISSN: 0027-8424 (Print) 0027-8424. DOI: 10.1073/pnas. 1708037114.

Stanford, K. I. and L. J. Goodyear (Oct. 2013). "The therapeutic potential of brown adipose tissue". In: Hepatobiliary Surg Nutr 2.5. Edition: 2014/02/27, pp. 286-7. ISSN: 2304-3881 (Print) 2304-3881. DOI: 10.3978/j . issn. 2304-3881.2013.09.02.

Stern, Jennifer H., Joseph M. Rutkowski, and Philipp E. Scherer (2016). “Adiponectin, Leptin, and Fatty Acids in the Maintenance of Metabolic Homeostasis through Adipose Tissue Crosstalk". In: Cell Metab 23.5, pp. 770-784. ISSN: 1932-7420 1550-4131. DOI: 10.1016 / j . cmet . 2016 .04 .011. PMID: 27166942. URL: https : / pubmed . ncbi .nlm.nih . gov/ $27166942 \% 20$ https://www.ncbi.nlm.nih.gov/pmc/articles/PMC4864949/.

Sul, H. S. et al. (Nov. 1, 2000). "Function of pref-1 as an inhibitor of adipocyte differentiation". In: International Journal of Obesity 24.4, S15-S19. ISSN: 1476-5497. DOI: 10.1038 / s j . i jo . 0801494. URL: https://doi.org/10.1038/sj.ijo.0801494.

Pi-Sunyer, X. (Nov. 2009). "The medical risks of obesity". In: Postgrad Med 121.6. Edition: 2009/11/27, pp. 21-33. ISSN: 0032-5481 (Print) 0032-5481. DOI: 10 . 3810/pgm . 2009 . 11. 2074.

Tan, C. K., H. C. Chong, et al. (Mar. 5, 2012). “Getting 'Smad' about obesity and diabetes". In: Nutr Diabetes 2. Edition: 2012/01/01, e29. ISSN: 2044-4052 (Electronic) 2044-4052 (Linking). DOI: 10.1038/nutd.2012.1. URL: https://www.ncbi.nlm.nih.gov/pubmed/23449528.

Tan, C. K., N. Leuenberger, et al. (Feb. 2011). "Smad3 deficiency in mice protects against insulin resistance and obesity induced by a high-fat diet". In: Diabetes 60.2. Edition: 2011/01/29, pp. 464-76. ISSN: 1939-327X (Electronic) 0012-1797 (Linking). DOI: 10 . 2337 / db10-0801. URL: https://www.ncbi.nlm.nih.gov/pubmed/21270259. 
Tan, C. K., E. H. Tan, et al. (June 19, 2013). "SMAD3 deficiency promotes inflammatory aortic aneurysms in angiotensin II-infused mice via activation of iNOS". In: J Am Heart Assoc 2.3. Edition: 2013/06/21, e000269. ISSN: 2047-9980 (Electronic) 2047-9980 (Linking). DOI: 10.1161/JAHA.113.000269. URL: https://www.ncbi.nlm.nih.gov/pubmed/23782924.

Tang, Q. Q., T. C. Otto, and M. D. Lane (June 29, 2004). “Commitment of C3H10T1/2 pluripotent stem cells to the adipocyte lineage". In: Proc Natl Acad Sci U S A 101.26. Edition: 2004/06/24, pp. 9607-11. ISSN: 0027-8424 (Print) 0027-8424. DOI: 10.1073/pnas . 0403100101.

Tao, J. J. et al. (Sept. 15, 2019). "First-in-Human Phase I Study of the Activin A Inhibitor, STM 434, in Patients with Granulosa Cell Ovarian Cancer and Other Advanced Solid Tumors". In: Clin Cancer Res 25.18. Edition: 2019/05/10, pp. 5458-5465. ISSN: 1078-0432 (Print) 1078-0432 (Linking). DOI: 10 . $1158 / 1078$ - 0432 . CCR - $19-1065$. URL: https : //www.ncbi.nlm.nih.gov/pubmed/31068369.

Thursby, E. and N. Juge (May 16, 2017). "Introduction to the human gut microbiota". In: Biochem J 474.11. Edition: 2017/05/18, pp. 1823-1836. ISSN: 0264-6021 (Print) 0264-6021. DOI: $10.1042 / \mathrm{bcj} 20160510$.

Tiano, J. P., D. A. Springer, and S. G. Rane (Mar. 20, 2015). “SMAD3 negatively regulates serum irisin and skeletal muscle FNDC5 and peroxisome proliferator-activated receptor $\gamma$ coactivator 1- $\alpha$ (PGC-1 $\alpha$ ) during exercise". In: J Biol Chem 290.12. Edition: 2015/02/05, pp. 7671-84. ISSN: 0021-9258 (Print) 0021-9258. DOI: 10.1074/jbc. M114.617399.

Tran, Thien T. et al. (May 7, 2008). "Beneficial Effects of Subcutaneous Fat Transplantation on Metabolism”. In: Cell Metabolism 7.5, pp. 410-420. ISSN: 1550-4131. DOI: 10.1016/j . cmet . 2008.04 .004. URL: https : / /www . sciencedirect . com/science/article/pii/ S1550413108001149.

Traustadottir, G. A. et al. (Oct. 1, 2013). "Preadipocytes proliferate and differentiate under the guidance of Delta-like 1 homolog (DLK1)". In: Adipocyte 2.4. Edition: 2013/09/21, pp. 272-5. ISSN: 2162-3945 (Print) 2162-3945 (Linking). DOI: 10 . 4161/adip . 24994. URL: https://www.ncbi.nlm.nih.gov/pubmed/24052905.

Tseng, Yu-Hua et al. (2008). "New role of bone morphogenetic protein 7 in brown adipogenesis and energy expenditure". In: Nature 454.7207, pp. 1000-1004. ISSN: 1476-4687 0028-0836. DOI: 10.1038/nature07221. PMID: 18719589. URL: https://pubmed.ncbi.nlm.nih.gov/ 18719589\%20https://www.ncbi.nlm.nih.gov/pmc/articles/PMC2745972/.

Tsuchida, K. et al. (May 31, 2004). "Activin isoforms signal through type I receptor serine/threonine kinase ALK7". In: Mol Cell Endocrinol 220.1. Edition: 2004/06/16, pp. 59-65. ISSN: 0303-7207 (Print) 0303-7207. DOI: 10.1016/j . mce. 2004.03.009.

Tsuchida, Kunihiro et al. (June 18, 2009). “Activin signaling as an emerging target for therapeutic interventions". In: Cell Communication and Signaling 7.1, p. 15. ISSN: 1478-811X. DOI: 10.1186/1478-811X-7-15. URL: https://doi .org/10.1186/1478-811X-7-15.

Tsurutani, Y. et al. (Apr. 1, 2011). "The roles of transforming growth factor-beta and Smad3 signaling in adipocyte differentiation and obesity". In: Biochem Biophys Res Commun 407.1. Edition: 2011/03/02, pp. 68-73. ISSN: 1090-2104 (Electronic) 0006-291X (Linking). DOI: 10 . 1016/j. bbrc. 2011.02.106. URL: https://www.ncbi.nlm.nih.gov/pubmed/21356196. 
Turnbaugh, P. J., M. Hamady, et al. (Jan. 22, 2009). “A core gut microbiome in obese and lean twins". In: Nature 457.7228. Edition: 2008/12/02, pp. 480-4. ISSN: 0028-0836 (Print) 0028-0836. DOI: $10.1038 /$ nature07540.

Turnbaugh, P. J., R. E. Ley, et al. (Dec. 21, 2006). "An obesity-associated gut microbiome with increased capacity for energy harvest". In: Nature 444.7122. Edition: 2006/12/22, pp. 1027-31. ISSN: 0028-0836. DOI: 10.1038/nature05414.

Turnbaugh, P. J., V. K. Ridaura, et al. (Nov. 11, 2009). “The effect of diet on the human gut microbiome: a metagenomic analysis in humanized gnotobiotic mice". In: Sci Transl Med 1.6. Edition: 2010/04/07, 6ra14. ISSN: 1946-6234 (Print) 1946-6234. DOI: 10.1126 / scitranslmed. 3000322.

Unger, R. H. et al. (Mar. 2010). "Lipid homeostasis, lipotoxicity and the metabolic syndrome". In: Biochim Biophys Acta 1801.3. Edition: 2009/12/02, pp. 209-14. ISSN: 0006-3002 (Print) 0006-3002 (Linking). DOI: 10 . 1016/ j . bbalip . 2009 . 10 . 006. URL: https : / www . ncbi . nlm.nih.gov/pubmed/19948243.

Vassalli, A. et al. (Feb. 15, 1994). "Activin/inhibin beta B subunit gene disruption leads to defects in eyelid development and female reproduction". In: Genes Dev 8.4. Edition: 1994/02/15, pp. 414-27. ISSN: 0890-9369 (Print) 0890-9369 (Linking). DOI: 10 .1101/gad . 8. 4.414. URL: https://www.ncbi.nlm.nih.gov/pubmed/8125256.

Waldén, T. B. et al. (Jan. 1, 2012). "Recruited vs. nonrecruited molecular signatures of brown, "brite," and white adipose tissues". In: Am J Physiol Endocrinol Metab 302.1. Edition: 2011/08/11, E19-31. ISSN: 0193-1849. DOI: 10.1152/ajpendo.00249.2011.

Waldrip, W. R. et al. (Mar. 20, 1998). "Smad2 signaling in extraembryonic tissues determines anterior-posterior polarity of the early mouse embryo". In: Cell 92.6. Edition: 1998/04/07, pp. 797-808. ISSN: 0092-8674 (Print) 0092-8674 (Linking). DOI: 10.1016/s0092-8674 (00) 81407-5. URL: https://www.ncbi.nlm.nih.gov/pubmed/9529255.

Wang, D. et al. (Jan. 2020). "LSD1 mediates microbial metabolite butyrate-induced thermogenesis in brown and white adipose tissue". In: Metabolism 102. Edition: 2019/11/18, p. 154011. ISSN: 0026-0495. DOI: 10.1016/j. metabol.2019.154011.

Wang, L. et al. (Sept. 26, 2018). "FoxG1 facilitates proliferation and inhibits differentiation by downregulating FoxO/Smad signaling in glioblastoma". In: Biochem Biophys Res Commun 504.1. Edition: 2018/09/03, pp. 46-53. ISSN: 0006-291x. DOI: 10.1016/j . bbrc. 2018.08. 118.

Wang, P. et al. (July 2008). "The secretory function of adipocytes in the physiology of white adipose tissue". In: J Cell Physiol 216.1. Edition: 2008/02/12, pp. 3-13. ISSN: 1097-4652 (Electronic) 0021-9541 (Linking). DOI: 10.1002/jcp.21386. URL: https ://www.ncbi.nlm. nih.gov/pubmed/18264975.

Wang, Ya-Wen et al. (2017). "Physiological and metabolic differences between visceral and subcutaneous adipose tissues in Nile tilapia (Oreochromis niloticus)". In: American Journal of Physiology-Regulatory, Integrative and Comparative Physiology 313.5, R608-R619. DOI: 10 . 1152/ajpregu . 00071 . 2017. URL: https : / / journals . physiology . org/doi / abs/10.1152/ajpregu.00071.2017. 
Wang, Yuhui, Kyung-Ah Kim, et al. (2006). "Pref-1, a Preadipocyte Secreted Factor That Inhibits Adipogenesis". In: The Journal of Nutrition 136.12, pp. 2953-2956. ISSN: 0022-3166. DOI: 10.1093/jn/136.12.2953. URL: https : / / doi .org/10.1093/jn/136 .12.2953 (visited on 07/06/2021).

Wang, Yuhui and Hei Sook Sul (Mar. 4, 2009). "Pref-1 Regulates Mesenchymal Cell Commitment and Differentiation through Sox9". In: Cell Metabolism 9.3, pp. 287-302. ISSN: 1550-4131. DOI: 10.1016/j . cmet.2009.01 .013. URL: https://www. sciencedirect.com/ science/article/pii/S1550413109000382.

Wankhade, U. D. et al. (Oct. 2018). "TGF-beta receptor 1 regulates progenitors that promote browning of white fat". In: Mol Metab 16. Edition: 2018/08/14, pp. 160-171. ISSN: 22128778 (Electronic) 2212-8778 (Linking). DOI: 10 . 1016/ j . molmet . 2018 . 07 . 008. URL: https://www.ncbi.nlm.nih.gov/pubmed/30100246.

Weinstein, M. et al. (Aug. 4, 1998). "Failure of egg cylinder elongation and mesoderm induction in mouse embryos lacking the tumor suppressor smad2". In: Proc Natl Acad Sci U S A 95.16. Edition: 1998/08/05, pp. 9378-83. ISSN: 0027-8424 (Print) 0027-8424 (Linking). DOI: 10 . 1073 / pnas . 95 . 16 .9378. URL: https : / / www . ncbi .nlm . nih . gov/pubmed / 9689088.

Weir, C. B. and A. Jan (2021). "BMI Classification Percentile And Cut Off Points". In: StatPearls. Treasure Island (FL): StatPearls Publishing Copyright (C) 2021, StatPearls Publishing LLC.

Whitlock, E. et al. (Sept. 2008). "Effectiveness of weight management programs in children and adolescents". In: Evid Rep Technol Assess (Full Rep) 170. Edition: 2009/05/05, pp. 1-308. ISSN: 1530-4396 (Print) 1530-4396.

Wright, S. M. and L. J. Aronne (Oct. 2012). "Causes of obesity". In: Abdom Imaging 37.5. Edition: 2012/03/20, pp. 730-2. ISSN: 0942-8925. DOI: 10.1007/s00261-012-9862-x.

$\mathrm{Wu}$, J. et al. (July 20, 2012). "Beige adipocytes are a distinct type of thermogenic fat cell in mouse and human". In: Cell 150.2. Edition: 2012/07/17, pp. 366-76. ISSN: 0092-8674 (Print) 0092-8674. DOI: 10.1016/j . cell.2012.05.016.

Xiao, Han and Sona Kang (2020). "The Role of the Gut Microbiome in Energy Balance With a Focus on the Gut-Adipose Tissue Axis". In: Front Genet 11, pp. 297-297. ISSN: 1664-8021. DOI: 10 . 3389/fgene. 2020 . 00297. PMID: 32318095. URL: https : / pubmed . ncbi .nlm . nih.gov/32318095\%20https://www.ncbi.nlm.nih.gov/pmc/articles/PMC7154186/.

Xiao, Z. et al. (Sept. 5, 2003). "A novel nuclear export signal in Smad1 is essential for its signaling activity". In: J Biol Chem 278.36. Edition: 2003/06/25, pp. 34245-52. ISSN: 0021-9258 (Print) 0021-9258. DOI: 10.1074/jbc.M301596200.

$\mathrm{Xu}, \mathrm{W}$. et al. (May 23, 2000). "Ski acts as a co-repressor with Smad2 and Smad3 to regulate the response to type beta transforming growth factor". In: Proc Natl Acad Sci U S A 97.11. Edition: 2000/05/17, pp. 5924-9. ISSN: 0027-8424 (Print) 0027-8424. DOI: 10.1073/pnas . 090097797.

Xu, X. et al. (Dec. 3, 2013). "Obesity activates a program of lysosomal-dependent lipid metabolism in adipose tissue macrophages independently of classic activation". In: Cell 
Metab 18.6. Edition: 2013/12/10, pp. 816-30. ISSN: 1550-4131 (Print) 1550-4131. DOI: 10.1016/j.cmet.2013.11.001.

Xue, L. X. et al. (Nov. 18, 2016). “Activin A/Smads signaling pathway negatively regulates Oxygen Glucose Deprivation-induced autophagy via suppression of JNK and p38 MAPK pathways in neuronal PC12 cells". In: Biochem Biophys Res Commun 480.3. Edition: 2016/10/27, pp. 355-361. ISSN: 1090-2104 (Electronic) 0006-291X (Linking). DOI: 10 . 1016/ j.bbrc. 2016.10.050. URL: https://www.ncbi.nlm.nih.gov/pubmed/27769861.

Yadav, H., S. Devalaraja, et al. (Feb. 24, 2017). "TGF-beta1/Smad3 Pathway Targets PP2AAMPK-FoxO1 Signaling to Regulate Hepatic Gluconeogenesis". In: J Biol Chem 292.8. Edition: 2017/01/11, pp. 3420-3432. ISSN: 1083-351X (Electronic) 0021-9258 (Linking). DOI: 10.1074/jbc.M116.764910. URL: https://www.ncbi.nlm.nih.gov/pubmed/28069811.

Yadav, H., C. Quijano, et al. (July 6, 2011). "Protection from obesity and diabetes by blockade of TGF-beta/Smad3 signaling". In: Cell Metab 14.1. Edition: 2011/07/05, pp. 67-79. ISSN: 1932-7420 (Electronic) 1550-4131 (Linking). DOI: 10 . 1016/ j . cmet . 2011 . 04 . 013. URL: https://www.ncbi.nlm.nih.gov/pubmed/21723505.

Yadav, H. and S. G. Rane (2012). "TGF-beta/Smad3 Signaling Regulates Brown Adipocyte Induction in White Adipose Tissue". In: Front Endocrinol (Lausanne) 3. Edition: 2012/06/02, p. 35. ISSN: 1664-2392 (Electronic) 1664-2392 (Linking). DOI: 10.3389/f endo . 2012 . 00035. URL: https://www.ncbi.nlm.nih.gov/pubmed/22654861.

Yang, X. et al. (Mar. 1, 1999). "Targeted disruption of SMAD3 results in impaired mucosal immunity and diminished T cell responsiveness to TGF-beta". In: EMBO J 18.5. Edition: 1999/03/04, pp. 1280-91. ISSN: 0261-4189 (Print) 0261-4189 (Linking). DOI: 10 . 1093 / emboj/18.5.1280. URL: https://www.ncbi.nlm.nih.gov/pubmed/10064594.

Zamani, Nader and Chester W. Brown (2011). "Emerging Roles for the Transforming Growth Factor- $\beta$ Superfamily in Regulating Adiposity and Energy Expenditure". In: Endocrine Reviews 32.3, pp. 387-403. ISSN: 0163-769X. DOI: 10.1210/er .2010-0018. URL: https://doi.org/10.1210/er.2010-0018 (visited on 07/05/2021).

Zaragosi, L. E. et al. (Oct. 2010). “Activin a plays a critical role in proliferation and differentiation of human adipose progenitors". In: Diabetes 59.10. Edition: 2010/06/10, pp. 251321. ISSN: 1939-327X (Electronic) 0012-1797 (Linking). DOI: 10 . 2337 / db10 - 0013. URL: https://www.ncbi.nlm.nih.gov/pubmed/20530742.

Zhang, C. et al. (Oct. 2012). "Structural resilience of the gut microbiota in adult mice under high-fat dietary perturbations". In: Isme j 6.10. Edition: 2012/04/13, pp. 1848-57. ISSN: 1751-7362 (Print) 1751-7362. DOI: 10.1038/ismej . 2012 . 27.

Zhang, L. et al. (Feb. 2015). "Smad2 protects against TGF-beta1/Smad3-mediated collagen synthesis in human hepatic stellate cells during hepatic fibrosis". In: Mol Cell Biochem 400.1. Edition: 2014/10/30, pp. 17-28. ISSN: 1573-4919 (Electronic) 0300-8177 (Linking). DOI: 10 . 1007 /s11010-014-2258-1. URL: https : / /www .ncbi .nlm .nih . gov/pubmed/ 25351340.

Zhu, Q. et al. (Sept. 7, 2018). "The regulatory protein SnoN antagonizes activin/Smad2 protein signaling and thereby promotes adipocyte differentiation and obesity in mice". In: J Biol Chem 293.36. Edition: 2018/07/22, pp. 14100-14111. ISSN: 1083-351X (Electronic) 
0021-9258 (Linking). DOI: 10 . 1074/ jbc . RA118 . 003678. URL: https : //www . ncbi . nlm . nih.gov/pubmed/30030373.

Zhu, Y. et al. (Sept. 18, 1998). "Smad3 mutant mice develop metastatic colorectal cancer". In: Cell 94.6. Edition: 1998/09/30, pp. 703-14. ISSN: 0092-8674 (Print) 0092-8674 (Linking). DOI: 10.1016/s0092-8674(00)81730-4. URL: https://www.ncbi.nlm.nih.gov/pubmed/ 9753318.

Zhu, Y. P. et al. (Mar. 2017). "Smad3 mutant mice develop colon cancer with overexpression of COX-2". In: Oncol Lett 13.3. Edition: 2017/04/30, pp. 1535-1538. ISSN: 1792-1074 (Print) 1792-1074 (Linking). DOI: 10 . 3892/ol . 2017 . 5639. URL: https : //www . ncbi . nlm . nih . gov/pubmed/28454287.

Zi, Zhike, Douglas A. Chapnick, and Xuedong Liu (July 4, 2012). "Dynamics of TGF$\beta /$ Smad signaling". In: FEBS Letters 586.14, pp. 1921-1928. ISSN: 0014-5793. DOI: 10.1016/ j.febslet.2012.03.063. URL: https://www . sciencedirect.com/science/article/ $\mathrm{pii} / \mathrm{S} 0014579312002736$.

Ziętak, Marika et al. (June 14, 2016). "Altered Microbiota Contributes to Reduced DietInduced Obesity upon Cold Exposure". In: Cell Metabolism 23.6, pp. 1216-1223. ISSN: 1550-4131. DOI: 10.1016/j.cmet.2016.05.001. URL: https://www. sciencedirect.com/ science/article/pii/S1550413116302194. 


\section{Vita}

Roshan Kumari was born (1984) in a village, Sakarpura, Bihar INDIA and grew up in a university campus in a small town. She obtained her Bachelor of Science (B.Sc.) in 2004 and Master of Science (M.Sc.) degree in 2006 from the reputed university Tilka Manjhi Bhagalpur University, Bhagalpur, Bihar, INDIA. She joined at University of Tennessee Health Science Center, Memphis, TN in 2017. Roshan performed dissertation research under Dr. Chester W. Brown, a highly regarded physician, and a professor in Pediatrics-genetics department. She was matriculated in Integrated Biomedical Science Program in 2018. She expects to receive her doctorate degree in November 2021. 